IBL -32795

DE92 040381

\title{
Molecular Genetic and Molecular Evolutionary Studies on the Bacteriochlorophyll Synthesis Genes of Rhodobacter capsulatus \\ by
}

Donald $H$, Burke-Aguero

(Ph. D. Thesis)

\author{
Structural Biology Division \\ Lawrence Berkeley Laboratory \\ 1 Cyclotron Road \\ Berkeley, California 94720
}

August, 1992

This work was supported by the Office of Basic Energy Science, Biological Energy Division of the Department of Energy under Contract No. DE-ACO30-76F000978, by National Inscitutes of Health grant $G M$ 30786, and by a graduate research fellowship from BristolMeyers Squibb. 
Molecular Genetic and Molecular Evolutionary Studies on the Bacteriochlorophyll Synthesis Genes of Bhodobacter capsulatus

by

\section{Donald H. Burke-Agüero}

\section{Abstract}

Rhodobacter capsulatus is a purple bacterium capable of either aerobic or photosynthetic growth. It has proven itself to be very useful in genetic studies of photosynthesis. Forty-four genes clustered together into a recently sequenced 46 kilobase region of the chromosome are required to establish photosynthetic ability in $R$. capsulatus. Of these, approximately twenty are involved in bacteriochlorophyll synthesis. I examine eight "bch" genes in this thesis. Most of these had been incorrectly described in previous studies, and so the major task has been to identify the number and function of these genes. Six of these genes code for the two ring reductases. These first convert the universal precursor of all reaction center pigments, protochlorophyllide (PChlide), into a chlorin (the immediate precursor to chlorophyll $a$ ) and then into a bacteriochlorin. Each reductase is shown to be made up of three subunits. PChlide reductase is coded by the genes $b c h N, b c h B$, and $b c h L$. Proteins with arnino acid sequences markedly similar to those of $b c h N$ and bchL have been shown in other organisms to be required for chlorophyll synthesis; hence, their designation as $c h l N$ and $c h l B$. I show here that a third. chloroplast-encoded gene of heretofore unknown function shares amino acid identities with $b \operatorname{ch} B$ and is probably the third subunit of the plant PChlide reductase. The bchA locus, which encodes the chlorin reductase, is found to be made up of three separate, translationally coupled genes, refered to as bchX. $b c h Y$, and bchZ. Amino acid acid similarities between $b c h X, b c h L$, and the nitrogenase reductase protein $n f H$ suggest that all three classes of proteins 
share certain three-dimensional structural features, including elements that are central to the enzymatic mechanism of nifH. A mechanism for PChlide and chlorin reductions is proposed based on these similarities. Finally, PChlide reductase and chlorin reductase are clearly derived from a common ancestor which was itself a three-subunit reductase. Several lines of analysis lead to the conclusion that the ancestor of both enzyme systems reduced PChlide twice to produce bacteriochlorophyll, offering the first strong evidence that bacteriochlorophyll preceded chlorophyll as the ancestral reaction center pigment. 
This thesis is dedicated to my first teachers--my parents, Donald E. and Alice $M$. H. Burke and my siblings, Fred, Nancy, and John Burke--and to my new family, Hannia and Daniel Burke-Agüero. 
Acknowledgements

It was my brother, John, always curious, who taught me, even in my earliest memories of him, that the world can be understood. Even before I began kindergarten, his curiosity had begun to burn in me. A hearty thanks goes out to all who have shown me the way through twenty-four years of formal education from kindergarten to the completion of this thesis!

I want to thank Sam Lipson and Yu Sheng Zhu, who did their best to teach me the basics of lab work when I arrived at Berkeley, and Jason Kahn, Peter Spielmann, Joe Monforte, and Suzanne Cheng, who likewise tried to fill me in on the essentials of nucleic acid chemistry. The work that went into this thesis was built around the heroic DNA sequencing work of the everenthusiatic Marie Alberti. If $I$ have learned anything about how to work with bacteria and their DNA, then I must offer thanks to Greg Armstrong, David Cook, and Ma Dzwokai (Zack), I am especially appreciative of Enrique Dalmasso and David Alexander for always knowing more about scuba diving and chasing after the elusive abalone than I, and of David O'Brien for his genius in the structural engineering of sand. The friendship of all of these people has been a blessing.

I am fortunate to have been able to do my thesis work under the direction of Professor John Hearst, whose insight and creativity helped me try to think about problems in a different light than has been applied to them before, and to look for the fundamental assumptions behind every line of thinking. At the same time, his friendship and support outside the lab has shown the importance he places on the people he works with. 
Michael Senturia's personal and professional intensity and his insistence on excellence were marvelously contagious during the five years I spent in the University Orchestra under his direction. I feel honored to have earned his friendship and respect. L'Chaim, Michael!

Not enough can be said in appreciation for my wife, Hannia, who left a promising career in Costa Rica, fought immigration for ten months to get into this country, learned English from square one, and has played both full-time student and full-time mother for these last two and a half years. All the while she has offered steadfast encouragement and helped me from sinking into the morass of warped priorities that can be occasioned by dissertation research.

I am appreciative of having had the opportunity to travel to several major conferences and to exchange ideas with the leaders of several fields. As a result, I can also express my thanks to Professors Doug Rees, Jim Howard, Carl Bauer, Robert Blankenship, C. Neal Hunter, Diana Stein, David Eisenberg, Beverly Pierson, Laurie Mets, and Norman Pace for insightful discussions, for their constructive criticisms, and for sharing the results of their research prior to pubiication. I also want extend thanks to Arend Sidow, who collaborated with me on the phylogenetic analysis at the end of the thesis. David Koh's precision synthesis of oligonucleotides made much of the work possible. The work presented here was supported in part by National Institutes of Health grant GM 30786, by the Office of Basic Energy Science, Biological Energy Division of the Department of Energy under contract DE-ACO3076F000978, and by a graduate research fellowship from Bristol-Meyers Squibb. 
Dedication

Acknowledgements

Contents

vii

List of Tables and Figures

Chapter 1: Who, What, Where, When, Why, and How............................... 1

The biological context........................................................................ 2

Bacterial photosynthesis................................................................... 8

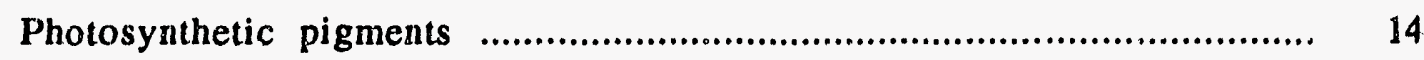

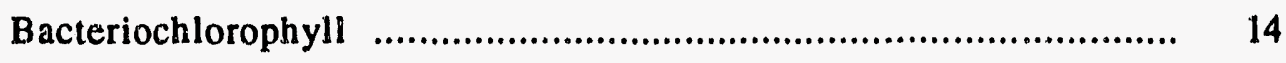

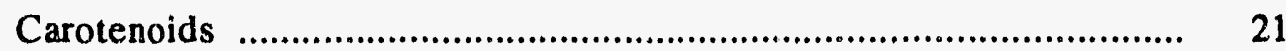

The Chromosomal Context ................................................................... 26

The photosynthesis gene cluster and organization of the thesis ....... 31

Chapter 2. Translational coupling in the $b c h C X Y Z$ operon of Rhodobacter capsulatus and the generality of chlorophyll $\mathrm{Fe}$ proteins in chlorophyll and bacteriochlorophyll synthesis ................. 36

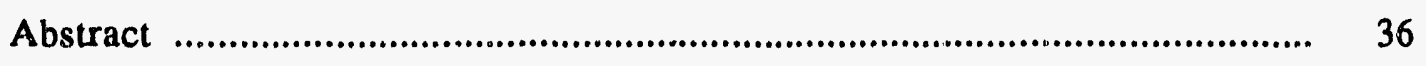

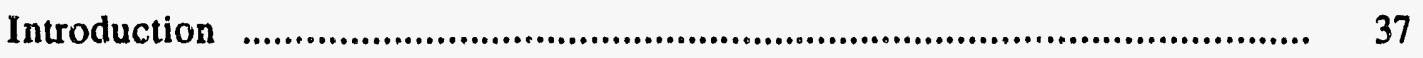

Materials and methods ................................................................... 40

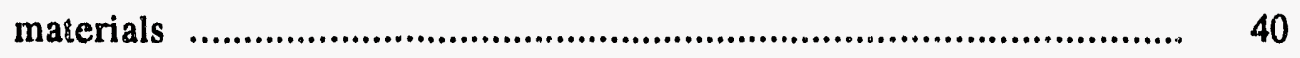

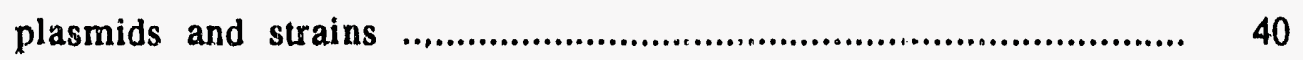

induction of expressed proteins .............................................. 42 


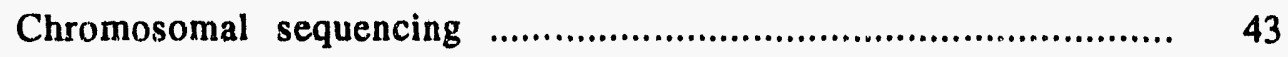

Computational sequence analysis ......................................... 43

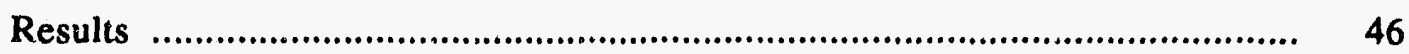

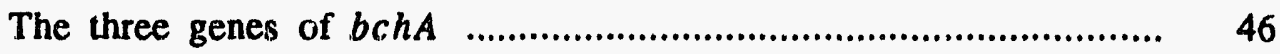

$b c h Y$ and $b c h Z$ are translationally coupled ............................ 51

$b c h Y$ and $b c h Z$ share amino acid identities with other

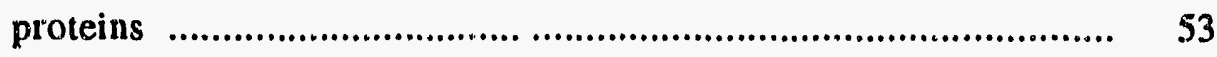

Discussion ..................................................................................... 56

The bchA region contains three genes: $b \operatorname{ch} X, b c h Y$,

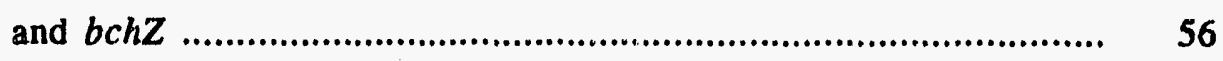

Translational initiation of $b \operatorname{chZ}$ and $b c h Y$............................ 58

Chlorophyll Fe proteins .......................................................... 60

Chapter 3: The bchFNBH bacteriochlorophyll synthesis genes of Rhodobacter capsulatus, and identification of the third subunit of light-independent protochlorophyllide

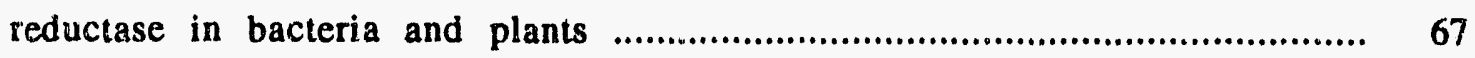

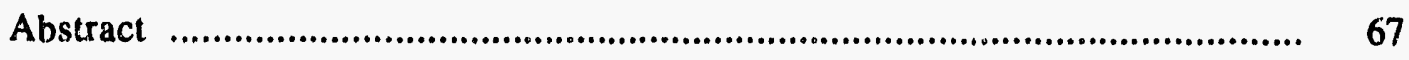

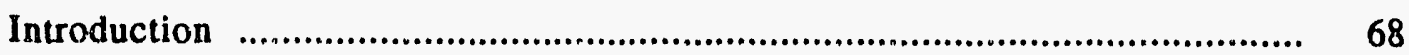

Materials and methods .................................................................... 72

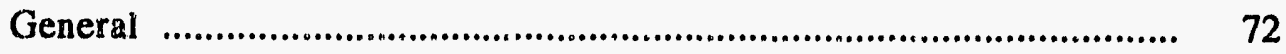

DNA extractions and analysis ............................................. 72

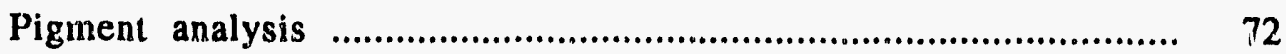

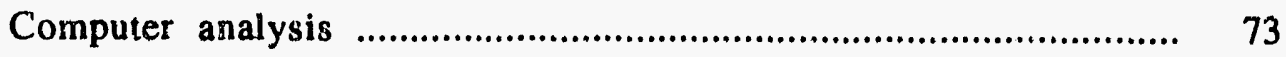

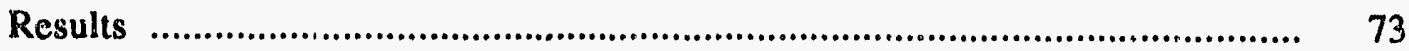

Sequence of the feur open reading frames 
of BamD and identification of potential promoters ............ 73

Mapping the locations of transposon insertion mutations ..... 76

Phenotypes of transposon mutants .......................................... 79

The PChlide reductase subunits have homologs in

plant chloroplasts ........................................................... 85

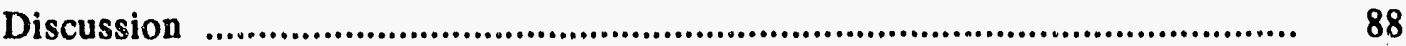

Operon structure and gene assignments ................................ 88

The third subunit of PChlide reductase in plants ..................... 91

Properties of PChlide reductase .................................................. 93

The role of $b c h H$

Chapter 4: The early evolution of photosynthesis and nitrogen fixation.... 98

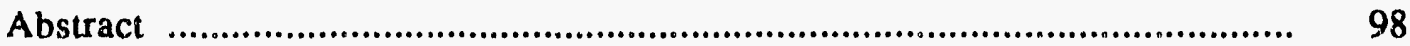

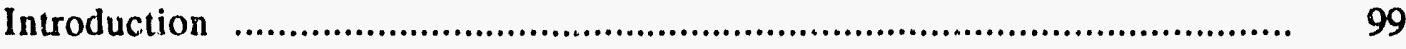

Chlorophyll vs. Bacteriochlorophyll .............................................. 102

Quantitative analysis of the iron protein subunits $\ldots \ldots \ldots \ldots \ldots \ldots \ldots \ldots \ldots \ldots 111$

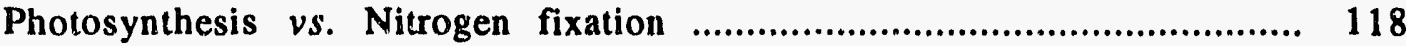

Evolution of reaction centers pigments before (B)Chl .................... 119

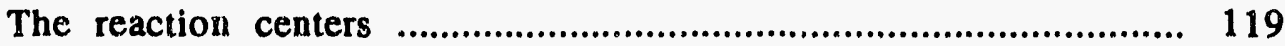

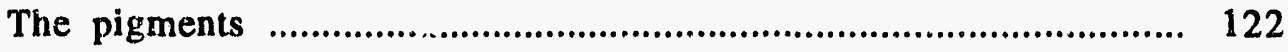

Light independent $v s$. light dependent PChlide reduction ................ 124

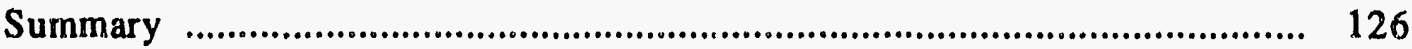

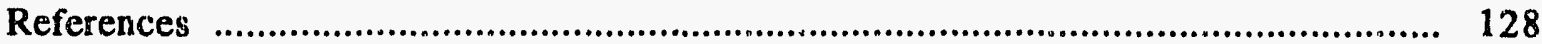




\section{List of tables and figures}

\section{Tables}

3.1 Phenotypes and genotypes of strains used in this study

4.1 Relative similarities of the PChlide reuctase and chlorin reductase subunits

4.2 Strains used in quantitative phylogenetic analysis of nifH and Chl Fe proteins

\section{Eigures}

Chapter 1

1.1 Evolutionary relationships among the 12 Phyla of the Bacterial Domain

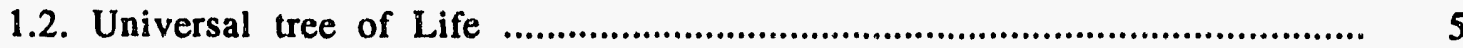

1.3 Charge separation is at the heart of photosynthesis ......................... 9

1.4 Light drives the production of a proton gradient .......................... 13

1.5 Uroporphyrinogen is used in of all tetrapyrroles ........................... 15

1.6 Synthesis of $\mathrm{Chl}$ and $\mathrm{Bchl}$ to protoporphyrin IX .............................. 16

1.7 The four isomers of Uro'gen ......................................................... 18

1.8 Synthesis of $\mathrm{Chl}$ and Bchl from protoporphyrin IX ........................ 20

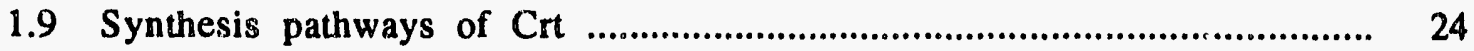

1.10 Pulsed field gel of digesied $R$. capsulatus genomic DNA .................. 29

1.11 Gene map of the photosynthesis gene cluster of $R$. capsulatus ....... 32

\section{Chapter 2}

2.1 Reduction reactions of $\mathrm{Chl}$ and $\mathrm{BChl}$ synthesis .............................. 37

2.2 DNA and deduced amino acid sequences of bchXYZ ............................ 44

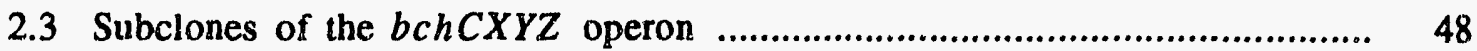


2.4 PAGE-SDS gels of induced proteins ................................................. 49

2.5 Potential secondary structure of intergenic regions ...................... 52

2.6 Alignment of $B c h Z$ sequences from three purple bacteria ................ 54

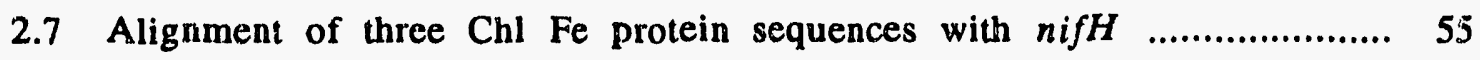

2.8 Proposed mechanism of action of the $\mathrm{Chl} F$

\section{Chapter 3}

3.1 DNA and deduced amino acid sequence of the BamD fragment ........... 74

3.2 Southern hybridization of mapping gel to locate transposons .......... 77

3.3 Spectra of acetone methanol extracts of KZR8G9 and MB1003 ........... 81

3.4 Spectra of acetone methanol extracts of KZR9E11 and KZR9G5 ........... 83

3.5 Alignments of $c h l N-b c h N-b \operatorname{ch} Y$ and $\operatorname{chlB}-b \operatorname{chB}-b \operatorname{chZ} \ldots \ldots \ldots \ldots \ldots \ldots \ldots . . . . . . . . . . \quad 86$

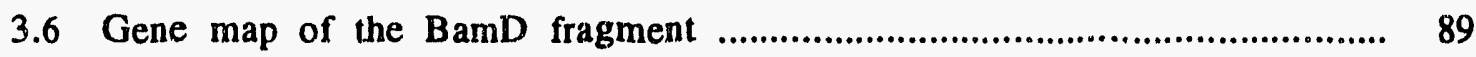

3.7 Hydropathy plot of the N-terminal 1/3 of the $B c h H$ protein .............. 96

\section{Chapter 4}

4.1 Reduction reactions of $\mathrm{Chl}$ and Bchl synthesis .............................. 101

4.2 Possible preservation of gene order among reductase subunits ....... 106

4.2 Phylogenetic distributions of reaction center chromophores

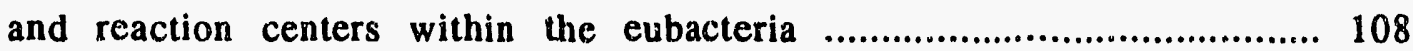

4.3 Structures of the four reaction center chromophores ...................... 109

4.4 Unrooted global phylogenetic tree relating the nitrogenase, PChlide reductase, and chlorin reductase $\mathrm{Fe}$ proteins ...................... 113

4.5 Unrooted tree relating the $\mathrm{Chl} F$

4.6 Two unrooted trees relating the nitrogemase Fe proteins .................. 116

4.7 The root of the $\mathrm{Chl}$ Fe protein tree is on the $s \operatorname{chX}$ line. ..................... 117

4.8 Evolutionary relationships among photosynthetic reaction centers 121 
Chapter 1: Who, What, Where, When, Why, and How

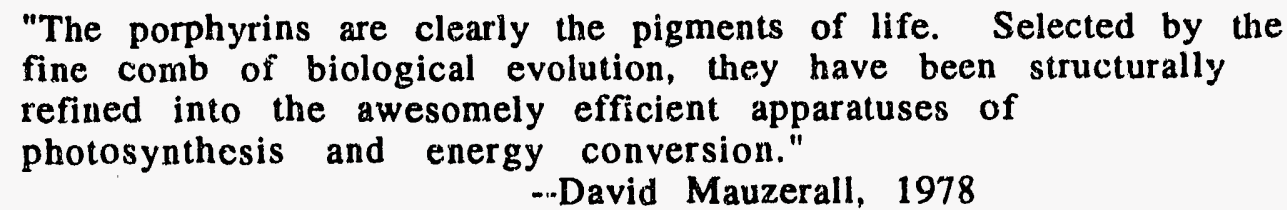

"Then it happened that the leaves began to die. The mighty beasts who appeared to rule the earth were ruled, in truth, by the leaf." --from The Land Before Time

The greatest moment in the history of life on our planet was surely its very inception more than 3.5 billion years ago. Within a very short time, organisms arose whose decedents would drastically alter the face of the earth and make possible life as we know it. These were the photosynthetic bacteria. The prevailing view of the history of the world since then is something like the following:

Near the surface of the global ocean, a single bacterium captured the sun's rays and used the energy to move a proten from one side of its membrane to the other. Soon there were many such bacteria. They spread over all the earth and established complex ecologies of which they formed the foundation. Some of these stripped the electrons from water to feed their photosynthetic engines, giving off oxygen, which was quickly purged from the atmosphere by the iron in the rocks, as a waste product. It took until two billion years ago for the oxygen to saturate the iron and begin to slowly accumulate in the atmosphere. At some point during the next half billion years, one of the water-splitting, photosynthetic bacteria joined with a more complicated, single-celled organism to form the first green alga, from which would later develop the plant world. Another billion years would pass before plants and 
aximals would flourish and diversify in the oceans and begin to encroach upon the land, now shielded from the sur's ultraviolet rays by the orone produced high in the atmotphere from the interplay between the light itself and the increasingly abundant oxygen. The animals fed on the plants that advanced before them and breathed the oxygen that they gave off.

Half a billion : asars later, some of the animals sought to undarstand the workings of one of the photosynthetic bacteria, realizing that to understand photosytuthesis is not only to understand the driving force for nearly all llfe on earth, but to understand some of the very earliest everts in the history of life or earth. It was not one of the water-splitting bacteria, but it was by now nevertheless quite sophisticated in form and function after three and a half billion years of fine-tuning. The work presented in this thesis represents iny efforts over the past few years to advance our understanding of photosynthesis.

The biological context.

Photosynthesis is by all rights strictly a bacterial process. Among modern day organisms, phorosynthetic ability has been found amonis five of the twelve Bacterial kingdoms, or divisions-Heliobacteriaceae, Chlorobiaceae, Chloroflexaceae, Proteobacteria, and Cyamobacteria (fig 1.1) (Woese et al. 1985)--and among the plastid-containing eukaryotes such as plants and algae. It is now widely held that extant life on Earth can be divided into three superkingdoms, or "Domains"--Archae, Bacturia, and Eukaria--all decedent from a common ancestor, or "progenote." The evolutionary relationships among these three domains according to the two major schools of lithought are show in rooted and unrooted forms in fig 1.2 (Gogarten ot al., 1989; lwabe et 


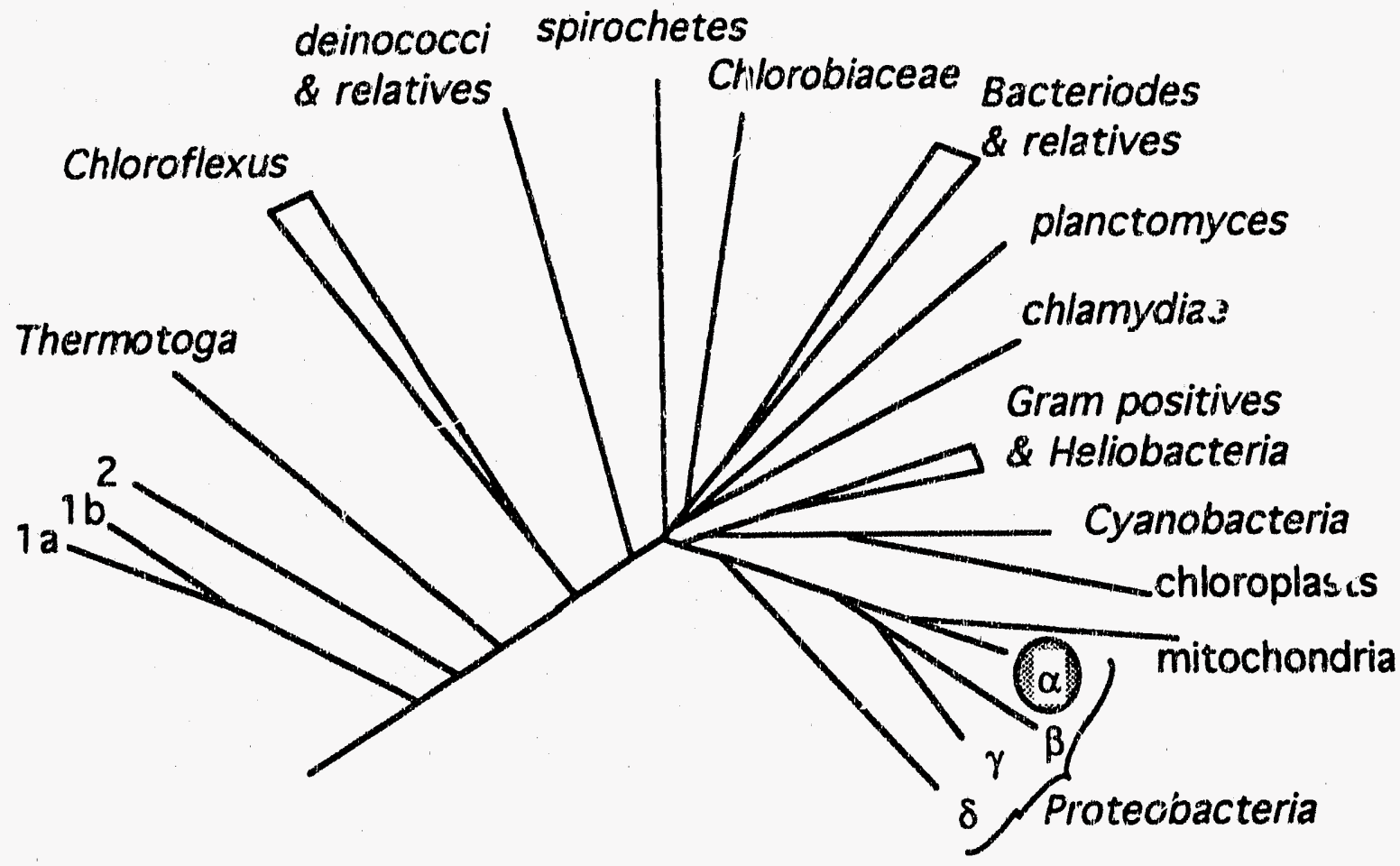

Fig 1.1. Evolutionary reiationsinips among the 12 Phyla of the Bacterial Domain, (based on fig 11 from (Woese 1987)). Branch lengths are proportional to the number of changes in the sequences of each organism's $16 S$ IRNA and therefore reflect the relative evolutionary divergences among the phylogenetic groups shown. Numbers 1 and 2 represent taxa which have recently been discovered but have not yet been described in the literature (N. Pace, personal communication): $\quad \mathrm{a}$ and $1 \mathrm{~b}=$ Electric monk sp.; $2=$ Aquafex. Rhodobacter capsulatus, which forms the core of the studies in this thesis, is a member of the $\alpha$-subdivision of the Proteobacteria, shown highlighted. 
al. 1989, Lake 1991, Rivera and Lake 1992, Woese 1987, Woese, Kandler, and Wheelis 1990) The differences between the two camps and among the various representations shown are given in the legend to the figure. The two traditional kingdoms of Plant and Animal are only minor, peripheral branches (and relatively recent ones at that) within the Eukaria. Eukaryal photosynthesis takes place inside chloroplasts or other similar intracellular plastids. These organelles are decended from free-living cyanobacteria that became the endosymbionts of some ancient eukaryote (Gray and Doolittle 1982), and so even Eukarial photosynthesis is really Bacterial in origin. So far, the only photosynthetic Archae known are the extreme halophilles, which use rhodopsin as their primary photoreceptor molecule instead of a chlorophylllike molecule, and do not produce oxygen. Bacterial photosynthesis is so widely distributed among the various taxa that it may have been present at the time of, or shortly after, the divergence of the superkingdoms (Woese et al. 1985).

The research lab of Prof. John Hearst, along with several other labs, has dedicate $\downarrow$ much of the last decade to studying one member of the $\alpha$-subdivision of the Proteobacteria (also known as the purple bacteria) named Khodobacter capsulatus (a Red Bacterium which forms a polysaccharide capsulus to protect it from its surroundings). $R$. capsulatus has all of the properties of an ideal organism for molecular genetic studies of photosynthesis. When oxygen levels drop, it induces an extensive photosynthetic intracytoplasmic membrane. However, it can also grow aerobically in the presence of oxygen. This dual life-styce reflects the fact that $R$. capsulatus grows along the chemocline (the chemical equivalent of a thermocline) of meromyctic lakes, where it is alternately exposed to oxygen-containing waters from above and 

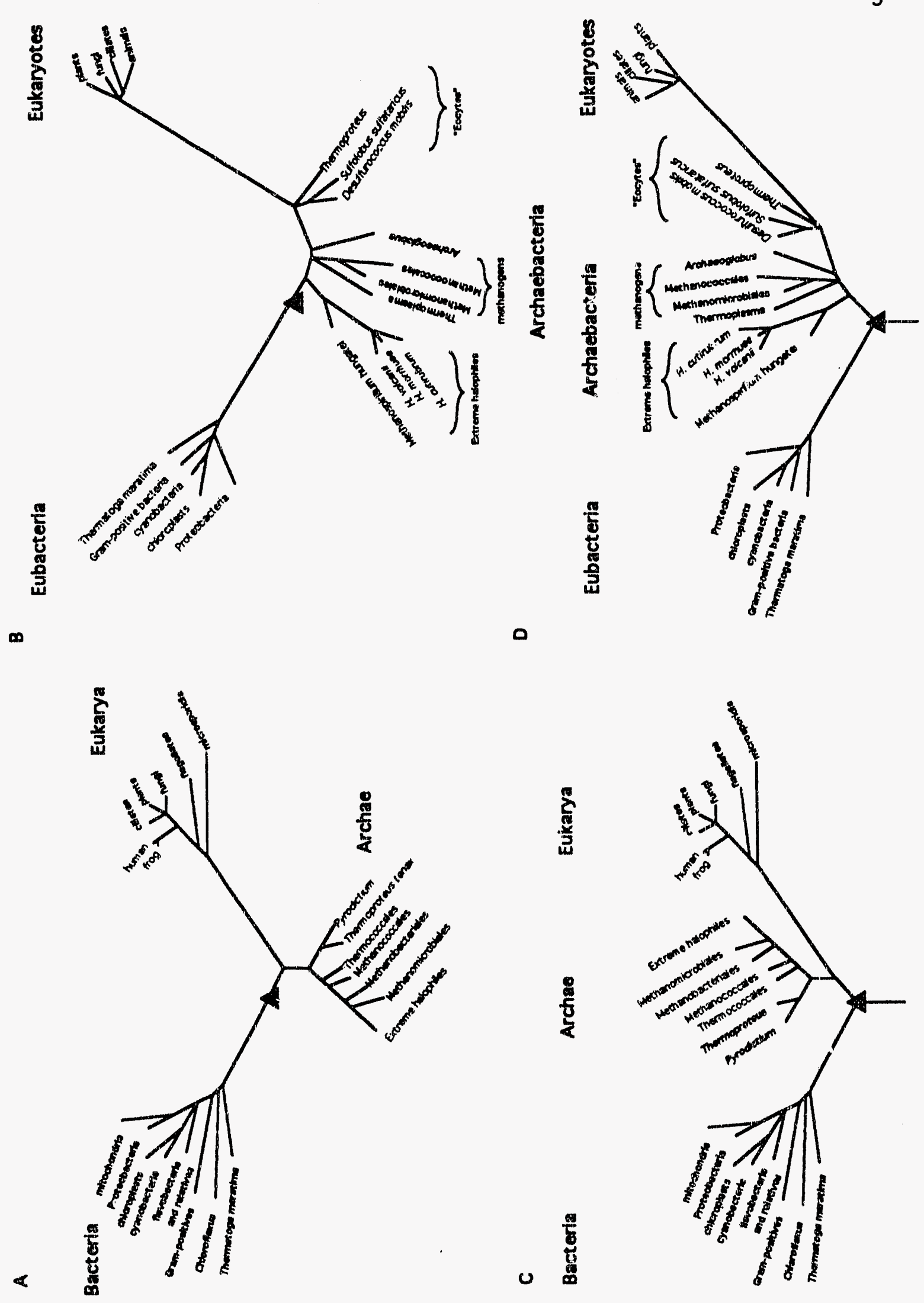
Fig 1.2. Universal tree of Life. All four representations aim to shows the evolutionary relationships among all forms of life on our planet. The length of each line segment is proportional to the number of changes in the sequences of each organism's $16 \mathrm{~S}$ or 18S rRNA. The unrooted forms (shown in a and d) can be thought of as aerial views, where one cannot be sure which part of the branching lies closest to the ground. The point where the tree touches the ground defines the last common ancestor of all the members of the tree. Additional assumptions must be made in order to locate this point, known as the "root" of the tree. The Woese tree (a) (Woese 1987) was rooted by Iwabe et al. (1989) at the shaded triangle by assuming that the $\alpha$ and $\beta$ subunits of ATPases found in all organisms diverged from each other as the result of a gene duplication event prior to the separation of the major lineages. When they compared $\alpha$ and $\beta$ subunit sequences from all three domains, the point on the $\alpha$ tree that was closest to the $\beta$ tree and the point on the $\beta$ tree that was closest to the $\alpha$ tree were both at the shaded circle, suggesting that that was the point at which the two lineages began to evolve independently. Similar results were obtained when the sequences of translation elongation factors EFTu and EF-G were analyzed in this fashion. Lake used a different method of $16 \mathrm{~S}$ rRNA analysis to derive a tighter relationship between the Eukaryotes and the extremely thermophylic, sulfur metabolizing bacteria (Eocytes) (c) (redrawn from (Lake 1991) in an unrooted form. Lake has recently found an 11 amino acid signature sequence in elongation factor EF-1a of both groups which is absent in the other groups (Rivera and Lake 1992), confirming that the Eocytes are our closest prokaryotic relatives. Imposing the Iwabe rooting (shaded triangle) onto the unrooted Lake tree gives the representation shown in part $d$. 
sulfurous, anaerobic waters from below. More importantly for this work, however, the ability of Rhodobacter to dispense with photosynthetic growth allows photosynthetically deficient mutants to be generated and maintained. By means of genetically manipulating the photosynthesis genes of $R$. capsulatus, a large number of mutant strains have been generated which are defective in one or more of the functions required to support photosynthetic growth. A great deal of work has gone into analyzing the consequences of specific gene disruptions in order to fivine the functions of the disrupted genes (for example, see chapter 3).

R. capsulatus and other members of the family Rhodospirillaceae have long been the subject of enzymological, spectroscopic, and genetic studies of photosynthesis. These studies, in addition to being of interest among bacteriologists, have laid the foundation for much of the work now being done in molecular studies of plant photosynthesis. For instance, amino acid sequence comparisons of genes with known functions from $R$. capsulatus with proteins of unknown function has allowed the identification of the plant reaction center proteins (Hearst and Sauer 1984h) (D1 and D2, the so-called "herbicide binding protein" or "quinone-binding protein") while other sequence comparisons have allowed the identification of as many as four chlorophyll synthesis gines (see chapters 2 and 3). Certain aspects of both carotenoid and bacteriochlorophyll biosynthesis pathways were first established in either $R$. capsulatus or in the closely related $R$. sphaeroides. (In fairness it must be pointed out that much of the chlorophyll pathway was worked out by analyzing mutants of the green alga Chlorella.) The crystal structures of the photosynthetic reactions centers from $R$. sphaeroides (Allen et al. 1988a, Komiya et al. 1988b) and Rhodopseudomonas viridis (Deisenhofer 
and Michel 1991) are the only ones that have been determined to atomic resolution, and the $R$. viridis reaction center structure solution earned the 1988 Nobel Prize in chemistry for the principle investigators in that work.

The relative ease of doing genetic work on photosynthesis in $R$. capsulatus is in contrast with similar work in plants, algae, and cyanobacteria. For example, mutations in essential genes for photosynthesis are usually lethal to plants. Recently, two groups of chlorophyll-containing model organisms have become accessible to molecular studies. The single celled alga Chlamydomonas reinhardtii is able to grow nonphotosynthetically; however, whereas $C$. reinhardtii chloroplasts are readily transformed by homologous recombination, it is still impossible to manipulate the genes in its nucleus, where most of the pigment synthesis genes are located. Many species of cyanobacteria are naturally competent for taking up and recombining foreign DNA, but until very recently, there were none that could grow without an intact photosystem. Now such strains have been isolated, but their chemoheterotrophic (i.e., non-photosynthetic) growth is extremely slow, with doubling times ranging from 12 to 36 hours. Chloroflexus aurantiacus grows very weil aerobically in the dark, but there is as yet no genetic system available for manipulating its genes. The other two Bacterial divisions are both obligate anaerobes (i.e., they die or stop growing if exposed to oxygen), they fail to thrive in the dark, and there are currently no genetic systems available for manipulating their genes.

\section{Bacterial Photosynthesis.}

Development of the photosynthetic membrane (reviewed in refs (Drews 1985, Kiley and Kaplan 1988)) requires the de novo synthesis of four 
components: i) bacteriochlorophyll (BChl) pigment molecules to serve as the primary photoreceptor molecules, ii) reaction center proteins, wherein the primary charge separation converting light energy into chemical energy takes place, iii) carotenoid (Crt) pigment molecules to protect the cell from the otherwise lethal photooxidative effects of the excited state BChl, and iv) light harvesting antennae proteins to channel incoming light energy into the reaction center.

The reaction center contains two integral membrane peptides designated $L$ and $M$, which are similar in amino acid sequences and threedimensional structure (Coleman and Youvan 1990, Deisenhofer and Michel 1991). Both $L$ and $M$ contain five transmembrane helices. Indeed, rotation of the reaction center structure by $180^{\circ}$ about an axis perpendicular to the plane of the membrane allows for the superposition of many of the residues in the $L$ subunit onto the corresponding residues in the $M$ subunit, and vice versa. Some species, including $R$. capsulatus, contain an additional subunit designated $\mathrm{H}$, which is largely extrinsic to the membrane on the cytoplasmic side but contains one transmembrane helix. The most conserved sequences among $H$ protein subunits are involved in protein-protein contacts with the $L$ and $M$ subunits (Berard and Gingras 1991). Cyanobacteria, algae, and plants also utilize heterodimeric reaction centers in both photosystems I (PSI) and II (PSI). PSI-like RCs are found in the Chlorobioaceae and Heliobacteriaceae, while the RCs of Chloroflexus and the purple bacteria (i.e., the Proteobacteria) are PSII-like (Olson and Pierson 1987, Pierson and Thornber 1983a). The PSII reaction center proteins $D 1$ and $D 2$ are very similar in sequence to the $L$ and $M$ peptides. Although all four peptides probably share a common ancestor, the exact evolutionary history is probably more complicated than has been 


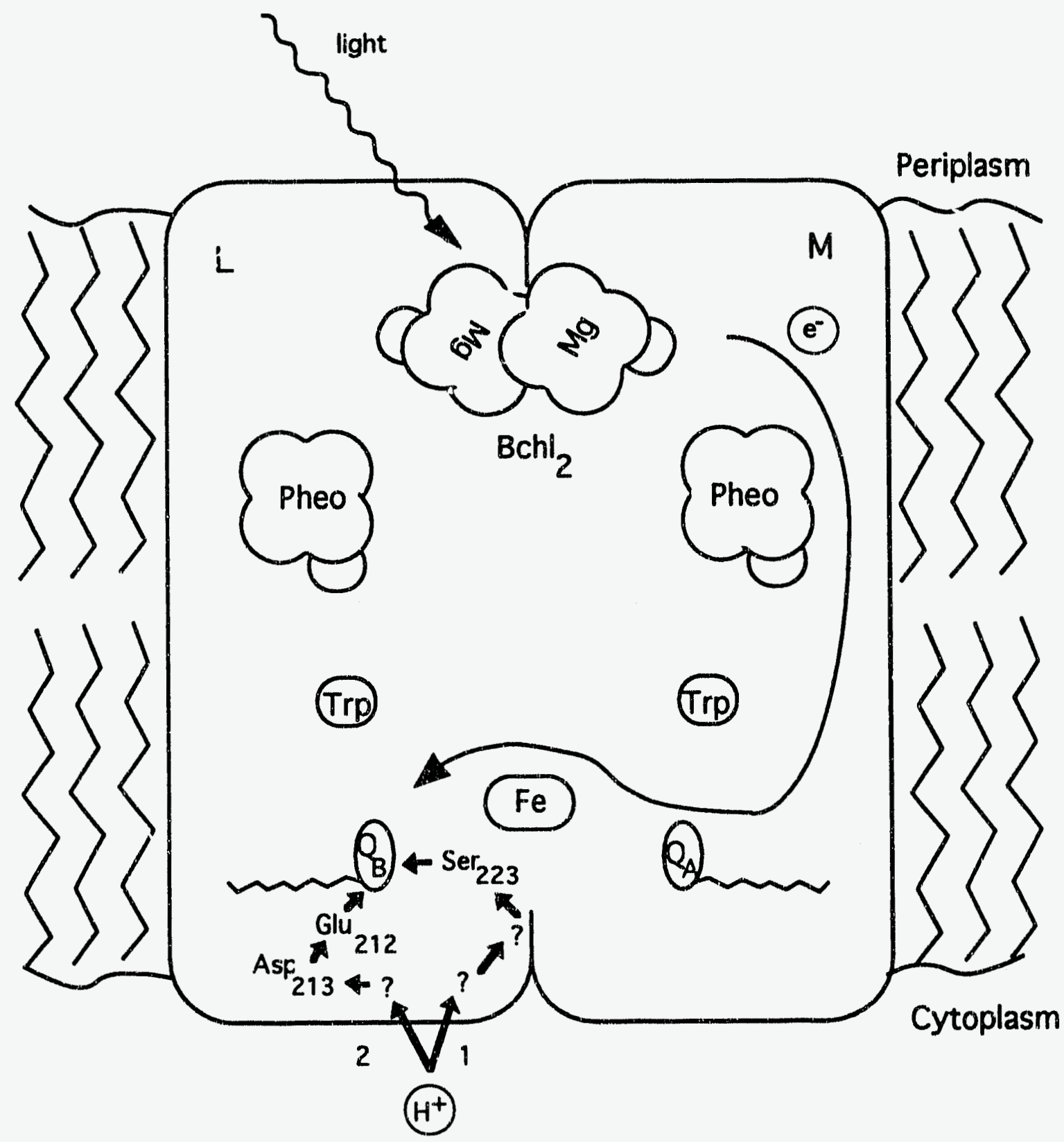

Fig 1.3 Charge separation is the heart of photosynthesis. See text for details. 
previously suspected (see chapter 4). In addition to their similarities, the PSII reaction center differs from the purple bacterial reaction center in that i) it binds chlorophyll in place of bacteriochlorophyll, ii) it contains several small insertions of "extra" peptide sequences in each protein, and iii) it is the site where water is reduced into $\mathrm{O}_{2}$ and protons, a process which takes place only in cyanobacteria and their relatives (such as chloroplasts). Investigation into the structural basis of water splitting is yielding answers, albeit very slowly; thus far the details of the mechanism remain a mystery.

The cascade of chemical reactions of photosynthesis begins with the absorbance of a photon by the "special pair" of bacteriochlorophyll (Bchl 2 ) molecules in the reaction center (fig 1.3). The excitation energy of the photon most often reaches the reaction center by direct transier from the antenna. A single electron is then transfered from the excited special pair to a bound bacteriopheophytin (a $\mathrm{BChl}$ without its central $\mathrm{Mg}$ atom), then through a Trp residue in the $M$ protein to a quinone molecule bound into the " $A$ " site $(Q) A$. and finally to another quinone bound in the " $B$ " site $(Q) B$ on the other peptide. The net result is that an electron is transfered across the membrane from the special pair to $(\mathrm{Q})_{\mathrm{B}}$, forming a semiquinone radical $\left(\mathrm{Q}^{-}\right)_{\mathrm{B}}$ and leaving behind 2 positively charged special pair radical $\left(\mathrm{Bchl}_{2}\right)^{++}$. In spite of the overall symmetry of the $L$ and $M$ subunits, electron transfer from the special pair to (Q)B occurs strictly along the $D$ helix of the $M$ subunit, never along the nearly identical $D$ helix of the $L$ subunit. The basis of asymmetric electron flow is still hotiy debated, but subtle differences in configuration of the pigments and their immediate protein environments may hold the key. When an electron is transfered from soluble cytochrome $c_{2}$ into the special pair, the positive charge of the special pair radical is neutralized and the unpaired electron is 
paired. The negative charge on the quinone radical is neutralized by transfer of a proton from a nearby Ser residue $(\# 223)$ on the $M$ subunit between the (Q)B site and the cytoplasm (fig 1.3) (Paddock et al. 1990). The reaction center is then ready to transfer another electron to $Q_{A}$ and on to $Q_{B} H$. This time the resulting $\mathrm{QBH}^{-}$is neutralized by transfer of a proton from the nearby Glu212, which in turn picks up a proton from Asp213 (Paddock et al. 1990, Paddock et al. 1989), which is still closer to the cytoplasm. Ultimately, the source of all the protons for the "proton relay" is the cytoplasm itself.

Once another proton has been taken up from the proton relay system, hydrcxyquinone $\left(\mathrm{QH}_{2}\right)$ slips out of the reaction center and into the lipid phase of the membrane, where it diffuses to an integral membrane cytochrome complex known as cytochrome bc 1 . Cytochrome $b_{1}$ transfers two electrons from $\mathrm{QH}_{2}$ to two cytochrome c2 molecules and releases two protons to the periplasmic side of the membrane. The regenerated quinone (Q) diffuses back through the membrane, where it is readily taken up by a reaction center with an empty $(Q) B$ site, while cytochrome $c_{2}$ goes off in search of $B C h l$ special pairs in need of electrons (fig 1.4).

Thus electron flow in purple bacterial photosynthesis is cyclic (special pair $\rightarrow>$ quinone $\rightarrow>$ cyt bc $1->$ cyt $c_{2} \rightarrow>$ special pair), while proton flow is unidirectional (cytoplasm -.> "proton relay" -.> quinone.$->$ periplasm), and so protons build up on the periplasmic side of the membrane (fig. 1.4). Invagination of the membrane into little pockets both increases the total surface area of the cell that can be dedicated to photosynthesis and helps to keep the local periplasmic concentration of protons high. The proton gradient generated by this mechanism is what drives the cell's chemical 


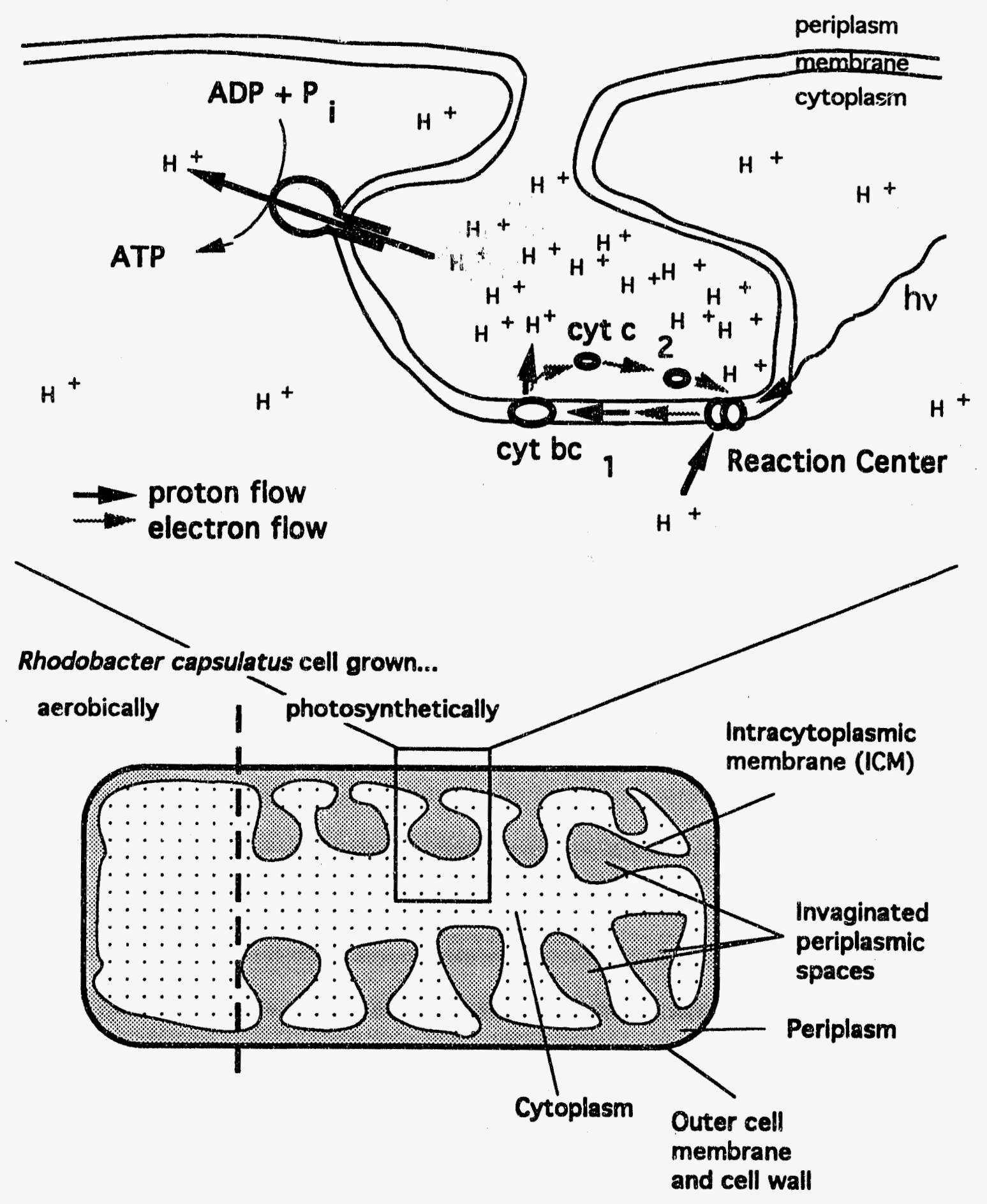

fig 1.4 Light drives the production of a proton gradient; the release of the energy buildup produced by that gradient as protons return to the cytoplasm through special channels drives the synthesis of ATP, which powers most of the cell's metabolic processes; hence the term "Photo-Synthesis." 
reactions. Special proton channels regenerate ATP from ADP and free phosphate driven by the spontaneous flow of protons from the periplasmic side of the intracytoplasmic membrane down the concentration gradient back into the cytoplasm.

\section{Photosynthetic pigments.}

None of the above reactions could take place without pigments. Both bacteriochlorophyll ( $\mathrm{BChl}$ ) (or chlorophyll, $\mathrm{Chl}$, in green photosynthesizers) and carotenoids (Crt) are synthesized by all modern photosynthetic organisms (excluding the unrelated halophillic bacteria mentioned before). More than three-fourths of the genes specifically required for photosynthesis in $R$. capsulatus are involved in production of one of these two pigments. Below I outline the important features of their biosynthetic pathways. Chapters two through six then detail my efforts to make sense of the genes required for BChl synthesis.

Bacteriochlorophyll. The biosynthecic pathways of $\mathrm{BChl}$ and $\mathrm{Chl}$ can be divided into three segments: precursors to uroporphyrinogen III, uroporphyrinogen III to protoporphyrin IX, and protoporphyin IX to either BChl or Chl. Various parts of the (B)Chl synthesis pathway are shared with all of the tetrapyrrole compounds (fig 1.5), as heme (the prosthetic group of hemoglobin and cytochromes), biliverdin(a bile pigment and breakdown product of heme), the phycobilins (used in cyanobacterial photosynthetic antennae complexes), Vitamin B12 (a cofactor for an enzyme that converts methylmalonate-CoA to succinyl-CoA), factor $F_{430}$ (a cofactor used in methane production in methanogenic archaebacteria), and siroheme (the prosthetic group for nitrite and sulfite reductases) (Warren and Scott 1990). It is likely 


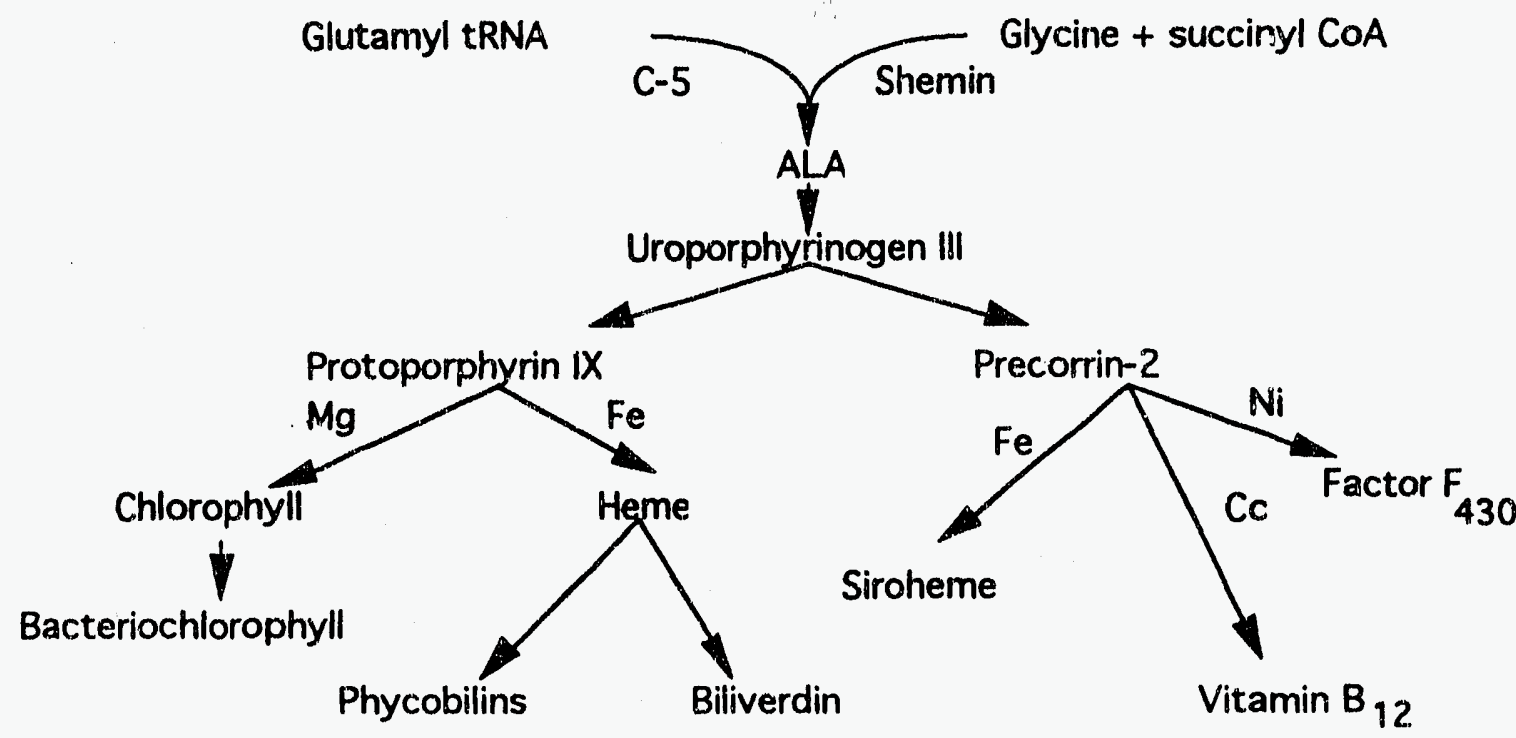

fig 1.5 Uroporphyrinogen is used in the synthesis of all tetrapyroles.

that most of these compounds are derived functions that arose as branchings of whichever pathway came first in the remote evolutionary past.

The early part of the biosynthetic pathway of $\mathrm{BChl} / \mathrm{Chl}$ is shown in f:gure 1.6 and is reviewed in references (Beale and Weinstein 1991, Leeper 1991). The most intensively studied step in the scheme shown is the first, namely, the formation of $\delta$-amino-levulinic acid (ALA). All organisms make ALA, but there are two pathways by which they can do so. The most prevalent is the C-5 pathway, in which glutamyl-tRNA is reduced to glutamate-1semialdehyde(shown in three resonance forms) plus tRNA, followed by rearrangement to ALA catalyzed by pyridoxyl-P-aminotransferase. The 
Synthesis of ALA, Uro'gen III, and Protoporphyrin IX
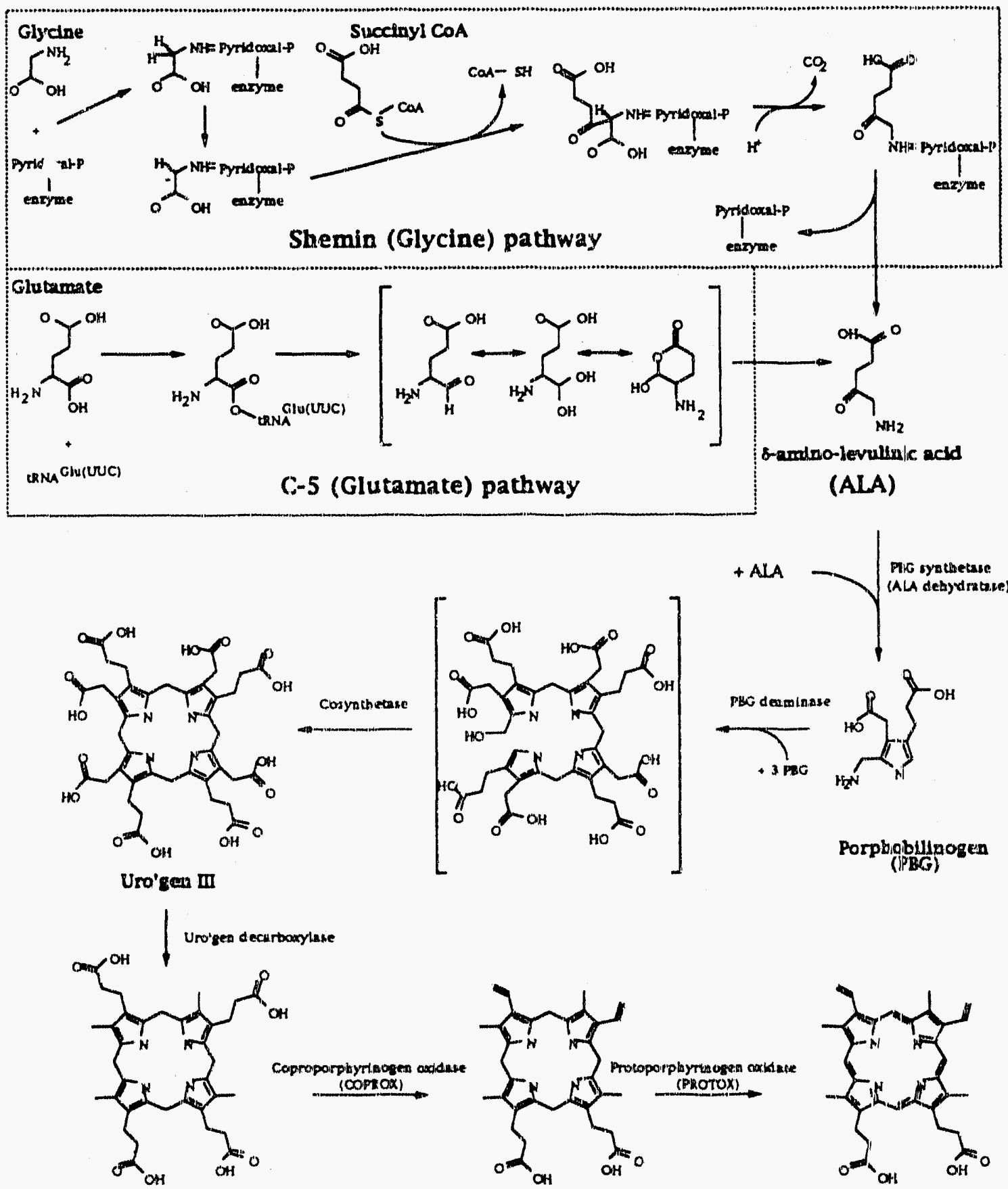

Copro'gen

Proto'gen

Protopiorphyrin IX 
glutamate used in the reaction is activated by linkage to IRNAGI". The use of IRNA as a leaving group has been postulated as being a remnant of the enzymology of the RNA world, a time before protein-based life when RNA molecules are thought to have been the key biochemical pliyers in both storing genetic information and carrying out the cellular functions (Gilbert 1986), since in principle, any of many other leaving groups could take the place of tRNAGlu (Benner, Ellington, and Tauer 1989). The second pathway, which is largely limited to mitochondria and to the $\alpha$-subdivision of the Proteobacteria (Avissar, Ormerod, and Bealc 1989, Beale and Weinstein 1991), is the so-called "Shemin" pathway. (Note: Mitochondria are decended from freeliving bacteria that are derived from the $\alpha$-subdivision of the Proteobacteria much the same way that chloroplasts are derived from free-living cyanobacteria. Thus the congruence of metabolic pathways utilized is not surprising.) In this reaction, condensation of glycine and succinyl-CoA yields a 6-carbon intermediate which, upon decarboxylation of the $\alpha$-carbon derived from glycine, yields ALA. Both the C.-5 and the Shemin pathways to ALA formation are inhibited by ALA and by heme, and so feedback regulation is proposed to be a major determizant of how much ALA enters the tetrapyrrole synthesis pathway (Beale and Weinstein 1991).

Two molecules of ALA are condensed by ALA dehydratase (more properly refered to as PBG synthetase) to form porphobilinogen, or PBG. PBG is a flat, aromatic compound with three side chains: aminomethyl, acetic acid, and propionic acid. It is the monomeric unit of which there are 4 in a "tetrapyrrole" compound such as heme or bacteriochlorophyll. Condensation of four PBG units into a macrocyclic ring yields a "uroporphyrinogen." 


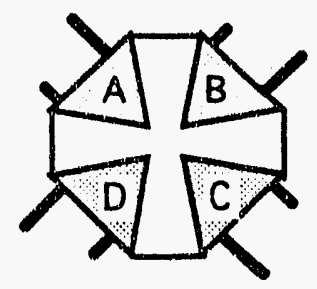

Uro'gen 1

.125

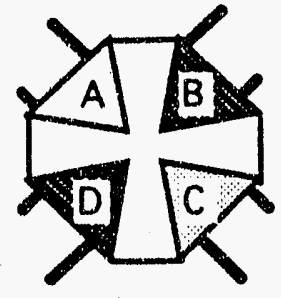

Urc'gen II

.125

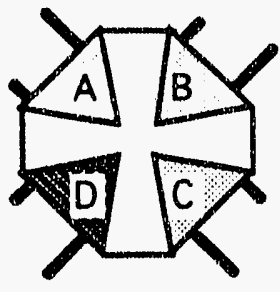

Uro'gen III

.500

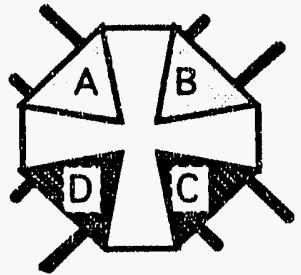

Uro'gen IV

.250

fig 1.7. The four isomers of Uro'gen.

Because of PBG's asymmetry, there are four distinct ways in which four PBG units can be cyclized into uroporphyrinogen, and these are designated uro'gen I-IV. (This and the next two compounds in the pathway all contain the root "porphyrinogen" in their names, which is commonly shortened to “"gen.") Fig 1.7 shows schematically the arrangement of PBG units in the four uro'gens, where the short stern represents the acetic acid group and the long stem the propionic acid group. The numbers under each figure refer to the fraction of each that would be formed if their assembly were random. Uro'gen III is the physiologically relevant isomer, and it is the most readily formed of the four isomers under nonenzymatic conditions (Mauzerall 1960). A twosubunit enzyme is required for the conversion of PBG into uro'gen III. In the presence of only PBG deaminase (without the "CoSynthase") uro'gen I is the only isomer formed, as can be seen by closing the macrocyclic ring in the intermediate shown in brackets in fig 1.6. The Cosynthetase flips ring $D$ before allowing closure to take place. (See refs (Leeper 1991, Warren and Scott 1990) for the proposed mechanisms of this ring flip.) Uro'gen III is the first compound in the pathway to possess the macrocyclic four ring structure of the porphyrins. It is also the branch point for the synthesis of all tetrapyrrole 
compounds and is thus the last compound in this pathway synthesized by all living organisms (Leeper 1991, Warren and Scott 1990).

All four acetic acid side chains of Uro'gen III are decarboxylated to methyl groups by uro'gen decarboxylase to produce copro'gen (i.e., coproporphyrinogen). Subsequent decarboxylation of copro'gen rings $A$ and B occurs oxidatively, leaving vinyl, rather than ethyl groups at these positions in Proto'gen. (The full name of the enzyme that catalyzes this reaction is ceproporphyrinogen exidase. This has been shortened successively to cepro'gen exidase, and finally to COPROX.) Finally, Proto'gen is oxidized by PROTOX to form the fully aromatic protoporphyrin IX. The oxidation state of the macrocycle goes in this step from the "porphyrinogen" level to the "porphyrin" level, while the total charge has gone from -8 to -2 . Both of these changes are in the direction of making the molecule more useful for photosynthesis, since the negative charge is difficult to bury within the hydrophobic environment of the membrane, and aromaticity shifts the absorbance maximum closer to the visible and allows for stabilization of the special pair by charge delocalization once it donates an electron into the charge transfer system.

The important chemical conversions of the chromophore that take place over the course of the $\mathrm{Mg}$ branch are shown in fig 1.8 , along with the genes responsible for these conversions in $R$. capsulatus. The gene assignments shown are compiled from previous work (Biel and Marrs 1983, Taylor et al. 1983, Yang and Bauer 1990, Yen and Marrs 1976, Zsebo and E. 1984a) and from chapters 2 and 3 of this thesis. In these final steps, the macrocycle in converted from a porphyrin into a pheoporphyrin, from there 
Chlorophyll and Bacteriochlorophyll biosynthetic pathways

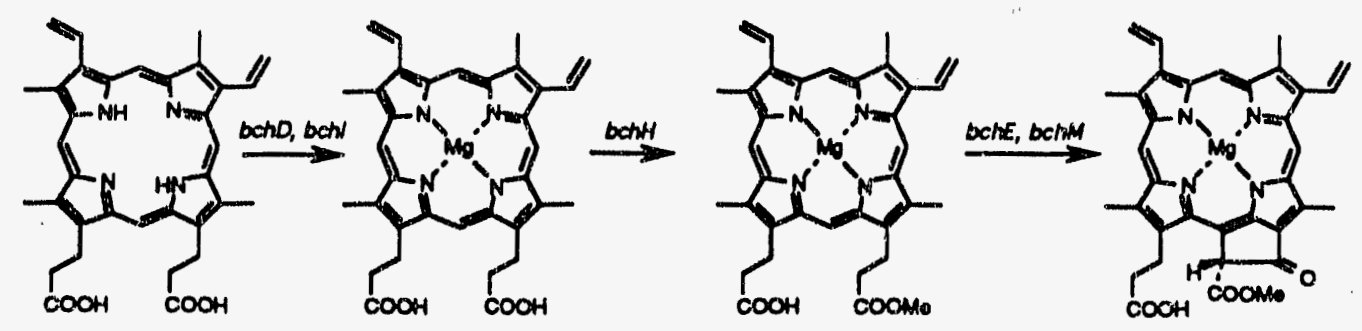

Protoporphyrin QX Mg-Motromethyl Protoporphyrin ix Mg-Alonomethyl Protoporphyrin LX Mz-Divinyl Pratochlorophyllide
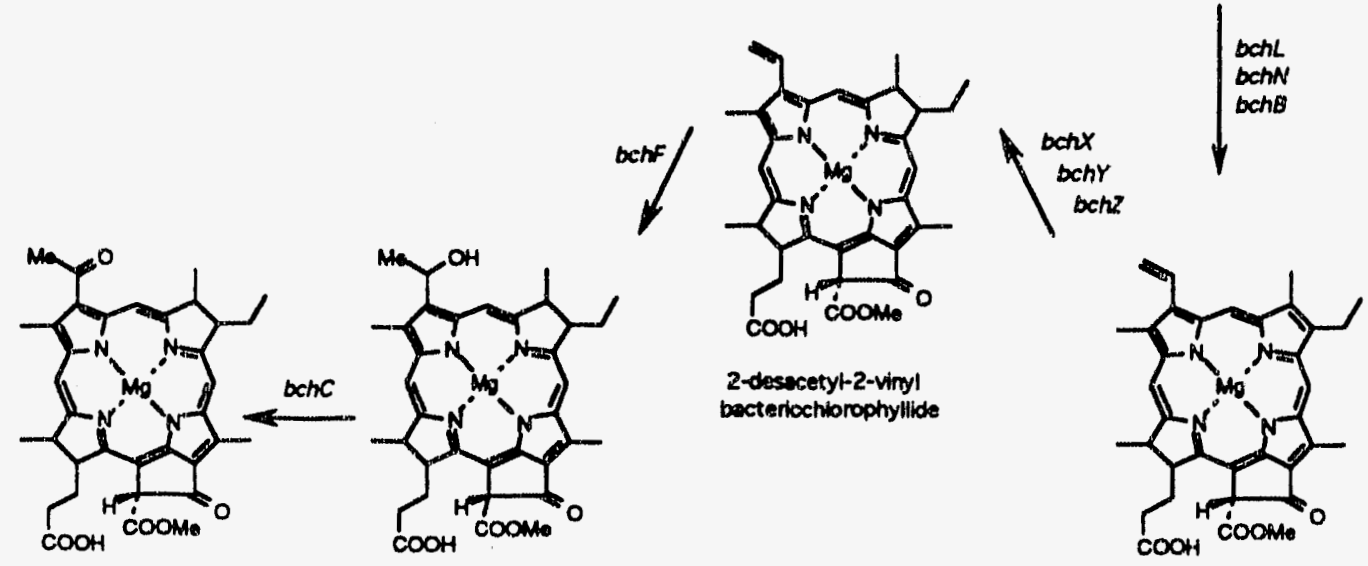

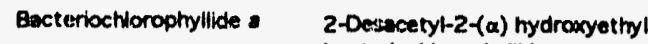
bocteriochlorophyllide a
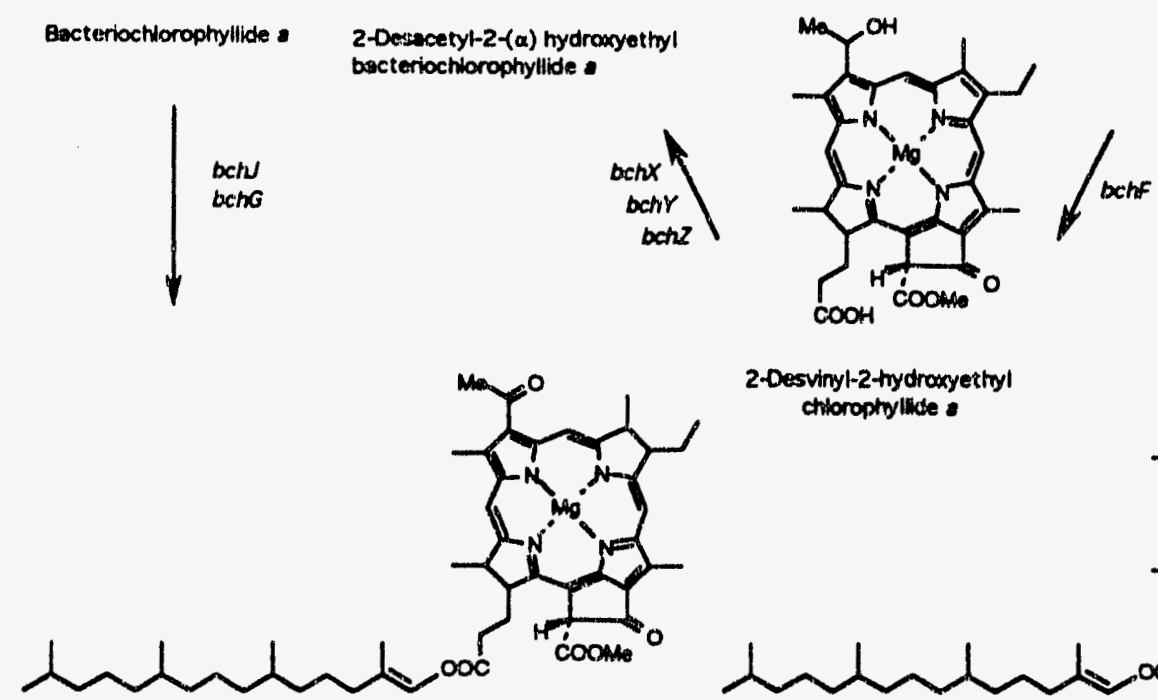

Chiorophylitide a

$$
\begin{aligned}
& \text { 2-Desvinyl-2-tyddraxyethyl } \\
& \text { chiorophyllide }
\end{aligned}
$$

Bacteriochlorophyll a

Chiloroplityll a

fig 1.8 
into a chlorin, and finally into a bacteriochlorin. Of equal importance is the neutralization of the two remaining negative charges to facilitate burial within the greasy, hydrophobic environment of the membrane. Decorations of the side chains extending from the macrocycle give each of the various Chls and Bchls their special identities.

Protoporphyrin IX is the only naturally occuring porphyrin without a metal. $R$. capsulatus and other photosynthesizers produce protoporphyrin IX as a precursor not only for $\mathrm{BChl}$, but also for the hemes that it uses in its cytochromes. Specific incorporation, or "chelation," of magnesium is the last major branch point in $\mathrm{Chl} / \mathrm{BChl}$ synthesis, since insertion of iron into Protoporphyrin IX forms heme. The propionic acid of ring $\mathrm{C}$ is methylated following $\mathrm{Mg}$ chelation in chloroplasts, while chelation and methylation appear to be obligately coupled in $R$. capsulatus (see ref (Gorchein 1972) and chapter 3). Next, the modified propionate of ring $C$ rotates back and joins with the macrocycle in an oxidative step to form a fifth ring, designated ring $E$. The proper name of this compound, "Mg-monomethyl pheoporphyrin a5," is often supplanted with the slightly less cumbersome "protochlorophyllide," or "PChlide," which are the terms used throughout this thesis. Characterization of the genes responsible for the reduction of PChlide to chlorophyllide (Chlide) is the major emphasis of chapter 3 , while those involved in the subsequent reduction of Chlidea to a BChlide are the focus of Chapter 2. Two additional modifications are required to form Bchlidea. Addition of a long hydrophobic tail to Bchlide results in bacteriochlorophyll, while the same addition onto Chlide yields chlorophyll. 
The tetrapyrrole synthesis pathway is of interest to a large number of people not otherwise concerned with photosynthesis, including medical practitioners and farmers. Porphyrias are a class of human diseases in which heme synthesis is faulty. In fact, PBG was first isolated from a porphyria patient before it was later shown that radiolabeled PBG could be incorporated into heme. Other forms of porphyria result in the accumulation of each of the various porphyrinogens (Leeper 1991). In the presence of oxygen and light, these compounds can lead to the production of excited-state singlet oxygen, which in turn causes free radical damage to the cell, including the possible formation of tumors. Porphyria patients are therefore exquisitely sensitive to ultraviolet light, and must avoid exposure to sunlight. The phototoxicity of tetrapyrroles has even led to their use as photosensitizers in medicine. Photosensitizer research is one of the leading current medical application of studies on tetrapyrrole compounds. A cream of chlorophyll precursors (usually a pheophorbide such as PChlide) can be applied topically to a skin tumor, where it is preferentially absorbed by the sumor cells. Exposure of the region to strong light kills the cells that have absorbed the chlorophyll, leaving a large scab reminiscent of a chemical burn. Under the most favorable of conditions, there is no tumor tissue left when the scab clears (Lee et al. 1990, Park et al. 1989). There is a class of herbicides, the diphenylethers, which inhibit PROTOX. Oddly enough, the compound accumulated by plants treated with diphenylether herbicides is not the substrate proto'gen, but rather the product, protoporphyrin IX, which is produced by slow, spontaneous oxidation of proto'gen. Due to compartmentalization of the enzymes within the cell, the accumulated protoporphyrin IX is not accessible to wat otherwise functional enzymes which would most gladly convert it into heme or $\mathrm{Chl}$ In sufficiently dim light, the plants continue to live, but in 
bright sunlight, they are quickly killed by the phototoxic effects of singlet oxygen production (Matringe et al. 1989).

Carotenoids. Free radicals are generated not only in the unusual cases noted above, but also during normal photosynthesis. Although carotenoid (Crt) pigments are also involved in transfer of excitation energy into the reaction center special pair (Trautman et al. 1990), their primary function is to quench these free radicals before they can do any harm. They protect cells from photooxidative damage both directly (by absorbing the ultraviolet rays of the sun) and indirectly (by scavanging free radicals). They are synthesized by all photosynthetic organisms, including the halophilic Archae that use rhodopsin in their light-driven proton pumps, as well as many nonphotosynthetic organisms, including fungi and many bacteria. Ingested Crt are used as pigmentation in lobsters, flamingos, and "nches (along with many other animals), as well as being the major source of Vitamin $A$ (from $\beta$ carotene), from which the visual pigments are synthesized.

Carotenoids contain a forty-carbon skeleton synthesized from fivecarbon isoterpenoid units (fig 1.9). Isopentyl pyrophosphate (IPP) and its isomer, dimethyl allyl pyrophosphate (DMAPP) condense to form geranyl pyrophosphate (GPP). Addition of another IPP unit results in farnesyl pyrophosphate (FPP). FPP finds its way into many important cell functions. The covalent addition of a farnesyl group onto proteir 3 has only recently been recognized as an important regulatory step in directing proteins to the membrane in eukaryotes. FPP is a precursor to the synthesis of hopanoid compounds (a lipid found in some bacteria) and the related steroid hormones, 
Crt biosynthetic pathway

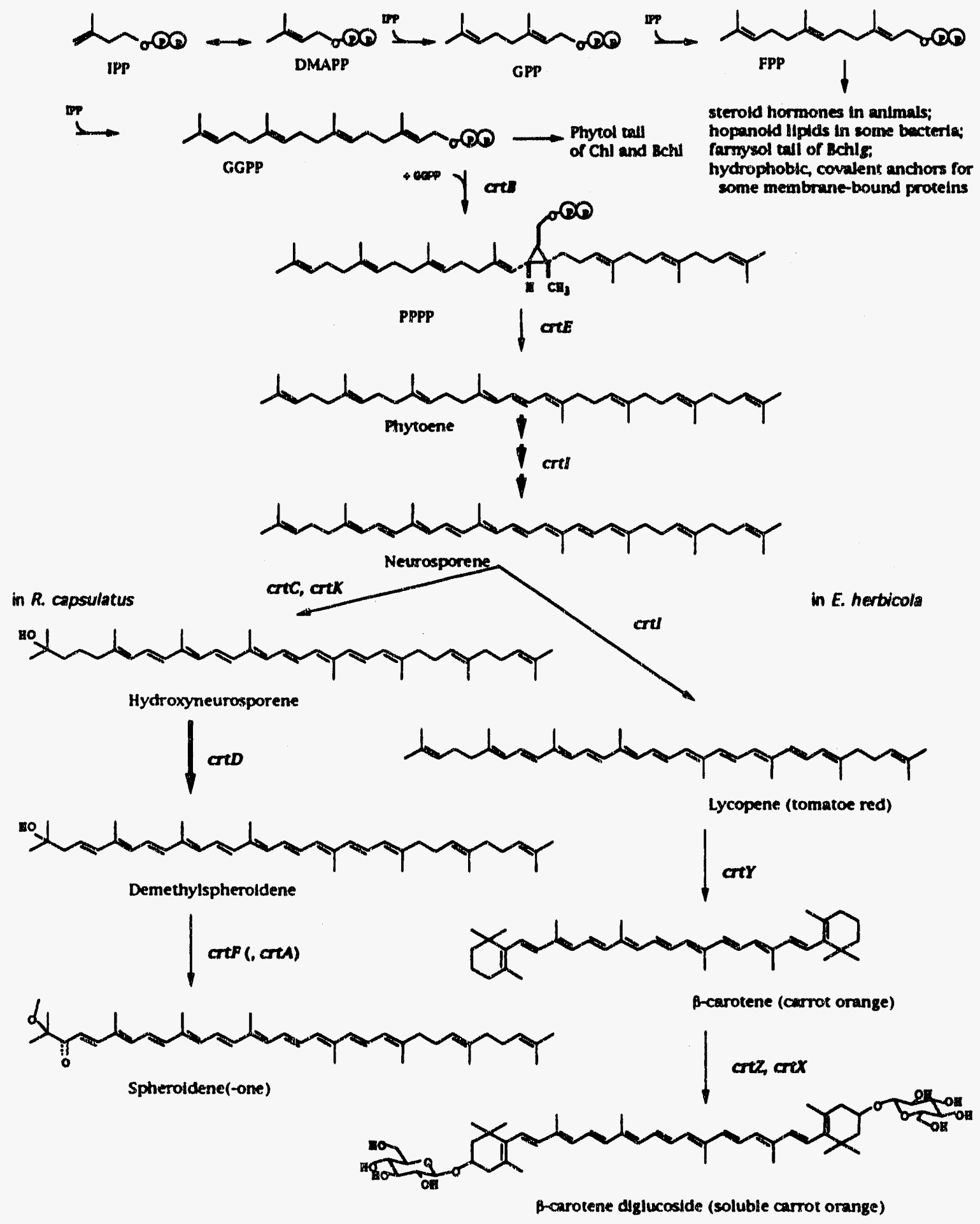


including cholesterol. One strategy that has been proposed for reducing high cholesterol levels has been to inhibit the enzyme that synthesizes FPP.

Addition of another IPP unit to FPP forms geranylgeranyl pyrophosphate (GGPP). Most photosynthetic bacteria add GGPP onto the propionic acid group of BChlide in the last step in the synthesis of BChl, with the exception of the Heliobacteria, which use FPP in synthesizing Bchlg. The long hydrophobic tail anchors the $\mathrm{BChl}$ in the lipid phase of the membrane. Two GGPP molecules then condense into the first $\mathrm{C}_{40}$ compound in the pathway, prephytoene pyrophosphate (PPPP). Loss of the phosphate and rearrangement yields the first true carotenoid, phytoene. Sequential dehydrogenation of phytoene then follows in all carotenogenic organisms, but the details of what $\mathrm{Cr}$ is produced vary tremendously, depending on which organism-specific modifications take place.

The pathways shown in fig 1.9 outline Crt synthesis in $R$. capsulatus, in the non-photosynthetic bacterium Erwinia herbicola, and in many plants. Three dehydrogenations convert phytoene to neurosporene. In Rhodobacter species, neurosporene is hydroxylated at one end before addition of a methyl group onto the oxygen and one additional dehydration to form spheroidene (a

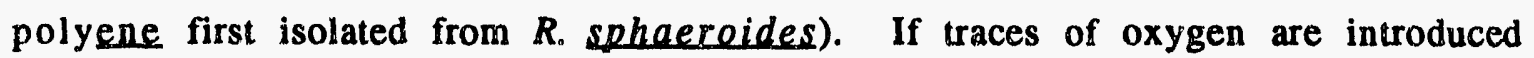
into a photosynthetically growing $R$. capsulatus culture, molecular oxygen is added to spheroidene to form the keto-derivative, spheroidenone (the dotted double bond on the far left end of spheroidine in figure 1.9). In plants and many bacteria, neurosporene is dehydrogenated once more to lycopene, which is then cyclized at each end to form $\beta$-carotene. The difference in color between a tomato (which gets its color from lycopene) and a carrot ( $\beta$ - 
carotene) is only that in the latter, the last six carbons on each end are bent around into a ring. Finally, the non-photosynthetic plant pathogen, $E$. herbicola, produces a more readily soluble form of $\beta$-carotene which has a glucose molecule added onto each ring.

There are nine genes for $\mathrm{Crt}$ synthesis in $R$. capsulatus, eight of which are clustered together into four transcription units (Armstrong et al. 1989a). Our lab has also been involved in the analysis of a crt gene cluster from $E$. herbicola, which presumably uses its $\mathrm{Cr}$ as a general-purpose sunscreen while it munches on the surfaces of the leaves and fruit where it lives. My only work with Crt synthesis has been to initiate a project that ultimately established the functions of all of the Erwinia crt genes, as well as a brief foray into attempting to isolate crt genes from cyanobacteria using PCR. The Erwinia work was primarily carried out by Dr. Bhupinder Hundle. Although it will be presented elsewhere, it is not included in this thesis.

\section{The chromosomal context.}

All of the genes that are specifically required for the production of the ICM in Rhodobacter capsulatus, including those for the reaction center and LHI structural polypeptides, as well as those for the Crt and BChl synthesis enzymes, are located within a contiguous 46-kilobase pair (kb) stretch of DNA. After the initial gene mapping efforts nearly ten years ago (Biel and Marrs 1983, Taylor et al. 1983, Yen and Marrs 1976, Zsebo and E. 1984a), the organization of the photosynthetic (PS) gene cluster was defined in terms of the locations of mutations which gave rise to certain phenotypes. Similar efforts have more recently established that essentially the same organisation is present in the closely related Rhodobacter sphaeroides (Coomber et al. 1990) 
and in the more distantly related Rhodospirillum centenum (Yildiz, Gest, and Bauer, in press). Conservation of this clustering may be related to the relatively facile lateral transfer of all of the photosynthetic functions (and thereby of photosynthetic ability under favorable conditions) from one bacterium to another. Such a transfer event would give a selective advantage to the recipient organism. A clustered set of genes would be more readily transfered in this fashion than genes dispersed in various regions around the chromosome. The net result could be a selective advantage for clustered, vs. non-clustered genes. Note that the entity being selected is not so much the organism receiving the photosynthesis genes, but rather the clustered genes themselves, thereby favoring the clustered arrangement of genes. This type of thinking has been around for awhile, but there is so far no direct evidence in support of the theory and there is some evidence against it. For instance, when all of the $46 \mathrm{~kb}$ gene cluster is introduced into $E$. coli, $E$. coli does not become photosynthetic or even change colors. Alternatively, there may be features of the regulation or expression of the genes that is facilitated by such grouping.

R. capsulatus contains three endogenous plasmids, which have been measured by sedimentation analysis to be approximately 42,100 , and $114 \mathrm{~kb}$ in size (Hu and Marrs 1979). The latter two of these are large enough to contain the photosynthesis gene cluster. In order to determine the minimal size DNA fragment upon which the cluster resides, I performed a simple series of experiments. In brief, the work involved embedding the cells in agarose, enzymatically degrading all cellular protein and RNA, and digesting the DNA with one of several restriction enzymes that cut $R$. capsulatus DNA infrequently. The resulting fragments were then separated by pulsed field gel 
electrophoresis on a CHEF device built in the Department of Chemistry using the designs of Chu, Vollrath, and Davis (Chu, Volingth, and Davis 1986b). The methodologies for lysing $R$. capsulatus cells and the gel conditions used are given in the legend to fig 1.10. The most useful enzyme was found to be Xbal. This enzyme cut the chromosome into 12 fragments, ranging in size from 20 to greater than $1000 \mathrm{~kb}$ (fig 1.10). The second largest of these bands, at approximately $510 \mathrm{~kb}$, was the one that contained the PS gene cluster, as shown by Southern hybridization. Similar experiments using Scal and KpnI showed that the PS gene cluster resides on restriction fragments that are 475 and $275 \mathrm{~kb}$, respectively, for the two enzymes. Since each of these is much larger than any of the plasmids detected in the earlier studies, the photosynthesis gene cluster can be assumed to be located not on any of the three plasmids, but rather on the $R$. capsulatus chromosome itself.

The complete genome of the closely related $R$. sphaeroides has been mapped to low resolution with several infrequently-cutting restriction enzymes. Unlike E. coli, which contains one $4700 \mathrm{~kb}$ chromosome (Kohara, Akiyama, and Isono 1987, Riley and Krawiec 1987) and a 100 kb F-factor plasmid (Willets and Skurray 1987), $R$. sphaeroides contains seven circular pieces of DNA for a total genome size of $4400 \pm 120 \mathrm{~kb}$. Two of these have been designated as chromosomes (3046 $\pm 95 \mathrm{~kb}$ and $914 \pm 17 \mathrm{~kb}$ ) on the basis of the presence of essential genes (such as rRNA operons) on each, while the five plasmids are 42 to $113.6 \mathrm{~kb}$ in size, for a total of $450 \mathrm{~kb}$ (Suwanto and Kaplan 1989a, Suwanto and Kaplan 1989b). The R. sphaeroides PS gene cluster lies on the larger of the two chromosomes. There has, to date, been no complete physical or genetic map of the $K$. capsulatus chromosome. It is therefore impossible to tell whether $R$. capsulatus contains very large fragments beyond 

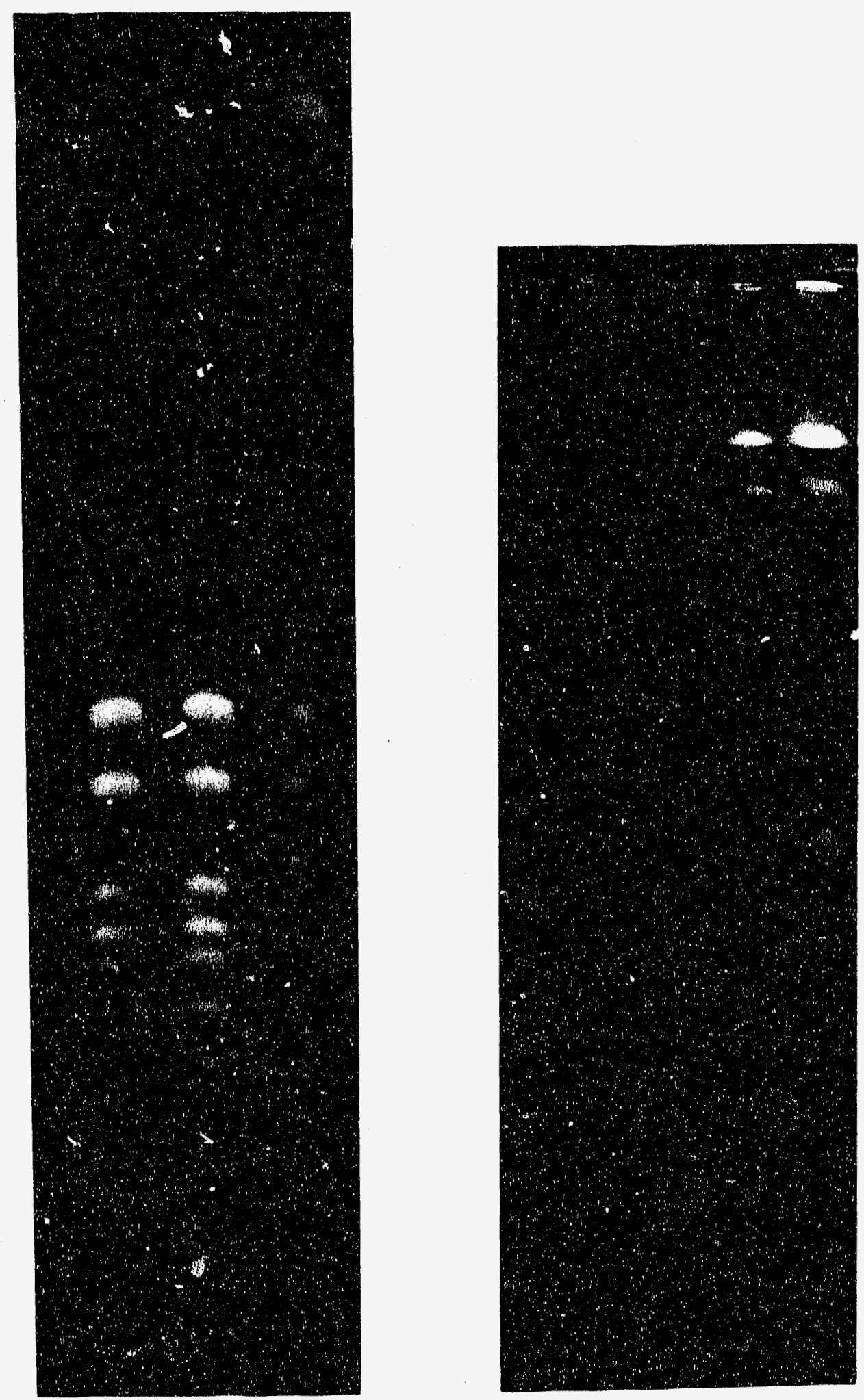

XBB $\quad 926-4987$ 
Fig. 1.10 Pulsed field gels of digested $R$. capsulatus genomic DNA. Left panel: $R$. capsulatus DNA digested with Xbal (lanes $1 /$ and 2 ) and a ladder of concatemers of bacteriophage $\lambda$ DNA. Right panel: $R$. capsulatus DNA digested with KpnI (lanes 1 and 2) or AsnI (lanes 4 and 5), Lane 3 is the $\lambda$ DNA standard. Each band in the $\lambda$ DNA lanes is $48.5 \mathrm{~kb}$ larger than the one below it. Both gels were $1 \%$ agarose in $1 / 4 \mathrm{X} \mathrm{TBE}$ and were run at $14^{\circ} \mathrm{C}$ for $36 \mathrm{hrs}$ at $5.8 \mathrm{~V} / \mathrm{cm}$.

Switching time was 40 seconds for the gel on the left and 30 seconds for the gel on the right. Identification of the bands containing the photosyathesis gene cluster was done by Southern hybridization using radioactive probe containing the BamC fragment of the cluster (not shown).

Preparation of DNA was as follows: $R$. capsulatus cells were grown to log phase, harvested, and washed twice with ice cold $T_{10} E_{100}$ buffer $(10 \mathrm{mM}$ Tris, pH 8.0, $100 \mathrm{mM}$ EDTA). The slurry was warmed to $37^{\circ} \mathrm{C}$ before mixing with an equal volume of 1.69 low-melting temperature agarose (in water) at $42^{\circ} \mathrm{C}$. The suspension was quickly aliquoted into $80 \mu /$ molds and allowed to solidify on ice for about 10 minutes. The "plugs" were pushed out of their molds and into Iysis solution (100 mM NaCl, $10 \mathrm{mM}$ Tris, pH 8.0, $100 \mathrm{mM}$ EDTA), where they were equillibrated for 15 minutes before the addition of $\mathrm{T} 4$ lysozyme to a final concentration of $2 \mathrm{mg} / \mathrm{ml}$. This was incubated with gentle shaking at $37^{\circ} \mathrm{C}$ for 4 hour. The lysis solution was decanted and replaced with ESP solution (100 $\mathrm{mM}$ EDTA, $1 \%$ SDS, $1 \mathrm{mg} / \mathrm{ml}$ proteinase $\mathrm{K}$ ), in which it was incubated for 3 days at $50^{\circ} \mathrm{C}$. The proteinase $\mathrm{K}$ was inactivated by washing the plugs 3 times at 30 minutes each with $\mathrm{T}_{10} \mathrm{E}_{100}$ containing $1 \mathrm{mM}$ phenylethylsulfonyl fluoride (PMSF). The PMSF was then removed by two washes in $\mathrm{T}_{10} \mathrm{E}_{100}$ alone. For overnight digestions, plugs were preequillibrated in the appropriate restriction buffer for 30 minutes before addition of enzyme. 
the limit of resolution of the sedimentation analysis, i.e., whether it shares its cousin's trait of possessing two chromosomes.

In 1988, just as my own efforts at genome mapping were beginning, it was reported that a complete set of ordered cosmid clones spanning all of the R. capsulatus genome had been assembled. Such a set would have contained both the information of a restriction map and the utility of defined, isolated fragments of DNA amenable to further manipulation. I redirected my work away from genomic mapping so as not to waste time competing with work that was already done. Nearly a year later, it became apparent that the ordered set of cosmids was neither ordered nor comprehensivd, but had instead become uselessly "scrambled" during its construction. There have been other occasions when my own work in progress was rumored to have been finished or nearly finished in other labs, but since that earlier experience I have learned not to redirect my own work on the basis of such rumors.

The photosynthesis gene cluster and organization of the thesis.

In a heroic effort spanning several years, Marie Alberti in this laboratory determined the sequence of all 45,959 nucleotides of the photosynthesis gene cluster from Rhodobacter capsulatus. All of the sequence data presented in this thesis were generated by hei: This achievement has propelled work in $R$. capsulatus well ahead of that being done in other organisms. The reaction center genes ( $p u f L$ and $p u f M$ ) have been sequenced from a number of organisms, and a small number of crt genes have recently been obtained from some cyanobacteria, some non-photosynthetic bacteria, and plants, but there are very few.isolated or sequenced $\mathrm{Chl}$ - or $\mathrm{BChl}$ synthesizing genes for any organism other than $R$. capsulatus. The genetic 


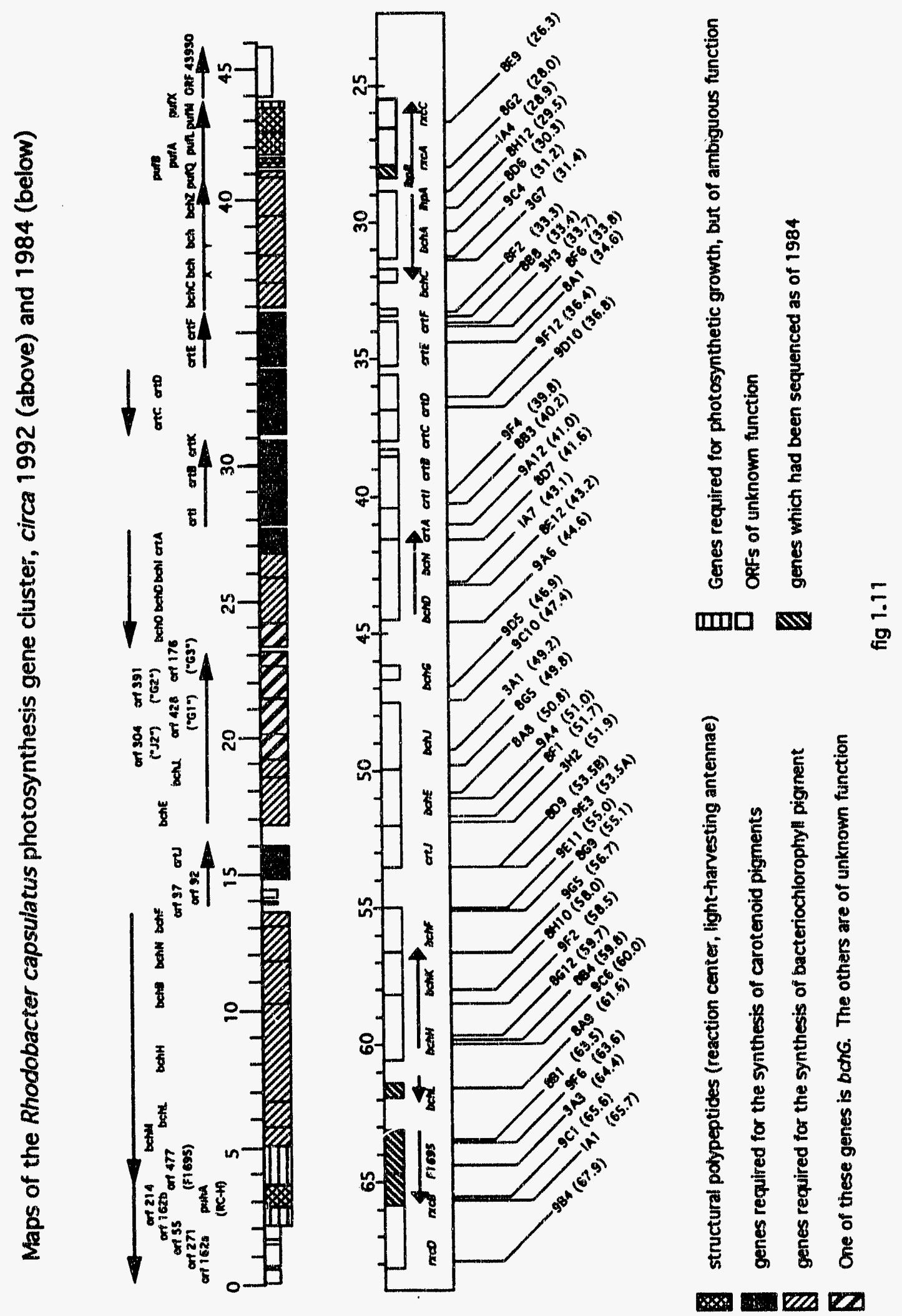


map of the photosynthesis genes of $R$. capsulatus as we currently understand it is given in fig 1.11 .

In this thesis, I detail my efforts to understand several of the genes involved in the production of $\mathrm{BChl}$, which comprise over half of the total gene cluster (55\%). The bch genes have provided numerous surprises. Three consecutive genes (bchXYZ) whose expression is tightly linked had been masquerading as one large gene $(b c h A)$. Delineating their existence as separate entities was the motivation for the work presented in chapter 2 . At the same time, however, it became obvious that the gene products of bchX and of another gene, $b c h L$, (collectively refered to as $\mathrm{Chl} F e$ proteins) are mechanistically and evolutionarily related to each other and to a protein involved in sequestering atmospheric nitrogen out of the air and converting it to a usable form. The implications of these results are also described in Chapter 2. Three genes near the opposite end of the PS gene cluster had been previously mapped in the wrong order and assigned the wrong functions, and the error had been propagated into the subsequent work of several labs. Determining the correct function of these genes, which turn out to be related to the bchXYZ genes, is at the heart of chapter 3. The work represented in both chapters 2 and 3 has led to the identification of three plant genes that are used in Chl synthesis, as well as highlighting the evolutionary milestones that led to the invention of chlorophyll- and bacteriochlorophyll-based photosynthesis. The similarities that we find among the various $b c h$ genes has rather strong implications for the evolutionary history of photosynthesis. A model for the evolution of photosynthesis, based largely on the results presented in the preceding chapters; is given in Chapter 4 . Here I present a scenario in which $\mathrm{BChl}$ came into existence before $\mathrm{Chl}$ (which is contrary to 
the currently prevailiñg view that $\mathrm{Chl}$ arose first), and in which both may owe their existence to a duplication of one of the genes required for nitrogen fixation. 
blank page for numbering only 
Chapter 2: Translational coupling in the bchCXYZ operon of Rhodobacter capsulatus and the generality of chlorophyll Fe proteins in chlorophyll and bacteriochlorophyll synthesis.

\begin{abstract}
The bchA locus of Rhodobacter capsulatus codes for the chlorin reductase enzyme in the bacteriochlorophyll (BChl) synthesis pathway. Previous work has suggested that this locus might comprise a single gene. We have sequenced the $b c h A$ locus and found it to contain three coding segments, designated $b c h X, b c h Y$, and $b c h Z$. Each coding segment contains its own translational initiation sequences and obeys excellent codon utilization throughout. When various regions of the bchA locus and flanking sequences were subcloned into an expression vector and expressed in $E$. coli, the three coding segments were all expressed as separate peptides. Furthermore, expression of $b \operatorname{ch} Z$ in this system is shown to be strictly dependent on prior translation of $b c h Y$, demonstrating that the operon is translationally coupled. Taking all these observations together, we propose that $b \operatorname{chX}, b c h Y$, and $b c h Z$ are separate, translationally coupled genes within a $b c h A$ gene cluster. $b c h X$ is homologous (26-32\% identity) with both protochlorophyllide reductase Fe protein $(b c h L)$ and nitrogenase reductase Fe protein (nifH). These similarities are greatest in regions known to bind MgATP and a [4Fe4S] cluster. bchL, and $b c h X$, and their pigment synthesis homologs therefore define a newly recognized class of proteins, the "Chlorophyll Fe proteins." Using the known crystal structure of nifH protein from Azotobacter vinelandii, we assess the significance of the similarities and propose a mechanism for lightindependent protochlorophyllide and chlorophyllide reductions.
\end{abstract}




\section{INTRODUCTION}

Chlorin reductase is the enzyme that converts chlorophylls (Chl) into bacteriochlorophylls (BChl) by reducing ring $B$ of the tetapyrrole (fig 2.1). Four of the five photosynthetic bacterial divisions (Chloroflexaceae, Protecbacteria, Heliobacteriaceae, and Chlorobiaceae) use $\mathrm{BChl}$ in their reaction centers, and so chlorin reductase is expected to be a widely distributed enzyme. Only the cyanobacteria, which use Chla in their reaction centers, do not synthesize BChl. Chlorin reduction is chemically similar to

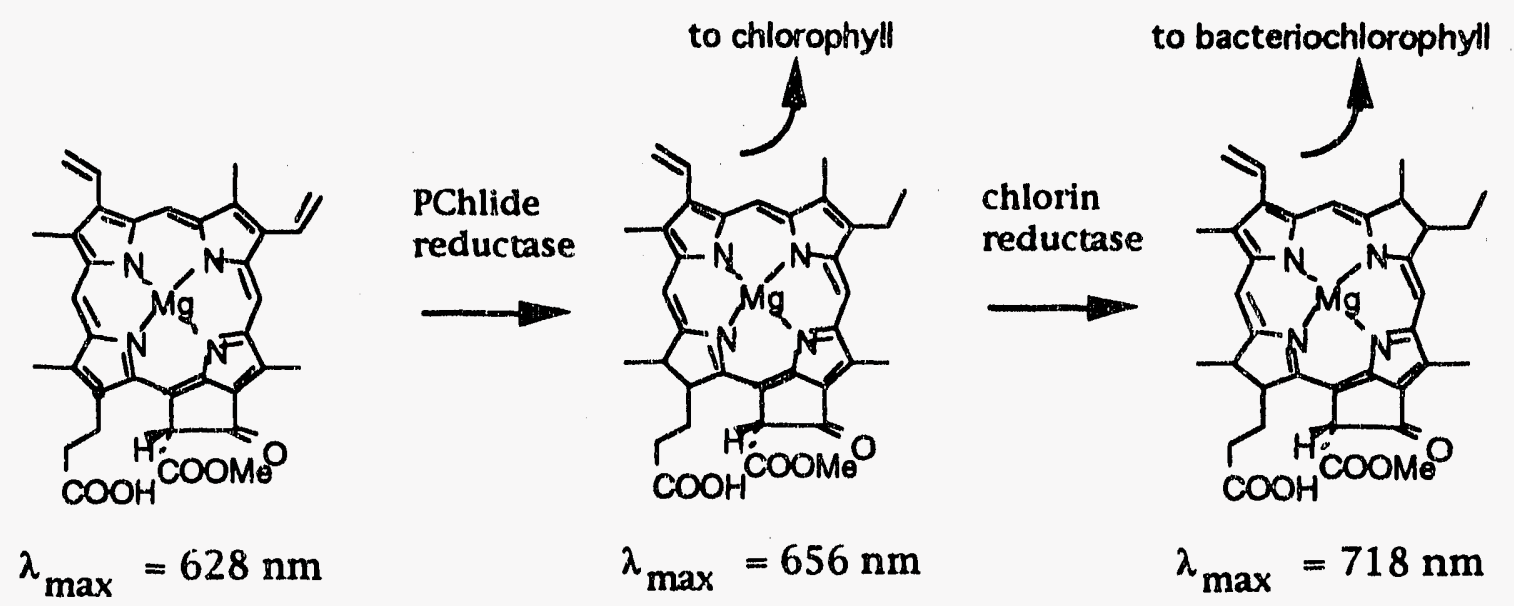

(i)

(ii)

(iii)

fig 2.1 Reduction reactions of $\mathrm{Chl}$ and $\mathrm{BChl}$ synthesis are presented along with the genes coding these steps in $R$. capsulatus. (i) divinyl PChlide $a$.

(ii) Chlide $a$ (iii) 2-desacetyl-2-vinylbacteriochlorophyllide $a$. Either Chlide $a$ or 2-desvinyl-2-( $\alpha)$ hydroxyethyl- chlorophyllide $a$ can serve as substrate for the chlorin reductase (Pudek and Richards 1975). Wavelengths given below the structures are for acetone:methanol (7:2) extracts of mutatiols in $b c h B$, $b c h Z$, and $b c h F$, respectively. 
protochlorophyllide (PChlide) reduction in both the $\mathrm{BChl}$ and the $\mathrm{Chl}$ synthetic pathways. The two enzyme systems might therefore be expected to be similar. However, there are at least two genes required for PChlide reduction, bchB (Yen and Marrs 1976) and bchL (Coomber et al. 1990, Yang and Bauer 1990), and possibly a third, bchN (Burke, Alberti, and Hearst 1992b), whereas only one gene, bchA (Yen and Marrs 1976), is known for the chlorin reductase.

Rhodcbacter capsulaius mutants deficient in chlorin reductase accumulate 2-desvinyl-2-( $\alpha)$ hydroxyethylchlorophyllide $a$ (Richards and Lascelles 1969), but chlorophyllide $a$ can also serve as substrate for the reaction (Pudek and Richards 1975) (fig 2.1). Both compounds absorb maximally at around $660 \mathrm{~nm}$, and this absorbance maximum defines the BchA phenotype (Yen and Marrs 1976). The bchA genetic locus is downstream from $b c h C$ on the same operon, the transcriptional start site of which has been mapped (Wellington and Beatty 1989) to the nucleotide position predicted from the DNA sequence (Armstrong et al. 1989a). The promoter for this operon has been studied in detail (Ma 1992a), and has been found to include several control elements, including a $\sigma^{70}$-like promoter, a palindromic sequence which may bind a repressor, and an AT-rich region. In addition, this operon may be part of a larger unit of gene expression, the crtEF-bchCA-pufQBALMX superoperon (Wellington and Beatty 1989, Wellington and Beatty 1991, Young et al. 1989), in which transcription initiated in each of the two upstream operons continues into the downstream puf operon.

The bchA locus has been thought of traditionally as a single, large gene on the basis of two lines of evidence, although neither excludes the possibility 
of additional chlorin reductase genes within this region: First, all of the mutations in the $4.6 \mathrm{~kb}$ between $b c h C$ and $p u f Q$ give the BchA phenotype. These results, however, would also have been obtained if more than one gene required for chlorin reductase activity is present in the same region. Second, a plasmid carrying the promoter and all but the $3^{\prime}$ terminal 800 b.p. failed to complement three point mutants which all lie in the first half of the $b c h A$ region. This second set of results also cannot distinguish a single gene from polar inactivation of downstream coding elements, which would also have led to failed complementation. The "single $b c h A$ gene" point of view is also in contrast to earlier reports of a smaller " $R$ protein" immediately upstream from pufQ in the closely related bacterium $R$. sphaeroides (Kiley and Kaplan 1988).

As part of our efforts to understand photosynthetic pigment biosynthesis, we have sequenced the bchCA operon of $R$. capsulatus and found it to contain four genes rather than two. We report the sequence here, along with the results of heterologous expression studies of various subsections of this operon in $E$. coli. Our results settle the dispute of "one gene" vs. "multiple genes" for chlorin reductase on the side of the plural, and we further demonstrate that the genes in this operon are translationally coupled. Finally, we discuss the significance of the similarities among the first coding segment of the "bchA cluster" $(b c h X)$ and a subunit of the PChlide reductase $(b c h L)$ and the nitrogenase $\mathrm{Fe}$ protein ( $n$ ifH), in terms of the three-dimensional structure and enzymatic mechanism of PChlide reductase and chlorin reductase. These similarities lead us to describe a new class of proteins, the "Chl Fe proteins," whose properties are predicted to be similar to those of the nitrogenase Fe protein. 


\section{METHODS \& MATERIALS}

materials. Radionucleotides were purchased from Amersham, Notl restriction endonuclease from Toyobo, and other endonucleases from New England Biolabs or Bethesda Research Labs (BRL). DNA ligase was purchased from BRL and Amersham, Klenow from BRL, and Taq DNA Polymerase from Perkin Elmer. Deoxy- and dideoxynucleotide triphosphates were from Pharmacia, DNA molecular weight size standards were from BRL, protein molecular weight size standards were from Sigma. Oligodeoxynucleotides were synthesized by David Koh using phosphoramidite chemistry on an Applied Biosystems model 381 a automated synthesizer.

plasmids and strains. The adjacent $B a m H I$ fragments $J$ and $C$ of the Rprime plasmid pRPS404, which carries the entire photosynthesis gone cluster of $R$. capsulatus, were subcloned separately and together into the BamHI site of pBR325 to form plasmids pFL227, pFL226, and pFL114, respectively. Overlapping M13 subciones in both directions were used in sequencing reactions.

Plasmids pDBX102a, pDBX102b, pDBX102c, and pDBX105. The $4.64 \mathrm{~kb}$ BamHI-Sall fragment of pFL226 was subcloned into pUC19 to form pDBX2. This same fragment was blunt-ended with Klenow and fused with the plasmidencoded phage $\phi 10$ protein in each translational frame at the blunt ended BamHI site of the expression vectors pET3a, pET3b, and pET3c (Studier et al. 1990) to form plasmids $\mathrm{pDBX102a}$, pDBX102b and pDBX102c, respectively. Plasmid pDBX105 was formed by partial Notl digestion of pDBX102c, followed by recircularization. 
Plasmids pDBX21, pDBX123, pDBX124, and pDBX125: Two oligonucleotide primers, X-2 (GCGCTGAGCTCTAAAGAAGGAGATCTACATATGACCGATCTTCCGCAAGCCGAAGG) and Y-1 (CGCCAGAGGCGGTGGGCACGCAAA) were used to amplify a 400 bp fragment from $1 \mathrm{ng}$ of plasmid $\mathrm{pDBX} 2$. Sstl and Ndel restriction sites used in cloning are underlined. Amplification was for 20 cycles of $\left(94^{\circ}, 1\right.$ minute; 550, 2 minutes; 720,3 minutes). Due to competition from the internal structure of the template for binding of primer $X-2$, this amplification proved extremely difficult, and was only achieved when $10 \%$ glycerol was used and 7-deAza dGTP replaced dGTP. This amplification removed the competing hairpin at the $5^{\prime}$ end of bchY but introduced a potentially mutagenic nucleotide into the sequence. The amplified material was therefore diluted $10^{4}$-fold and reamplified using all normal dNTPs before digestion and cloning. Plasmid pDBX2 was passaged through E. coli strain GM2361(dam-) so that the amplified material could replace the SstI to Bcll fragment to form pDBX21. The $3.25 \mathrm{~kb} N$ deI to BstEII fragment from this plasmid replaced the corresponding fragment in pDBX102c to form pDBX123. $\mathrm{pDBX} 123$ was digested with NotI and recircularized to form pDBX124, or the Notl ends were first filled in with the Klenow fragment of DNA Polymerase and recircularized to form pDBX125.

Plasmids pDBX3, pDBX4, and pDBX204: bchX was amplified between primers X-3 (AGAGAGGATCCCATATGACTGACGCACCCAACCTGAAGGGATTT) and X-6 (CCCCTCXAGATCATGCCCGGCCCTCCGTGCAAGCGGCCCGGGCTTTC), which introduces BamHI and Ndel sites at the $5^{\prime}$ end of the gene and an Ybal site at the $3^{\prime}$ end (underlined). The long primers and a slow ramp from $50^{\circ} \mathrm{C}$ to $72^{\circ} \mathrm{C}$ allowed X-6 to compete with the strong secondary structure shown in figure 2.5 . This fragment was cloned between the BamHI and XbaI sites on pUC19 to generate pDBX3. The $2.2 \mathrm{~kb} \mathrm{XmnI} \mathrm{fragment} \mathrm{of} \mathrm{pDBX2} \mathrm{was} \mathrm{replaced} \mathrm{by} \mathrm{the} 1.6 \mathrm{~kb} \mathrm{XmnI}$ 
fragment of pDBX3 to generate pDBX4. The $4.0 \mathrm{~kb} \mathrm{BamHI}$ to Sall fragment of pDBX4 was then cloned into the corresponding sites on the expression vector pUHE21-2 (Stuber and Bujard 1982) to generate pDBX205, in which bchX is fused in frame after the third codon of the vector.

All $E$. coli strains used in this study are common ones that are described in detail elsewhere (Sambrook, Fritsch, and Maniatis 1989). pFL constructs were maintained in HB101. JM103 was used for growth and propagation of M13 phage constructs. pDBX constructs were initially cloned into DH5 $\alpha$. Those used in expression studies were transfered to BL21(DE3) lysogen (Studier et al. 1990), which contains phage T7 polymerase under control of the lac repressor. The exception was pDBX204, which was cloned and maintained in D1210, a lacl $\mathrm{Q}$ derivative of HB101. Growth of E. coli strains was in LC medium (Marrs 1981) or on LC plates containing $1.5 \%$ agar. Ampicillin was used at $100 \mu \mathrm{g} / \mathrm{ml}$ except where noted below.

Induction of expressed proteins with IPTG was performed essentially as described previously (Studier et al. 1990). All of the constructs used in this study were assayed for plasmid stability (Studier et al. 1990). All of the constructs are stable in their respective host strains, and none of the expressed products is toxic to the host at the low level of constitutive expression while under lac repression. Total proteins were assayed by discontinuous SDS-PAGE. Transfer to Immobilon polyvinylidene difluoride (PVDF) membranes (Millipore) was as per the manufacturer's recommendations. Bands that were to be assayed by $\mathrm{N}$-terminal sequencing or by total amino acid composition analysis were excised and rinsed extensively with water to remove traces of glycine used in the transfer buffer. Protein 
sequencing and amino acid composition analysis were performed at the Protein Structure Laboratory of the University of California at Davis.

Chromosomal sequencing. Serial dilutions of lysate from $R$. capsulatus wild-type strain SB1003 were subjected to PCR amplification between primers X-1 (AGGAAACCCGGGTCGTCGATCTGG) and X-6 using the same reaction buffer as above. Five cycles of $\left(94^{\circ}, 1\right.$ minute; $50^{\circ}, 4$ minutes, 3 minute ramp up to $72^{\circ}$, and 90 seconds at $72^{\circ}$ ) were followed by twenty cycles of the same profile without the ramp. Optimal amplification was observed when approximately $10^{7}$ cells were used, corresponding to roughly $100 \mathrm{ng}$ of total DNA. The desired fragment was excised from a $1.6 \%$ agarose gel and reamplified through fifty cycles of asymmetric PCR with a $55^{\circ} \mathrm{C}$ minimum temperature and a 100:1 exces.; of each primer in separate reactions. Single-stranded DNA was extracted twice with organic solvent and precipitated in ethanol. The resuspended pellet was then sequenced from each end without cloning, using the dideoxy chainterminating sequencing reaction (Sanger, Nicklen, and Coulson 1977). The sequence of the amplified fragment confirmed was the same as that of the M13 subclone from which the sequence had been originally determined.

Computationsl sequence nalysis. RNA folding analysis was done using the Zuker program (Jaeger, Turner, and Zuker 1989) with the assistance of Dr. Joseph Monforte. Features that were present in most or all of the foldings within $10 \%$ of the lowest energy structure were considered significant. Database searches were performed on a cluster of Sun computers in the Depariment of Molecular and Cell Biology at UC Berkeley using the fastdb program (Brutlag et al. 1990) of the Intelligenetics suite of sequencing 
Mract...

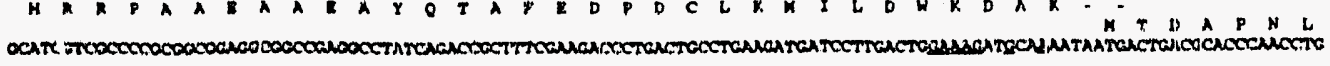

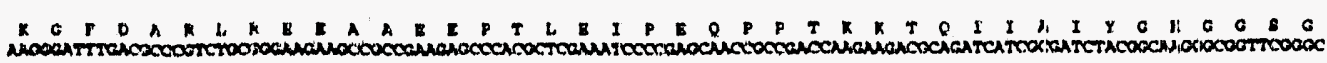

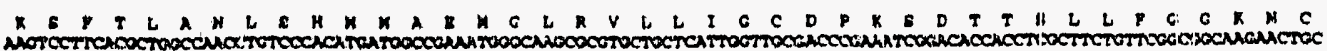

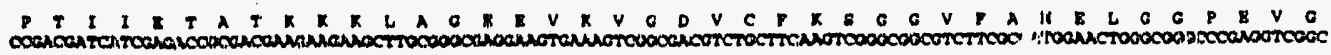

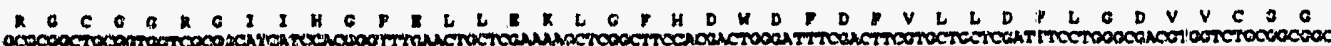

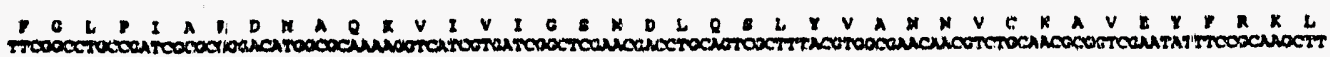

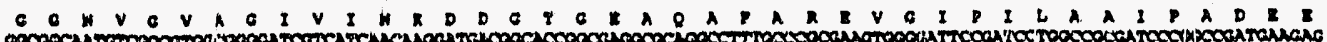
0000004 N

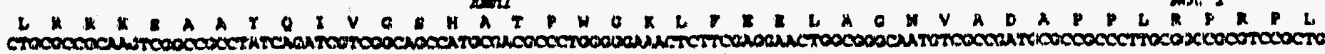

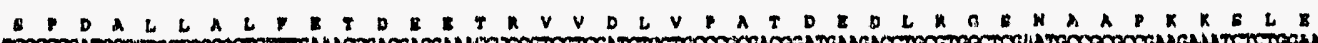

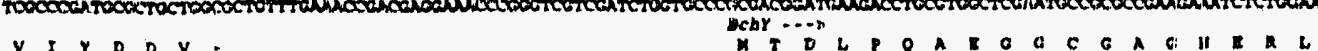

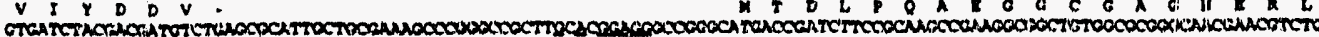

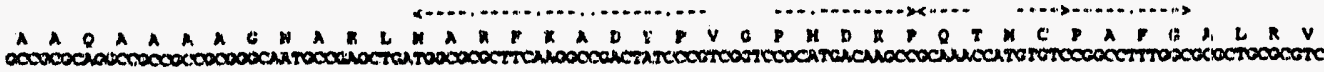

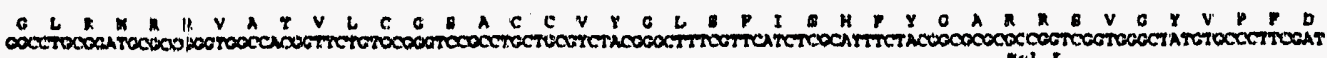

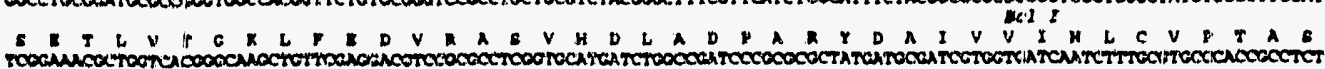

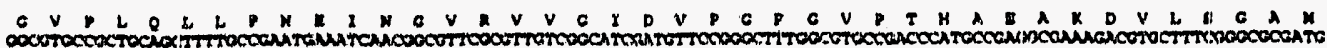

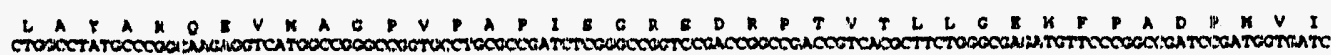

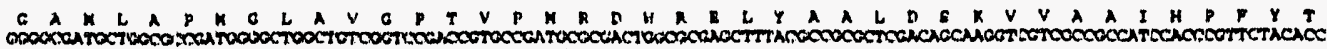

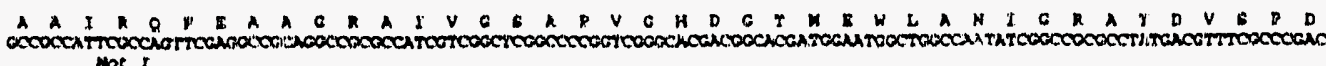

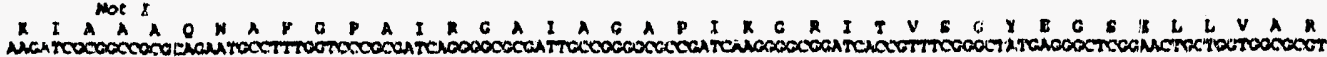

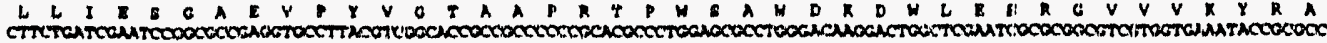

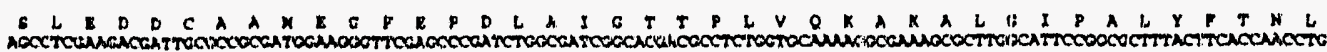

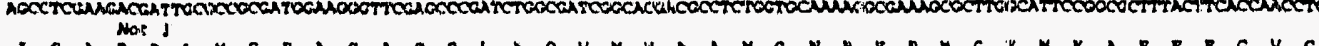

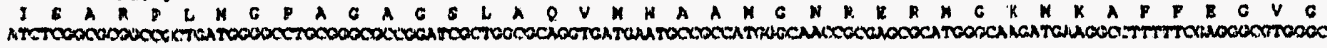

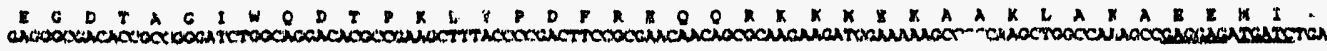

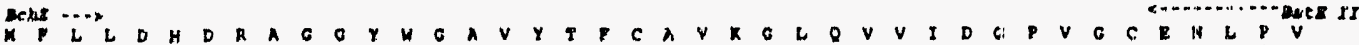

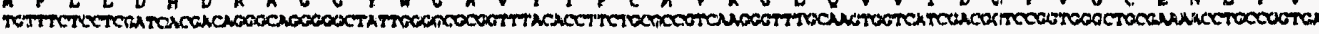

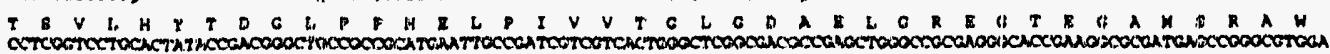

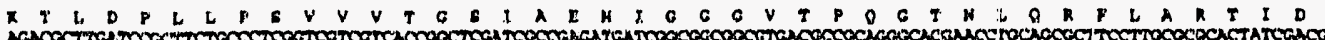

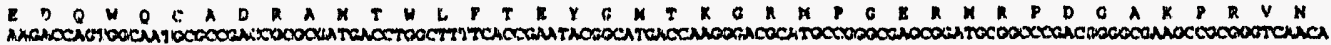

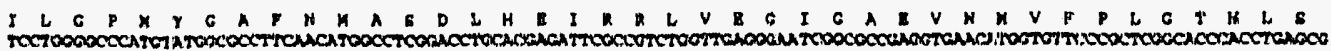

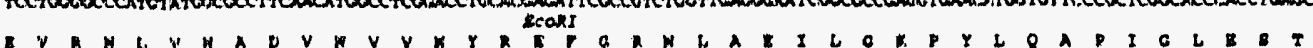

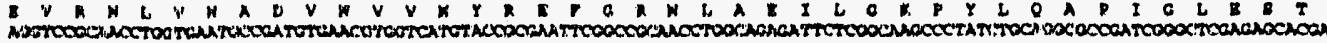

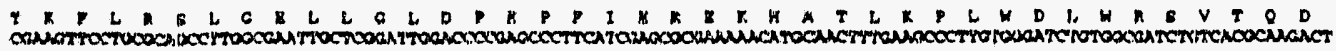

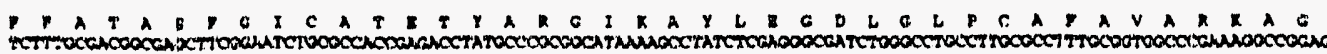

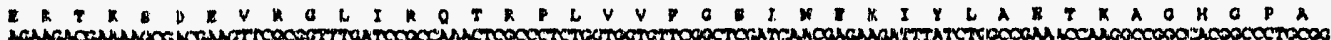

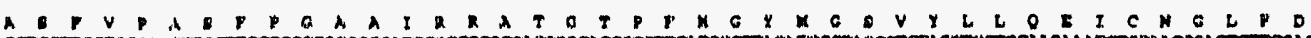

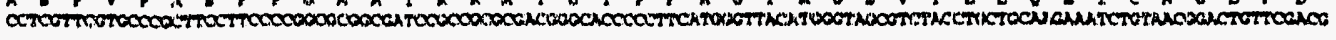

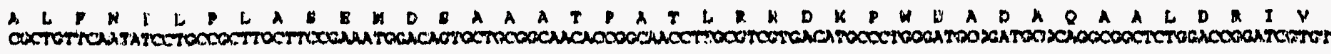

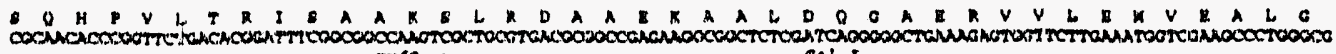

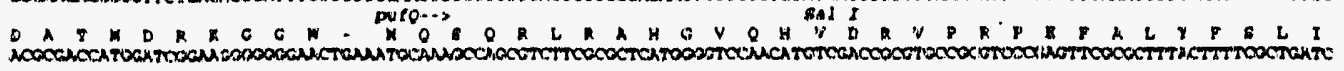

3688

37006

37220

227
37280

267
37366

200

247
37800

267

37720

307
37848

28
37968

3e0et

38208

130
30320

secise

229
38568

259
38600

299
38608

339
31928

379
39048

30160

459
39260

39000

10
39528

39618

220
39768

260
39040

200

40000

210
.0220

220
0248

320

.0360
360

.0680

$60 n$
40600

110
61728

0100
.0048

29
$\quad 0068$ 
fig 2.2. DNA and deduced amino acid sequences of the three subunits (bchXYZ) of the chlorin reductase (bchA) of Rhodobacter capsulatus. Potential ribosome binding sites are underlined, and potential stem loop structures described in the text are indicated by arrows. Restriction sites used in this study are indicated above the sequence. Sequence numberings (with respect. to the entire $R$. capsulatus photosynthesis gene cluster numberings, EMBO accession number $\mathrm{Z} 11165)$, are given in the right-hand margin. 
software. Databanks searched were GenBank 69 (Dec. 15, 1990), PIR 29 (Sept. 30, 1990), SWISS-PROT 19.0 (Aug. 1990), and EMBL 28 (Jan. 1991). The DNA sequences presented in this report have been deposited in the EMBL DNA sequence databank under accession number $\mathrm{Z} 11165$.

\section{RESULTS}

The three coding segments of $b c h A$.

The bchA locus contains three genes, bchX,bchY, and $b c h Z$. We have determined the nucleotide sequence of the EcoRI-H and $\mathrm{Q}$ fragments of Rhodobacter capsulatus (fig 2.2). Our bchC (formerly "ORFJ" (Armstrong et al. 1989a)) sequence is identical to that of Wellington and Beatty (1989). The bchA region contains three sequential coding segments (fig 2.2), which we designate $b c h X, b c h Y$, and $b c h Z$. Each coding segment shows correct codon usage for $R$. capsulatus and is preceded by a ribosome binding site at appropriate spacing. These three genes had not been resolved in previous genetic complementation experiments with this region (Young et al. 1989). We therefore chose a different approach to test whether $b c h X, b c h Y$, and $b c h Z$ can be expressed as individual polypeptides. Various segments of the bchCXYZ operon were subcloned downstream of a promoter for the phage T7 RNA polymerase (Studier et al. 1990). Vector-initiated translation in these experiments is driven from the efficient RBS of phage gene 10, the major capsid protein of phage T7. Expression from each T7 construct was assayed in E. coli strain BL21(DE3) (Studier et al. 1990), which contains a chromosomal copy of the gene for T7 RNA polymerase under lac repression. The plasmids used in this study are shown in figure 2.3 and are described in methods. 
Plasmid pDBX102c contains all of bchXYZ, along with the C-terminal 196 amino acids of $b c h c$ fused in frame with the first 13 amino acids of the vector. encoded phage protein $\phi 10$. Translation initiated from the vector should terminate at the $b c h C-b \operatorname{chX}$ juncture, allowing expression of the remainder of the operon to be directed by $R$. capsulatus signals. Induction of BL21(DE3) cultures containing pDBX102c yields two newly synthesized peptides with $\mathrm{MW}_{\mathrm{r}}$ of 24 and $41.5 \mathrm{kd}$, respectively (fig $2.4 \mathrm{a}$, lane 2 ). The identity of the peptide in each band was established by $\mathrm{N}$-terminal amino acid sequencing and total amino acid composition analysis. Both measures confirmed the faster-moving species as the $\phi 10-b c h C$ fusion peptide and the slower one as $b c h X$. Other plasmid constructs (pDBX102a \& $\mathrm{pDBX102b}$ ) with out of frame fusions between $\$ 10$ and $b c h C$ produced no newly synthesized products (data not shown).

There is no detectable expression of $b c h Y$ or $b c h Z$ from $\mathrm{pDBX102c}$ or pDBX204, even though both are able to direct high-level expression of fulllength $b c h X$. Similar results were obtained when $b c h X, b c h Y$, and $b \operatorname{ch} Z$ expression was driven by $E$. coli RNA polymerase under the control of the inducible $\mathrm{P}_{\mathrm{A} 1}$ promoter on pDBX204 (fig 2.4a, lane 4). We therefore constructed pDBX123 in order to determine whether $b c h Y$ and $b c h Z$ are capable of being expressed in $E$. coli. The AUG start of $b c h Y$ in pDBX123 is placed seven nucleotides from the vector RBS, so that translational initiation of $b \operatorname{ch} Y$ is driven directly from the vector signals. The mRNA sequence near the RBS of $b c h Y$ in the resulting construct cannot form the very stable hairpin available to the wildtype sequence (see below and fig. 2.5). Induction of BL21(DE3) cultures containing pDBX123 leads to the synthesis of two new peptides of approximately equal intensities with $\mathrm{MW}_{\mathrm{r}}$ of 51 and $53 \mathrm{kd}$, (fig 2.4a, lane 3). The predicted molecular weights of the $b c h Y$ and $b c h Z$ gene products are 52.6 


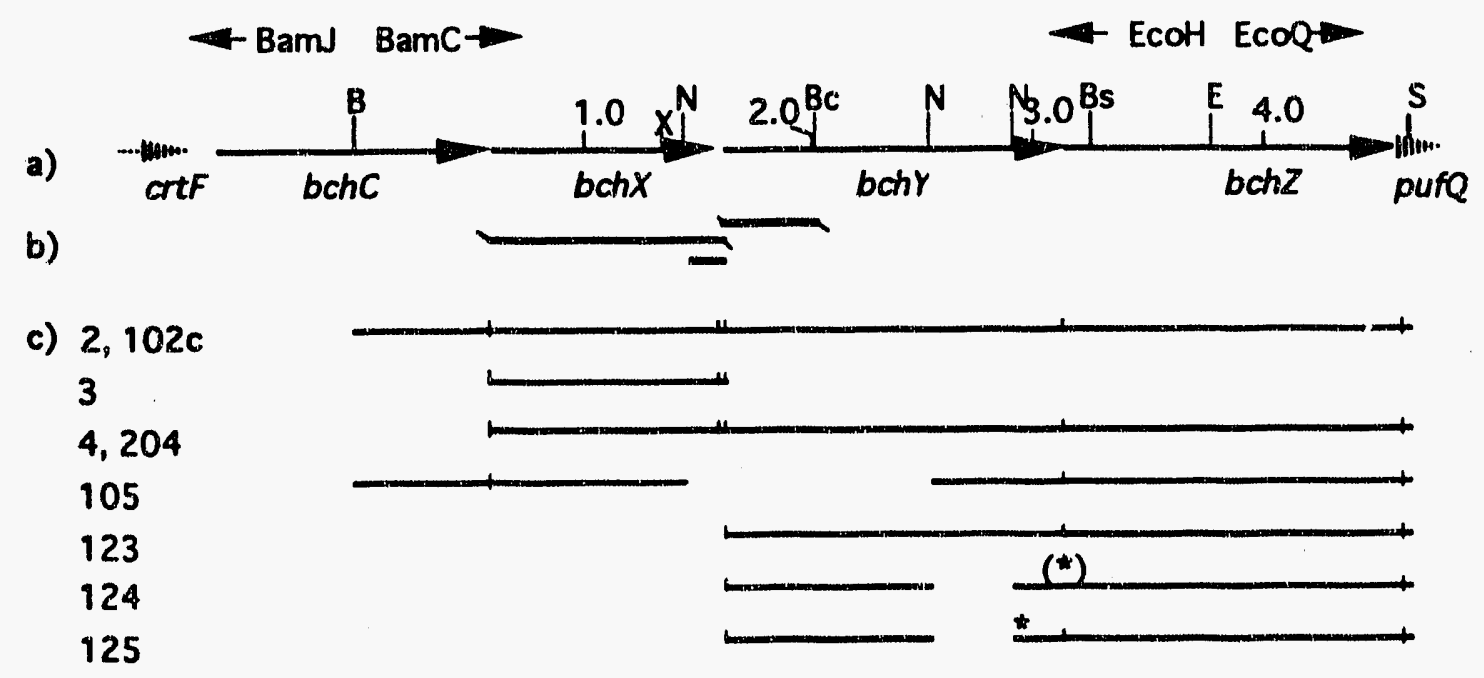

fig 2.3. Subclones of the bchCXYZ operon used in this study. a) Gene map, showing the locations of restriction sites used in cloning: $B=$ $B a m H I, \mathrm{Bc}=B c l I, \mathrm{Bs}=B s t E I I, \mathrm{E}=E c o R I, \mathrm{~N}=$ NotI $, \mathrm{S}=S a l I, \mathrm{X}=\mathrm{XmnI}$. Numbers refer to distance in $\mathrm{kb}$ from the BamHI site at the $5^{\prime}$ end of the BamC fragment at position 36280 . b) Location of the two PCR fragments used in cloning (top and middle) and the fragment used in chromosomal sequencing (bottom). Tails indicate mismatches to the $R$. capsulatus sequence where restriction sites were incorporated into the PCR product. c) Schematic representation of the subclones used in this study. pDBX plasmid numbers are given on the left. Wild-type gene boundaries are indicated by a vertical hash mark (1). Stop codons that are encountered prematurely or are not seen by the ribosome because of frameshifts introduced during cloning are indicated as "*" and "(*)", respectively. 
a

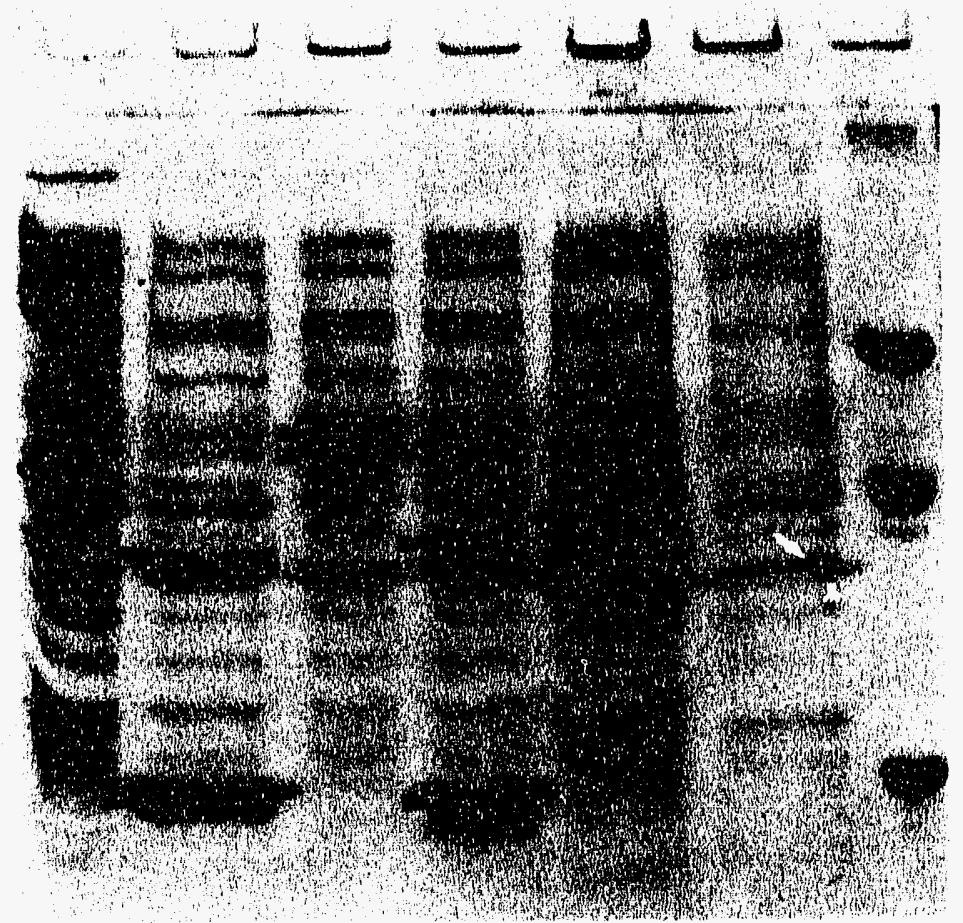

b

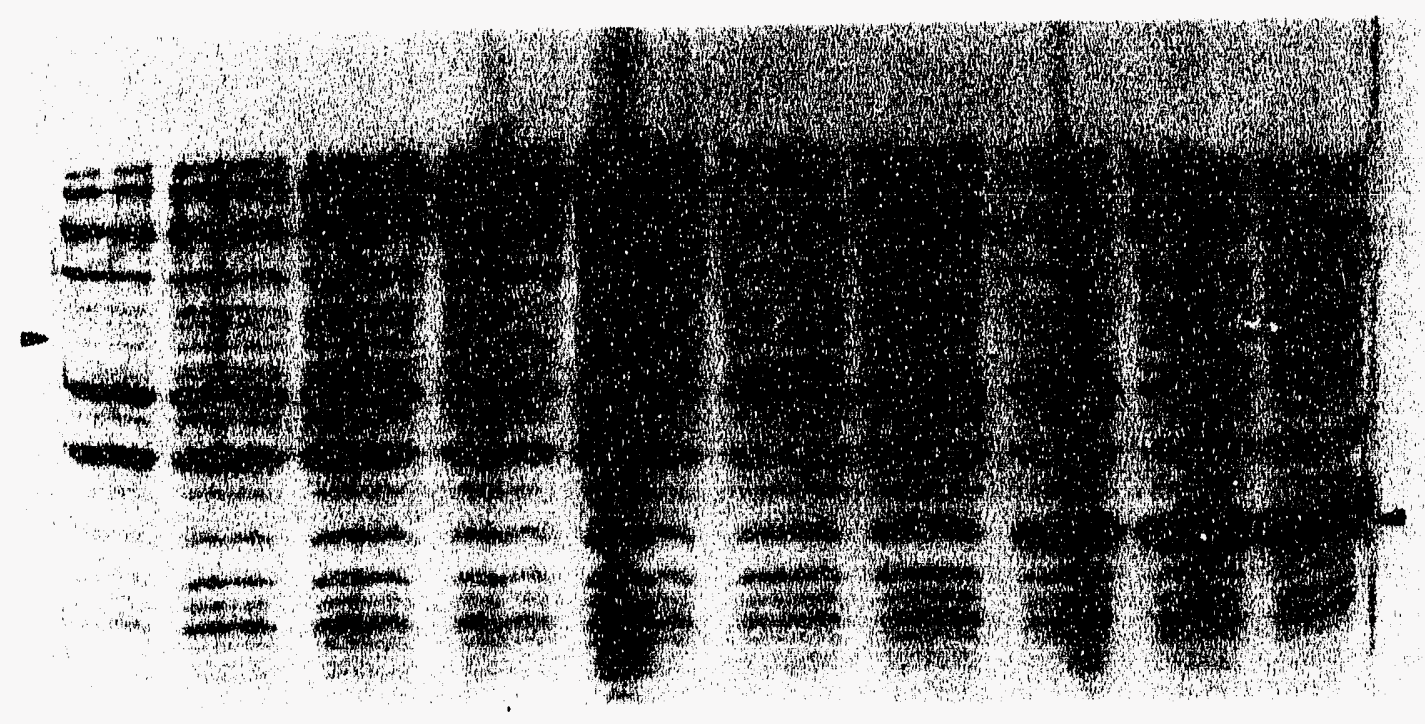

XBB $924-3094 B$ 
fig 2.4. PAGE gel of indur ad proteln. a) IPTG-induced BL21(DE3) cultures carrying overexpression constructs pDBX102c (lane 2), pDBX123 (lane 3), and pDBX105 (lane 4), and an induced D1210 culture carrying pDBX204 (lane 5). Lanes 1 and 6 contain uninduced BL21(DE3) and D1210 cultures, respectively. Molecular weight markers are in lane 7 . Induced products are indicated by triangles. b) Time-course of protein from induced BL21(DE3) cultures carrying overexpression constructs pDBX124 (lanes 1-5), and pDBX125 (lanes 6-10). Cultures were uninduced (lanes 1, 6) or induced with IPTG for 10 minutes (lanes 2, 7), 30 minutes (lanes 3, 8), 90 minutes (lanes 4, 9), or 240 minutes (lanes 5, 10). The triangles to the right of each set of five lanes indicates the overexpressed band. The triangle at the left indicates the expected position of induced bchz gene product. 
and $53.2 \mathrm{kd}$, respectively. N-terminal sequencing of both of these bands excised from PVDF membranes confirmed the identity of the upper band as the product of $b c h Y$ and of the lower as that of bchZ. Our conclusion, based on the results of expression from plasmids $\mathrm{pDBX} 102 \mathrm{c}$ and $\mathrm{pDBX} 123$, is that each of the three coding segments, $b \operatorname{chX}, b c h Y$, and $b \operatorname{ch} Z$ can be expressed as individual polypeptides.

\section{$b c h Y$ and $b c h z$ are translationally coupled.}

Two aspects of the sequence suggest that the region may be translationally coupled. First, strong potential stern-loop structures (fig 2.5) are predicted to form which occlude the RBS for bchY $(-31.7 \mathrm{kcals} / \mathrm{mol})$ and $b c h Z(-9.9 \mathrm{kcais} / \mathrm{mol})$. Continuous translation of a single template may be required in order for the ribosome to be able to find where to begin translating these two genes (see discussion). Second, translational stops and starts overlap between $b c h C$ and $b c h X$ and between $b c h Y$ and $b c h Z$. This feature has frequently been observed in translationally coupled genes, particularly where the RBS of the downstream gene is weak or can potentially be hidden by a folded message (Das and Yanofsky 1989, Ivey-Hoyle and Steege 1989).

Plasmid pDBX105 is identical to pDBX102c except that the 1101 nucleotide NotI fragment from nucleotides 37715 to 38816 is deleted to create an in-frame $b c h X-b c h y$ fusion. The $38.3 \mathrm{kd}$ fusion peptide predicted to be expressed from pDBX105 migrates instead at $43 \mathrm{kd}$ (fig. 2.4a, lane 4), while the two other peptides expressed from this construct comigrate with the $\phi 10-b c h C$ fusion peptide and with bchZ. N-terminal sequencing of the $43 \mathrm{kd}$ peptide confirmed its identity as the $b c h X-Y$ fusion. The ability of this construct to direct $b c h Z$ 


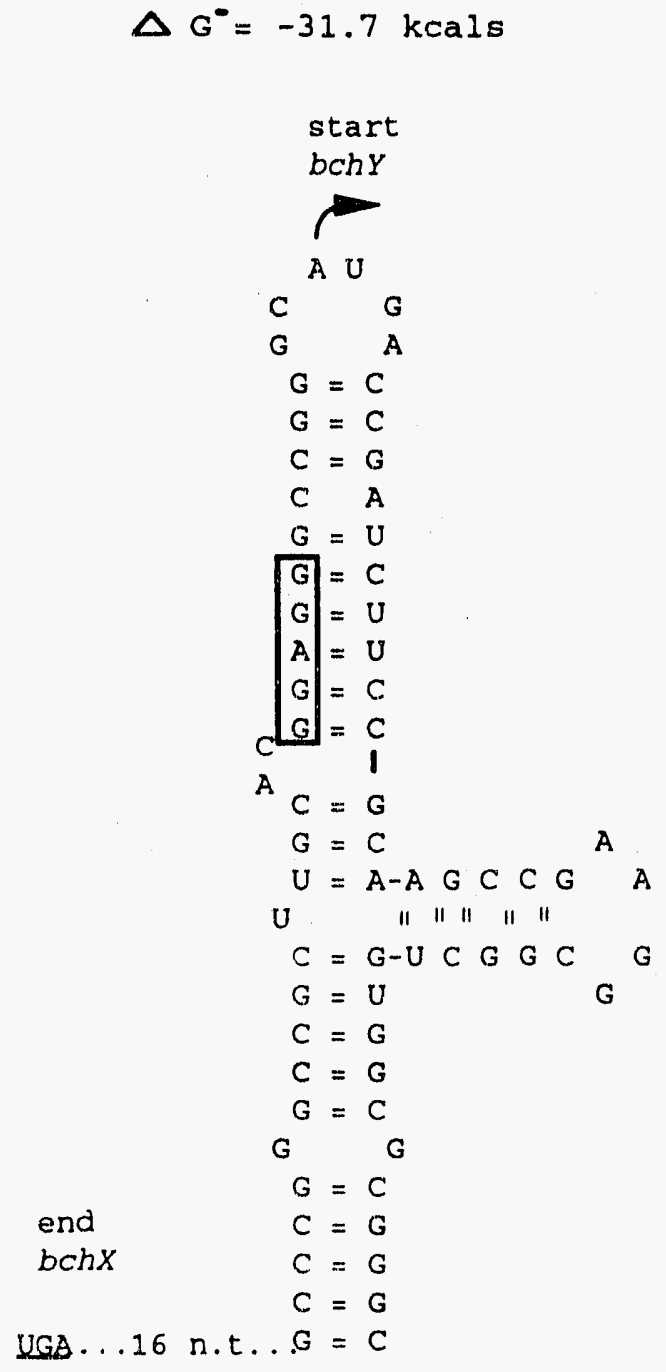

bchr-->bchY
$\Delta G^{\circ}=-12.2 \mathrm{kcals}$

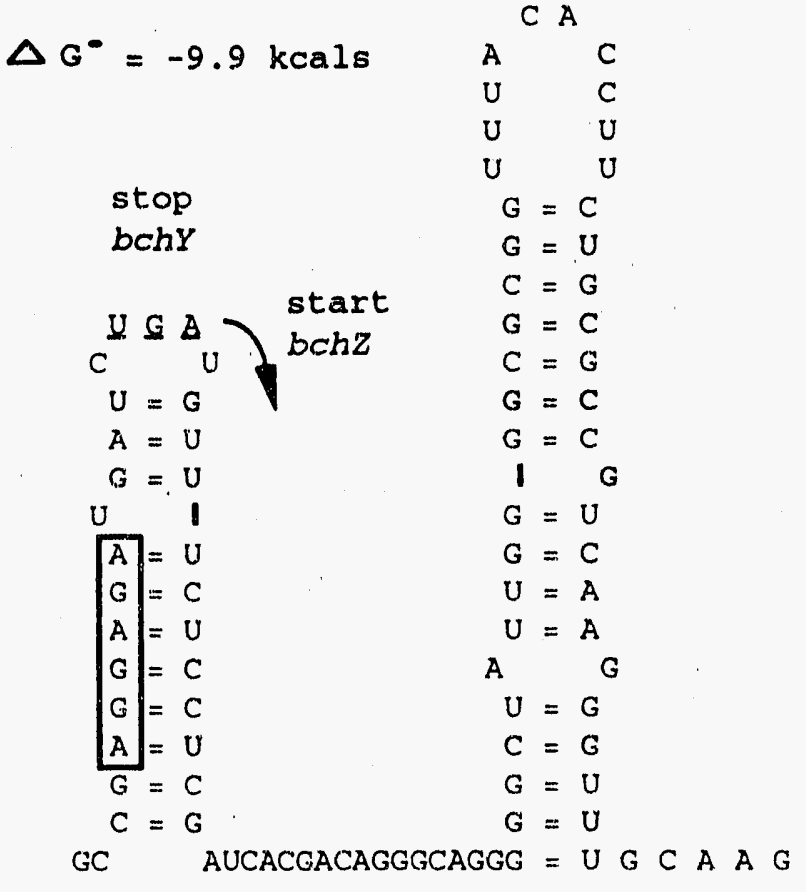

bchY-->bchz

Fig. 2.5 Potential mRNA secondary structure in the vicinity of the initiation signals for $b c h Y$ and $b c h Z$. Stop and start codons are indicated, and potential ribosome binding sequences are boxed. 
expression also demonstrates that some sequence within the deleted fragment between $b c h X$ and $b c h Y$ controls $b c h Z$ initiation.

The last set of constructs were designed to test whether the site of $b c h Y$ termination would influence the efficiency of bchZ expression. The frameshift in pDBX124 created by deletion of the NotI fragment internal to $b c h Y$ in pDBX123 fuses the first half of $b c h Y, a 77$ amino acid missense linker in the same frame as bchZ, and all of bchZ into a $93 \mathrm{kd}$ peptide. A new peptide in this range is observed upon IPTG induction, but no wild type bchZ product accumulates (fig 2.4b, lanes 2-5). The reading frame of $b c h Y$ was again altered by cutting the unique NotI site of pDBX124, filling in the ends with Klenow fragment, and rejoining to produce pDBX125. The $31.9 \mathrm{kd}$ truncated bchY peptide terminates at the UGA immediately after the NotI site, 221 nucleotides before the beginning of bchZ. Induction of BL21(DE3) cultures containing pDBX125 results in production of a new peptide of approximately $30 \mathrm{kd}$, which we take to be the truncated bch $Y$ gene product (fig. 2.4b, lanes 7-10). Again, no significant amount of $b c h Z$ synthesis is observed. We therefore conclude that $b c h Z$ synthesis is dependent on prior translation of $b c h Y$ and termination at its normal stop codon; in other words, the two genes are translationally coupled.

$b c h X$ and $b c h Z$ share amino acid identities with other proteins. Comparisons of the amino acid sequences of $b \operatorname{chX}, b c h Y$, and $b c h Z$ with protein data banks and with translations of nucleic acid data banks scored matches for $b c h X$ and $b c h Z$. The amino acid sequence of $b c h Z$ aligns very well with the bchZ sequence of Rhodobacter sphaeroides (Hunter et al. 1991) and with a partial sequences from Rhodopseudomonas viridis (Wiessner, Dunger, 


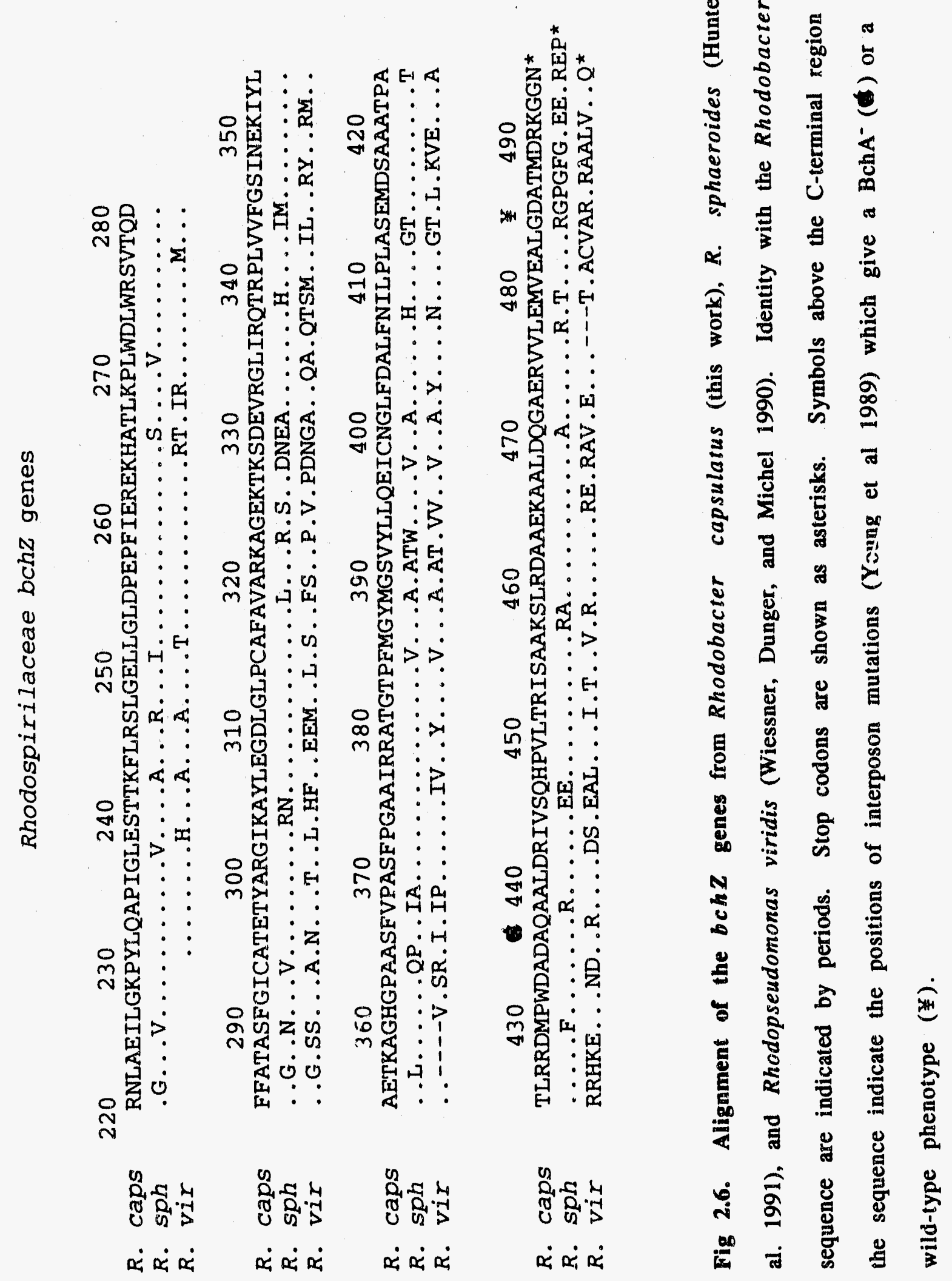




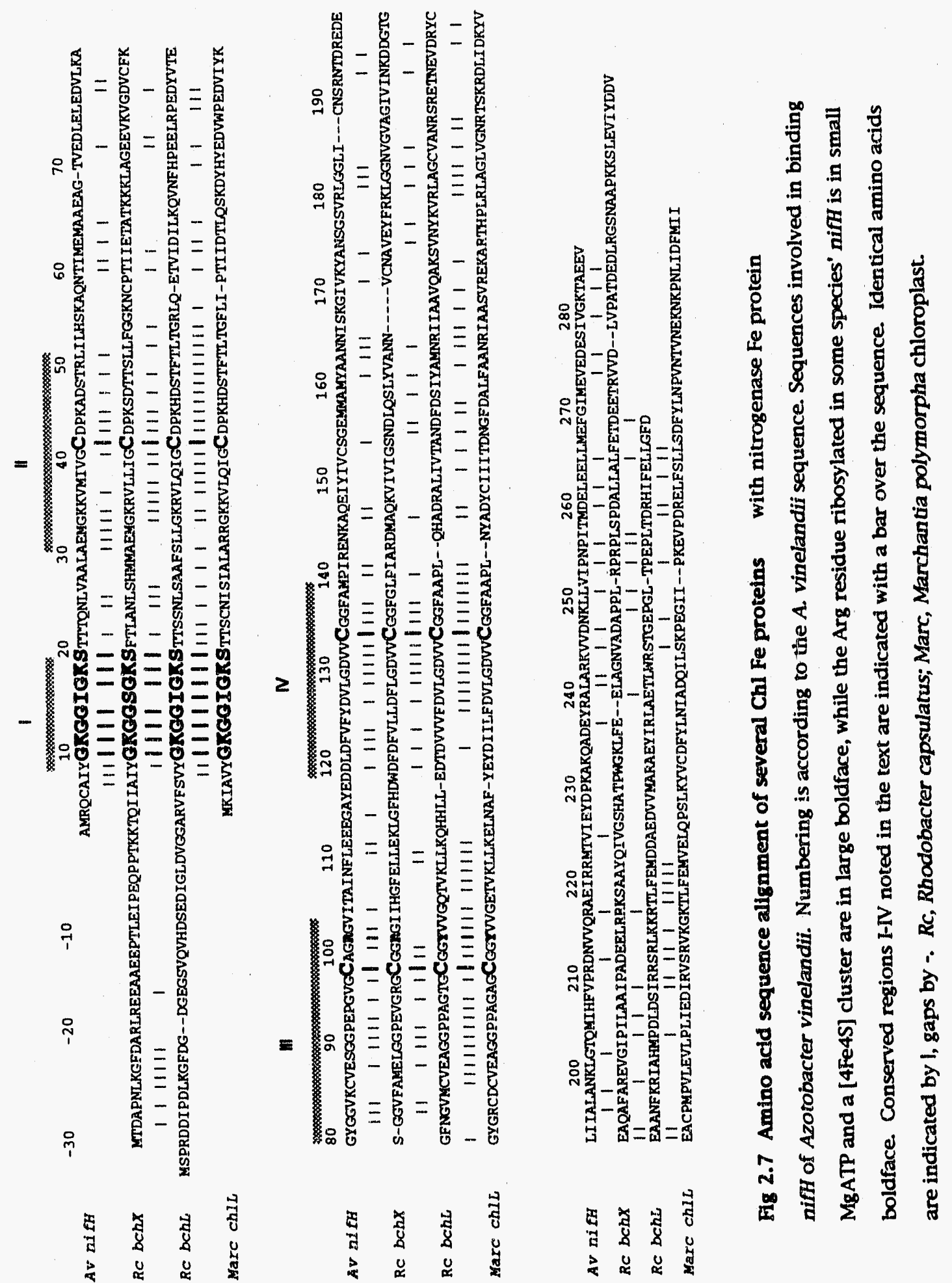


and Michel 1990) (fig 2.6). There are no conserved His residues in the alligned region which might serve as ligands to the central $\mathrm{Mg}^{++}$in the substrate. As expected, the two Rhodobacter sequences are more closely related (79\% over the triply aligned sequence) than is either with the Rhodopseudomonas sequence $(60 \%)$.

$b c h X$ shares $32 \%$ amino acid sequence identity with $b c h L$ from $R$. capsulatus and chlL (formerly $f r x C$ ) from cyanobacteria and many chloroplasts, as well as $30-37 \%$ with nifH (fig 2.7). Both bchL and chlL are required in their respective organisms for light-independent PChlide reduction (Fujita et al. 1992, Suzuki and Bauer 1992, Yang and Bauer 1990), which is chemically a similar reaction to chlorin reduction. ("frxC" is not a ferredoxin as its name would suggest. It is much larger than any known ferredoxin and lacks the unwavering pattern of clustered cysteines found in all known ferredoxins (Meyer 1988). Because of frxC's structural and functional similarities to the $\mathrm{BChl}$ biosynthesis enzyme $b c h L$, we suggest the more informative name chlL for this Chl synthesis gene.) The strongest similarities among the four genes $b \operatorname{ch} L, \operatorname{chlL}, b \operatorname{ch} X$ and $n i f H$, are in sequences known to bind MgATP and a [4Fe4S] cluster (fig 2.7).

\section{DISCUSSION}

The $b c h A$ region contain three genes.

We have shown that the bchA chlorin reductase locus of $R$. capsulatus contains three coding segments, $b c h X, b c h Y$, and $b c h Z$, which can all be expressed as separate polypeptides. This is in contrast to the previously held view of the $b c h A$ locus as containing a single gene which occupied most or all of the four kilobases between $b c h C$ and pufQ. Taylor et al. (1983) proposed that 
bchA was fully contained within the EcoRI-H fragment of pRPS404 (Taylor et al. 1983), because plasmid pRPSE30 bearing this DNA fragment complemented the point mutants bchA603 and bchAl65. Zsebo \& Hearst (1984), however, mapped a bchA transposon insertion mutant (KZR8G2) to the downstream EcoRI-Q fragment (Zsebo and E. 1984a). Based on the Taylor et al. conclusion that $b c h A$ was fully contained within EcoH, Zsebo and Hearst concluded that the mutation in EcoQ was polar on a "downstream" bchA gene. Later studies by Young et al. (1989) confirmed that sequences within EcoRI-Q are required for chlorin reductase activity. They also confirmed the results of Biel and Marrs (Biel and Marrs 1983) that the direction of transcription proceeds from crtEF toward the puf operon, as described here.

In contrast to the results of Taylor et al., however, Young et al. were unable to use pRPSE30 to complement the point mutations bchA603, bchA617, and $b \operatorname{chA} 305$, which appear to lie within either $b \operatorname{chX}$ or $b \operatorname{ch} Y$ (see fig 1a of Young et al.). A plasmid containing both adjacent EcoRI fragments $\mathrm{H}$ and $\mathbf{Q}$ (pDAY26), on the other hand, complemented all bchA mutants tested (Yuing et al. 1989). These results suggested one of two possibilities: i) that the bchA locus comprised a single large gene spanning from bchC to pufQ, as those authors concluded, or ii) that the mutations used in the complementation studies were polar with respect to the portion of $b c h A$ in $E \operatorname{coR} I-\mathrm{Q}$. Our data support the latter explanation, since there are clearly three genes between $b c h C$ and pufQ. There is also a preliminary report that a cosmid from Rhodospirillum centenum is able to complement some $R$. capsulatus bchA point mutants but not others (Bauer 1991d), providing further evidence for the plurality of chlorin reductase genes. 


\section{Translational initiation of bchZ and bchY.}

We have presented evidence that $b c h Z$ expression is coupled to $b \operatorname{ch} Y$ expression. Specifically, efficient translation of $b \operatorname{chZ}$ in $E$. coli is absolutely dependent on prior translation of $b c h Y$. Failure to initiate $b c h Y$ translation (as in $\mathrm{pDBX102c}$ ) results in a complete loss of detectable bchZ product.

Furthermore, when bchY translation is terminated prematurely (pDBX125) or

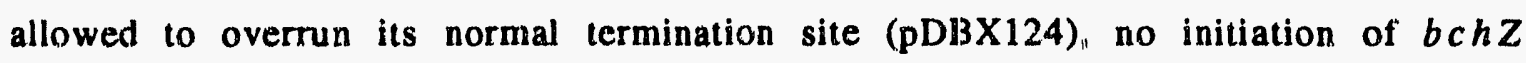
is detected. We conclude that a ribosome must be delivered to the bchZ start in cis by termination of bchY at its normal $3^{\prime}$ end in order for translation initiation to occur. Similar dependence on the location of the stop for an upstream gene of a translationally coupled pair has been observed for the trpB-trpA juncture (Das and Yanofsky 1989). The bchY lerminus is internal to the potential mRNA structure $(-9.9 \mathrm{kcal} / \mathrm{mol})$ at the $\xi^{\prime}$ end of $b c h z$. mRNA secondary structure in the neighborhood of translational start sequences has been shown on several occasions to influence the efficisncy of translation initiation (Altuvia et al. 1989, de Smit and van Duin 1990, Hartz, McPheeters, and Gold 1991). The stop/start overiap within the stem-loop is a classical arrangement in translational coupling, and one believed to permit the translating ribosome to iron out the structure occluding the downstream gene's initiation signals (Gussin 1966, Min Jou et al. 1972).

The stop for $b \operatorname{ch} X$, on the other hand, is 45 nucleotides prior to the AUG start of $b c h Y$, and the structure that occludes this start is very stable $(-31.7$ $\mathrm{kcal} / \mathrm{mol}$ ). While this structure is likely to form, it is unllikely that the ribosome could then tron it out once it had folded, having already terminated 16 nucleotides prior to reaching the structure. Indeed, in our $E$. coli expression systems, bchY translation is never initiated from its own RBS, but 
only from derived constructs lacking the ability to form such a large stable structure (pDBX123, 124, and 125). Formation of the secondary structure and blockage of $b \operatorname{ch} Y$ initiation is probably responsible for the loss of $b \operatorname{chZ}$ product in $\mathrm{pDBX} 102 \mathrm{c}$ and $\mathrm{pDBX} 204$. When the sequences containing this secondary structure were removed in an in-frame fusion between $b \operatorname{chX}$ and $b c h Y$ (pDBX105), protein synthesis continued to the end of bchY, and bchZ expression was restored. Differences in the velocities of $\mathrm{T} 7$ and $E$. coli RNA polymerases do not account for the excess of bchX expression over bchY and $b c h Z$, as pDBX204 does no better at expressing the downstream genes than does pDBX102c. $R$. capsulatus may have its own mechanism which allows translational initiation of bchY. A spontaneous or factor-assisted frameshift in the last 11 nucleotides of bchX, for example, would allow the ribosome to bypass the stop in the $b \operatorname{ch} X$ sequence, while placing it in the same reading frame as the UGA stop that overlaps the AUG start of bchY. Such a frameshift would facilitate translational initiation of $b \operatorname{ch} Y$ both by opening the secondary structure occluding its ribosome binding site and halting the ribosome immediately over the bchY start signals.

The significance of the translational coupling we observe in the $E$. coli T7 expression systern remains to be demonstrated in Rhodobacter. It also remains to be shown directly that all three genes are required for chlorin reductase activity. The existence of numerous mutations in EcoRI-Q giving rise to the BchA phenotype (Young et al. 1989, Zsebo and E. 1984a) makes a role for $b c h Z$ unequivocal. Likewise, the sequence similarities between $b c h X$ and bchL provide both a mechanistic and an evolutionary argument for the involvement of bchX in chlorophyllide reduction (see nexi section). However, the only evidence so far for the involvement of bchY in this enzymatic step is 
its homology with a subunit of the light-independent PChlide reductase, $b c h N$ (chlN in plant chloroplasts) (Burke, Alberti, and Hearst 1992b). Since. disruption of bchY translation results in loss of bchZ expression, any mutation in chlorin reductase which maps to $b \operatorname{ch} Y$ must be shown not to be polar on $b c h Z$ before the role of bchY will be resolved.

\section{Chlorophyll Fe proteins.}

The unexpected link between two photosynthetic pigment synthesis reactions and nitrogen fixation suggests that all three processes are related mechanistically, structurally, and evolutionarily. They also define a new class of enzymes, the Chl Fe proteins, with properties that are similar to those of nifH. Details of the evolutionary implications of these alignments are presented in Chapter 4 , while the extent to which nifH can be used as a model for both the mechanism and some of the details of the tertiory structure of the Chl Fe proteins is outlined below.

Two cysteines from each subunit of a nifH homodimer bind a [4Fe4S] cluster (Howard et al. 1989a, Orme-Johnson 1985). These Cys residues and their sequence contexts are shared between the nifH proteins and the $\mathrm{Chl} F$ proteins (sites III and IV in fig 2.7), so $b c h X, b c h L$, and chlL are each strongly predicted to bind [4Fe4S] clusters as homodimers, hence their designation as Chl Fe proteins. In addition, all the proteins in fig 2.7 share the sequence $(\mathrm{A} / \mathrm{S})(\mathrm{I} / \mathrm{V}) \mathrm{GKGG(I/S)} \mathrm{GKS}(\mathrm{F} / \mathrm{T}) \mathrm{T}$ (site 1$)$. The sequence $\mathrm{G} \times \mathrm{G} \times \mathrm{GKK}(\mathrm{T} / \mathrm{S})$ is conserved in a large number of ATP binding proteins (Albright et al. 1989, Higgins et al. 1986). A third cysteine shared among all the proteins in fig 2.7 (site II) has been shown to be important for efficient nitrogenase function (Howard et al. 1989a), and may be involved in ATP-binding or transduction of 
the signal of ATP hydrolysis to the [4Fe4S] cluster (Hausinger and Howard 1983, Howard et al. 1985). The Chl Fe proteins, then, are predicted to bind ATP or MgATP in a manner that is structurally similar to the binding of MgATP by nitrogenase Fe protein and to utilize ATP hydrolysis as part of their enzymatic mechanism.

Based on these observed similarities, we propose a general model for the mechanism of reduction by complexes utilizing nifH-like Fe:S proteins (fig 2.8), in which the $\mathrm{Fe}: S$ protein (nifH in nitrogen reduction, $b c h X$ in chlorin reduction, bchL in PChlide reduction in Rhodobacter, or chlL in PChlide reduction in cyanobacteria and chloroplasts) serves as unique electron donor to the catalytic portion of the complex (nifDK, bchYZ, bchNB, or chlNB (Burke, Alberti, and Hearst $1992 \mathrm{~b})$ ). In $\mathrm{N}_{2}$ reduction, MgATP binding to reduced nifH protein exposes the Fe:S cluster and enhances the reduction potential from $0.29 \mathrm{~V}$ to $-0.40 \mathrm{~V}$. Hydrolysis then accompanies electron transfer to, and dissociation of nifH from, the MoFe protein (nifDK), where $\mathrm{N}_{2}$ reduction takes place (Orme-Johnson 1985). It is very likely that ATP hydrolysis and transfer of electrons through a $[4 \mathrm{Fe} 4 \mathrm{~S}]$ cluster also play pivotal roles in the dark reductive reactions of $\mathrm{Chl}$ and $\mathrm{BChl}$ syntheses. ATP-induced allostery may also occur, perhaps involving the residues at site II (see above). The Chl Fe proteins then either pass electrons to the other two subunits of the complex where substrate reduction takes place, or they may reduce their substrates directly. Several aspects of this mechanism have been confirmed experimentally for the chlL gene product, which bound Cibacron Blue and Procion Red affinity columns (indicating probable ATP binding) and eluted as a dimer from gel filtration columns (Fujita et al. 1989), but there is not yet any direct experimental evidence for the $b c h L$ or $b c h X$ gene products. This 

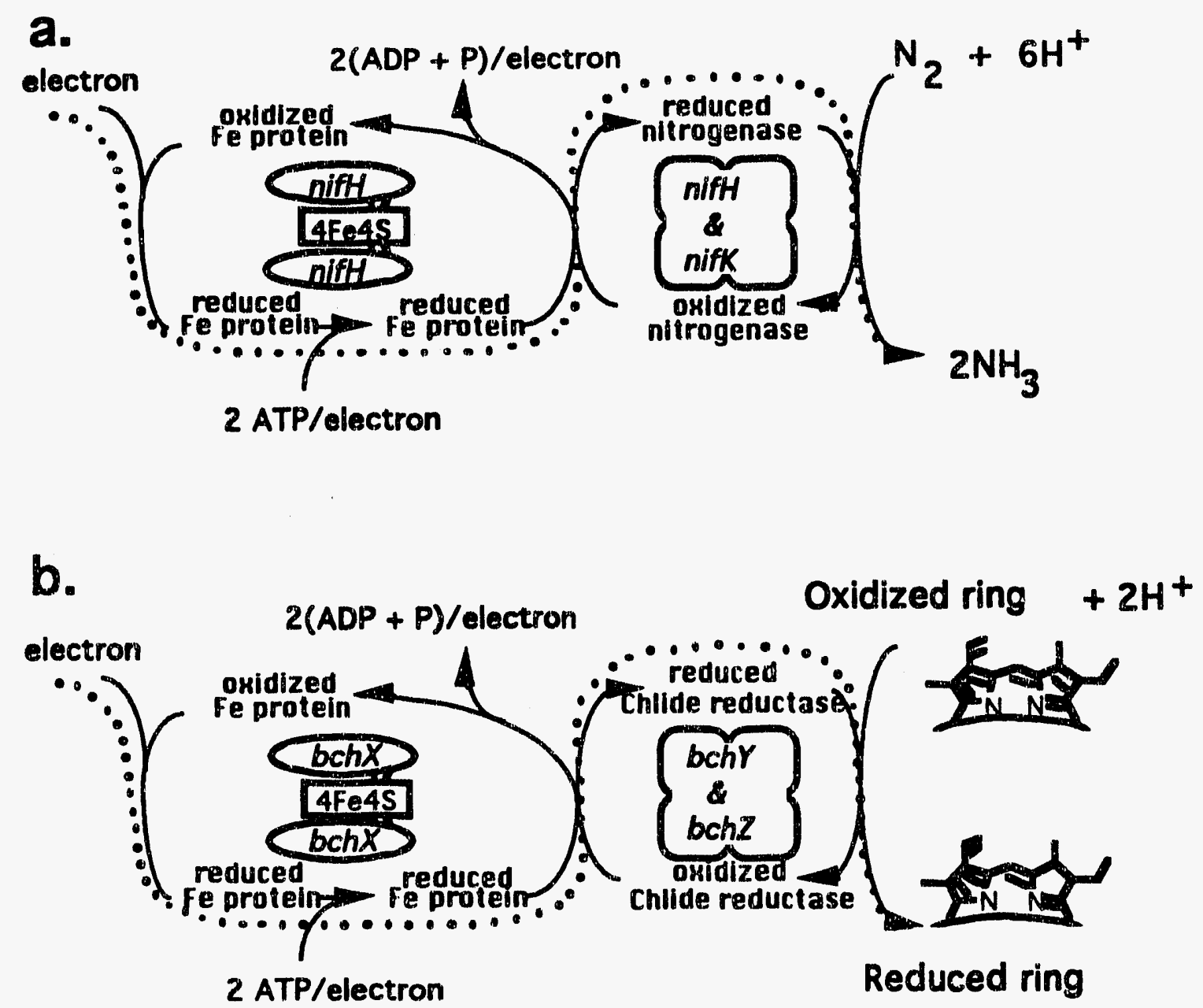

Fig 2.8. Proposed mechanism for light-independent PChlide and Chlide reductions as described in the text, using as example the chlorin reductase encoded by bchXYZ. (a) Mechanism of nitrogenase and nitrogenase reductase. (b) Chlorin reductase. Dots show the pathway of the reducing electrons from an anonymous donor to the tetrapyrrole macrocycle. An analogous diagram could be drawn for PChlide reduction by $b c h L N B$ in purple bacteria or chlLNB in chlorophyllous organisms. 
mechanism for dark PChlide reduction is in contrast to that of the lightdependent reaction, in which the reductase is encoded by a single gene (Schultz et al. 1989), and absorbance of a photon by PChlide is required for its reduction by NADPH (Griffiths 1991).

An alternative model would have the [4Fe4S] cluster participate directly in the redox catalysis in a manner analogous to nitrite reductase and the $\beta$ subunit of sulfite reductase (Ostrowski et al. 1989). In each of these cases, an electron is transfered directly from a [4Fe4S] cluster to siroheme, which is liganded by one of the sulfurs in the cluster. It is not known whether the electron is transfered directly to the Fe atom through a bridging cysteinyl sulfur or through the $\pi$-ring system of the tetrapyrrole (Ostrowski et al. 1989). Similar prosthetic group-substrate interactions could potentially occur with the $\mathrm{Chl}$ Fe proteins.

The nitrogenase Fe protein appears to be an excellent model not only for the mode of action of Chl Fe proteins, but for some of the details of their three-dimensional structures as well. First, when conserved regions I-IV are mapped onto the Azotobacter vinelandii nifH crystal structure (Rees 1991), a number of the matches are located both within the subunit interiors and at the subunit-subunit interface of the homodimer. Some of these residues are involved in salt bridge and van der Waals contacts across the subunit-subunit interface, suggesting similar interhomodimeric interactions. Second, Arg100, which is reversibly ADP-ribosylated in the nifH of some species, is conserved in $b c h X$. This does not necessarily imply that ADP-ribosylation plays a part in the regulatory cycle of the $b \operatorname{ch} X$ gene product, since $\operatorname{Arg}^{100}$ is universally conserved among nifH protein, while ADP-ribosylation is limited to a few 
species. It may instead reflect similar structural and functional constraints operating on both the nitrogenase and $\mathrm{Chl} F$ pe proteins. $\operatorname{Arg} 100$ is replaced by Tyr in all of the PChlide reductases. Wolle et al. (1992) have shown that $R^{100}$.$>\mathrm{Y}^{100}$ is the most readily tolerated substitution in the $A$. vinelandii nitrogenase Fe protein at this position. Third, there are a large number of acidic residues between sites III and IV in all of the proteins in fig 2.7. This region has been shown to be involved in ionic interactions of nifH with nifD ( $\beta$-subunil of MoFe protein) (Deits and Howard 1990, Willing and Howard 1990, Willing et al. 1989, Wolle et al. 1992), suggesting that ionic interactions may bind the $\mathrm{Chl} F e$ proteins to their ancillary proteins, namely $b c h Y Z, b c h N B$, or chlNB. Finally, using the structural compatibility method of Bowie $e t$ al., which assesses the likelihood of packing and exposing residues in a given sequence (Bowie, Luithy, and Eisenberg 1991), the $\mathrm{Chl} F \mathrm{Fe}$ proteins are all predicted to be compatible with adopting a nifH-like structure (Eisenberg 1991b). In contrast to the similarities noted above, bchX and bchL are unique in possessing similar $\mathrm{N}$-terminal extensions containing a large number of charged residues (13/36 in each).

Nitrogen fixation and purple bacterial (i.e., Proteobacterial) photosynthesis are both anaerobic processes. The half-life of the nifH [4Fe4S] cluster in air is approximately 30 seconds (Mortenson and Thornley 1979). $b c h X, b c h L$, and $c h l L$ may also be inactivated by aerobic conditions. Indeed, BChl synthesis is immediately suppressed in Rhodobacter by the introduction of oxygen (Cohen-Bazire, Sistrom, and Stanier 1957, Lascelles 1975). In addition to transcriptional regulation by oxygen levels (Biel and Marrs 1983, Ma 1992a, Sganga and Bauer 1992, Zhu et al. 1986a, Zhu and Hearst 1986b), anaerobic regulation of $\mathrm{BChl}$ biosynthesis may also be directly mediated by 
oxygen-sensitivity of the enzymes themselves. This presents a paradox for green, oxygenic organisms, which must reduce PChlide to produce chlorophyll in order to do oxygenic photosynthesis. Respiratory protection of nitrogenase by a vigorous, oxygen-consuming metabolism has been demonstrated in obligately aerobic, nonphotosynthetic bacteria such as Azotobacter (Mortenson and Thornley 1979, Yates and Jones 1974) and for heterocystic (Wastyn et al. 1988, Winkenbach and Wolk 1973) and unicellular (Peschek, Villgrater, and Wastyn 1991) nitrogen-fixing cyanobacteria. If on the other hand, the cellular interiors of actively oxygen-evolving photosynthesizers is not generally anaerobic, their chlL may well have diverged sufficiently from nifH to make them resistant to significant oxygen concentrations. If they have, it may eventually be possible to redesign nitrogen fixing enzymes to make them oxygen tolerant as well. 
blank page for pagination only 
Chapter 3: The $b c h F N B H$ bacteriochlorophyll synthesis genes of Rhodobacter capsulatus, and identification of the third subunit of light-independent protochlorophyllide reductase in bacteria and plants.

\begin{abstract}
In this chapter, I present the nucleotide and deduced amino acid sequences of four BChl-synthesis genes from the $7.1 \mathrm{~kb}$ BamD fragment of $R$. capsulatus. The pigments accumulated in strains with physically mapped mutations are analyzed by absorbance, fluorescence emission and fluorescence excitation spectroscopy, allowing us to assign the four open reading frames in BamD as $b c h F, b c h N, b c h B$, and $b c h H$. bchF encodes a bacteriochlorophyll-specific enzyme that adds water across the 2-vinyl group. The other three genes are required for portions of the pathway that are shared with chlorophyll synthesis, and expected to be common to both pathways. $b c h N$ and $b c h B$ are both required for dark protochlorophyllide reduction (along with $b c h L$ ), a reaction that has been observed in all major groups of photosynthesizers except angiosperms, where the reaction is lightdependent. The similarity of the purple bacterial enzymes to the plant enzymes is underscored by the fact that $b \operatorname{chN}$ is $35 \%$ identical to $\operatorname{chlN}$ (gidA), and $b c h L$ is $49 \%$ identical with chlL (gidB, frxC). Furthermore, bchB is $33 \%$ identical to ORF513 from the Marchantia polymorpha chloroplast. We present arguments in favor of the probable role of ORF513 (chlB) in dark protochlorophyllide reduction, as well as the similarity between protochlorophyllide reduction in both $\mathrm{Chl}$ and $\mathrm{BChl}$ synthesis and the subsequent chlorophyllide reduction in BChl synthesis. Finally, the probable role of $b c h H$ in methylation of the ring $\mathrm{C}$ propionic acid is discussed.
\end{abstract}




\section{INTRODUCTION}

Dark synthesis of chlorophyll (Chl) has been recognized for a long time (Bogdanovi'c 1973), and yet it has received little attention until recently. In part this has been because of the lack of genetic and biochemical starting points for studying the reaction, and in part because of the too often repeated refrain that plants do not make chlorophyll in the dark. Indeed, protochlorophyllide (PChlide) reduction in angiosperms is strictly lightdependent. Both PChlide and its light-dependent reductase accumulate in etiolated angiosperm scedlings, making both the enzyme and the reaction relatively easy to study (reviewed in ref (Griffiths 1991)). However, a diverse array of photosynthetic organisms including pine, algae, cyanobacteria, and purple bacteria are capable of dark $\mathrm{Chl}$ synthesis. The purple bacterium Rhodobacter capsulatus induces bacteriochlorophyll (BChl) synthesis and a host of other processes upon a drop in oxygen levels in a metabolic switch from aerobic respiratory to anaerobic photoheterotrophic growth (CohenBazire, Sistrom, and Stanier 1957). BChl and Chl synthesis, which are reviewed by Leeper (Leeper 1991), proceed by similar pathways until the production of chlorophyllide (Chlide). Addition of a phytol tail to Chlide produces Chl, while reduction of the chlorin ring and additional Bchla-specific reactions occur before addition of the phytol tail in BChl synthesis (fig 1.8). $R$. capsulatus accumulates $\mathrm{BChl}$ anaerobically in the dark provided that there is a suitable alternative electron acceptor in the medium, such as DMSO (Madigan and Guest 1978, Schultz and Weaver 1982, Yen and Marrs 1977). Its BChi-synthesizing enzymes, including both the PChlide and chlorin reductases, must therefore function independently of light. 
The first mutants in $R$. capsulatus that were linked to a block in PChlide reduction were designated $b c h B$ (Yen and Marrs 1976), although the location of this gene was never determined precisely. Coomber et al. and Yang and Bauer later showed that bchL (Zsebo and E. 1984a) is also required for PChlide reduction (Coomber et al. 1990, Yang and Bauer 1990), leading to independent speculation from both groups that PChlide reductase might be a multisubunit enzyme in $R$. capsulatus. The amino acid sequence of the bchL gene product is highly homologous (48-50\%) with the chlL gene (also called $f r x C$ and gidB, for Greening In the Dark) from cyanobacteria (Burke, Alberti, and Hearst 1991, Fujita et al. 1991), from the cyanelle of the flagellar eukaryote Cyanophora paradoxa (V. L. Stirewalt and D. Bryant, unpublished), and from the chloroplasts of the alga Chlamydomonas reinhardtii (Suzuki and Bauer 1992), the nonvascular plant Marchantia polymorpha (Ohyama et al. 1986), the fern Polystichum acrostichoides (Burke, Alberti, and Hearst 1991) and many conifers (Burke et al. 1992d, Lindholm and Gustafsson 1991). The ChlL gene product is localized to the chloroplast (Fujita et al. 1989), and like its purple bacterial counterpart bchL, chlL is required for dark PChlide reduction (Fujita et al. 1991, Suzuki and Bauer 1992). Another chloroplast gene, chlN (also known as gidA), has also been shown to be required for dark PChlide reduction (Choquet et al. 1992, Roitgrund and Mets 1990), and chlN sequences have been obtained from the chloroplasts of $C$. reinhardtii (Choquet et al. 1992), Marchantia polymorpha (ORF465 (Choquet et al. 1992, Shinozaki et al. 1986)), and Pinus negra (Lindholm and Gustafsson 1991). Neither chlL nor chlN sequences are present in the sequenced chloroplast genomes of rice (Hiratsuka et al. 1989) or tobacco (Shinozaki et al.1986). There are also a number of nuclear mutations, the 6o-called yellow mutations, in Chlamydomonas reinhardtii that cause a block in dark PChlide reduction (Ford, 
Mitchell, and Wang 1981, Ford and Wang 1980a, Ford and Wang 1980b, Wang 1979), although the nature of the yellow mutations has not yet been deduced. In short, two sequenced chloroplast genes, chll and $c h l N$, are known to be required for light-independent PChlide reducti $n$ in Chl-containing organisms, and two genes are known for Rhodobacter, bchB and bchL, of which only the sequence of the latter has been published (Yang and Bauer 1990, Youvan et al. 1984a).

Recently it has been shown that chlorin ring reduction is catalyzed by a multisubunit enzyme complex encoded by the $b c h X Y Z$ locus (formerly $b c h A$, see fig 1.8) (Burke, Alberti, and Hearst 1992a). Chemically, the reduction of ring $B$ of the macrocycle by chlorin reductase is nearly identical to PChlide reduction, in which ring $D$ is reduced. Therefore it is of great significance that $b c h X$ is homologous with $b c h L(32 \%)$, and with the nitrogenase Fe protein nifH (32\%) (Burke, Alberti, and Hearst 1992a, Hearst, Alberti, and Doolittle 1985). Because of their similarities with nifH, the Fe:S-containing pigment synthesis enzymes, $c h l L, b c h L$, and $b c h X$ are known collectively as the Chl Fe protein family (Burke, Alberti, and Hearst 1992a). We have proposed a mechanism for dark PChlide and Chlide reduction based on the conservation of key amino acid sequences between the $\mathrm{Chl}$ Fe proteins and nifH, in which each Chl Fe protein serves as a unique electron donor for the catalytic portion of its respective complex (Burke, Alberti, and Hearst 1992a). As an extension of this model, it would be logical to postulate that the catalytic, substrate binding portions of the two complexes would also be similar. An asymmetry between Chlide and PChlide reductions is immediately apparent, however, in that while two ancillary proteins (bchY and $b c h Z$ ) are known for the reaction involving 
$b c h X$, only one has been suggested to work in concert with $b \operatorname{chL}(b c h B)$ or with $\operatorname{chlL}(\operatorname{chlN})$.

The precise location of the $b \operatorname{ch} B$ gene of $R$. capsulatus has not been determined, but it is commonly believed to fall within the BamHI-D (BamD) fragment of pRPS404, an R-prime plasmid (Marrs 1981) which carries $46 \mathrm{~kb}$ of mobilized $R$. capsulatus chromosome encoding nearly all of the functions required for photosynthesis (see, for example fig 1 of (Yang and Bauer 1990)). Three other genes have also been mapped to BamD: bchF mutants fail to add water across the vinyl group on ring $A$ in a Bchla-specific reaction, and thus accumulate 2-vinyl-bacteriochlorophyllide $a, b c h H$ mutants accumulate protoporphyrin IX. They are presumed to be deficient in both the methyltransferase and the $\mathrm{Mg}$ chelatase, since these two steps appear to be obligately coupled in Rhodobacter (Biel and Marrs 1983, Gorchein 1972, Gorchein 1973). Finally, bch $K$ has been thought to work at the same step as bchH (Zsebo and E. 1984a), although as we show here, it acts much later in the pathway, and is identical to $b \operatorname{ch} B$.

We have sequenced BamD and analyzed mutations mapping within this fragment. Our results allow us to assign four BChl synthesis genes, including two for PChlide reduction, $b c h B$ and $b c h N$. Furthermore, by comparing our sequences with those in protein sequence databases, we are able to assign tentatively the third subunit of light-independent PChlide reductase (chlB) to Marchantia chloroplast ORF513. 


\section{Materials and Methods}

General. Plasmid pFL120 contains the $7.1 \mathrm{~kb}$ BamHI-D fragment of pRPS404 (BamD) (Marrs 1981) subcloned into the BamHI site of pBR325. 136 M13 clones spanning all of BamD in both directions 2- to 3-fold vrere subcloned from pFL120 for single strand, dideoxy chain-terminating sequencing (Burke, Alberti, and Hearst 1992a, Sanger, Nicklen, and Coullson 197\%) in both directions as described previously (Armstrong et al. 1989a). pFL120 and M13 subclones were maintained in E. coli strains $\mathrm{HB} 101$ and JM103, respectively. Ampicillin was used at a concentration of $100 \mu \mathrm{g} / \mathrm{mll}$.

DNA extractions and analysis. DNA was isolated from wild type $R$. capsulatus strain SB1003 and from Tn5.7 transposon insertion mutant strains KZR8G9, KZR9E11 (Zsebo and E. 1984a). The 2.85 BamHl-Sall fragment of pFL120 was isolated from a $0.8 \%$ agarose gel run in $1 \mathrm{x}$ TAE buffer (Sambrook, Fritsch, and Maniatis 1989), purified using the GeneClean kit (Bio 101, Inc., La Jolla), and radiolabeled by random hexamer priming using [ $\alpha_{\mathrm{m}} 32$ P]dATP (Sambrook, Fritsch, and Maniatis 1989) Southern transfer and hybridization were performed according to standard procedures (Sambrook, Fritsch, and Maniatis 1989), and the radioactive blots were analyzed by PhosphorImage scanning prior to exposure to film.

Pigment extractions. Freshiy saturated $R$. capsulatus cultures grown aerobically in RCV medium (Weaver, Wall, and Clest 1975a) were diluted 4- to 5fold in $\mathrm{RCV}^{+}$medium (Yen and Marrs 1977), which contains DMSO as terminal oxidart to allow dark anaerobic induction of bacceriochlorophyll synthesis. 25 $\mathrm{ml}$ of this culture was harvested after 2.5 days induction and the pellet was crisacted iwice with son 0 ! of acetonemembno! (7:2) or methanol a!one. 


\begin{abstract}
Absorbance spectra were taken on a Hewlett Packard 8452A diode array spectrophotometer. Extracts were diluted to an optical density nar 0.1 at the red-most absorption peak for use in fluorometric analysis in order to minimize self-absorbance. Fluorescence emission and excitation spectra were taken on a SPEX fluorolog $212 \mathrm{~T}$ fluorimeter. All spectral data are given for the acetone:methanol extracts, except where otherwise noted.
\end{abstract}

Computer analysis. Protein (NBRF-PIR 29 and SwissProt 19) and nucleic acid (six translated frames of Genbank 69 and EMBL 28) databases were searched with the fastdb program using Intelligenetics Software release 5.37 (Brutlag et al. 1990) with parameters set so as to maximize their normalized alignment scores (N.A.S.) (Doolittle 1986). Three-way alignments were refined by hand using the same N.A.S. criteria. Gapped positions were ignored in calculating percent identities. Hydrophobicity plots were calculated using the Kyte-Doolittle (Kyte and Doolittle 1982) hydrophobicity indices averaged over a window of 19 amino acids. The DNA sequences presented in this paper are on deposit in the EMBL. DNA sequence databank under accession number Z11165.

\title{
RESULTS
}

\section{Sequence of the four open reading frames of Bamb and} identification of potential promoters.

The nucleotide and deduced amino acid sequences of BamD are shown in figure 3.1. BamD is 7103 nucleotides in length and contains four open reading

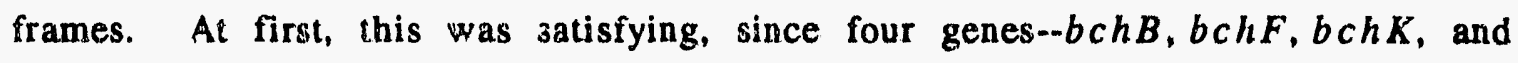
bchH--have been mapped to BamD (Biel and Marrs 1983, Taylor et al. 1983, Zsebo and E. 1984a). However, as we show below, all but one of these four are 


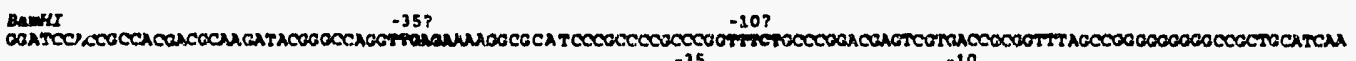

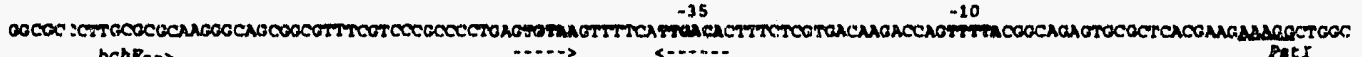

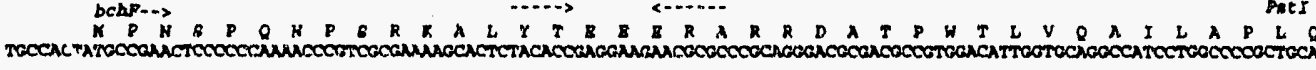

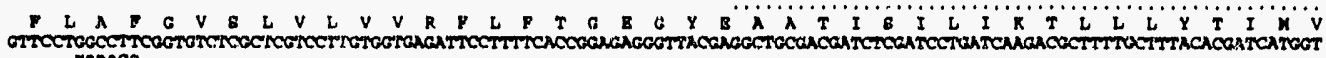

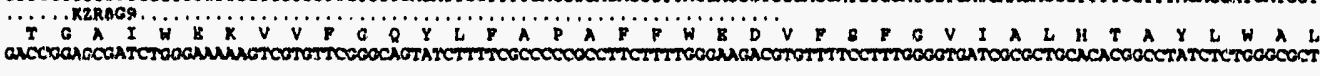

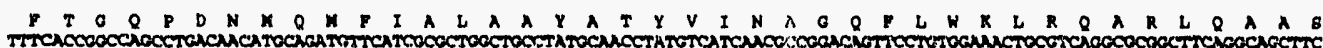

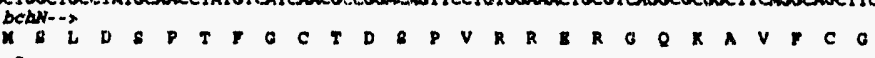

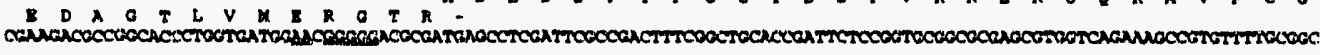

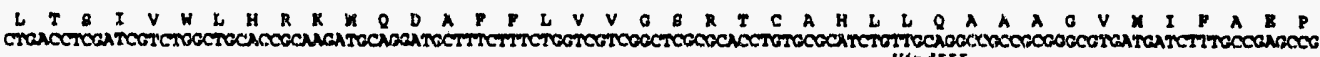

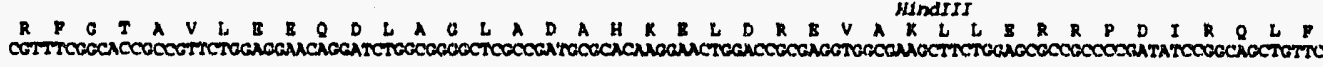

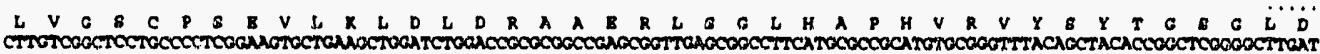

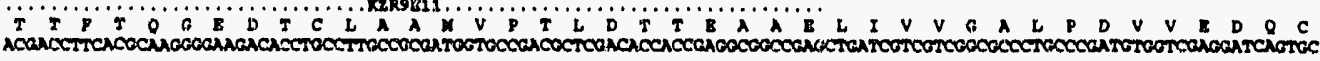

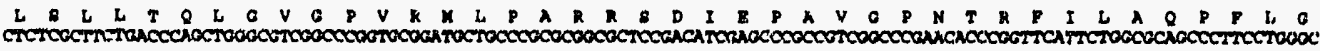

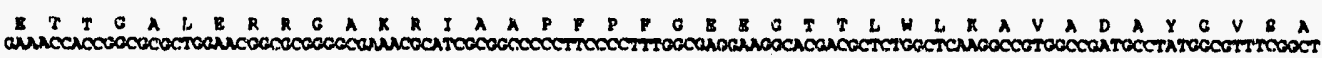

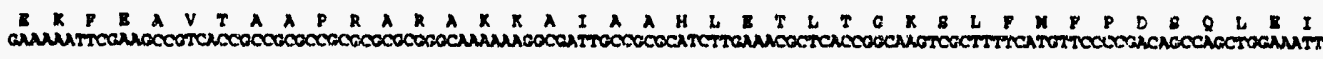

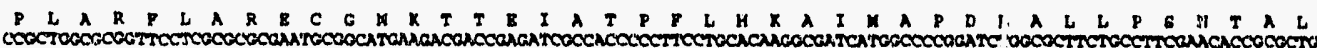

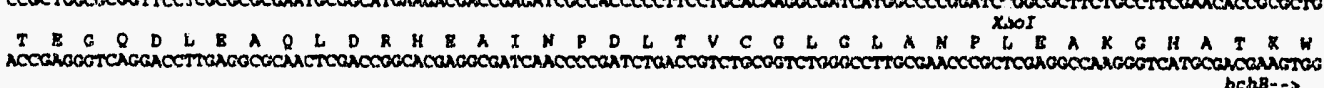

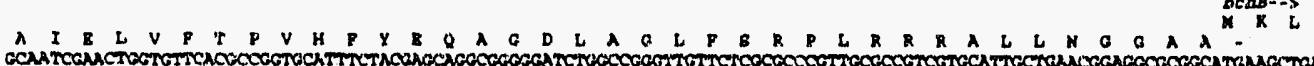

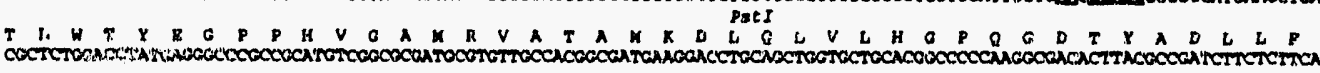

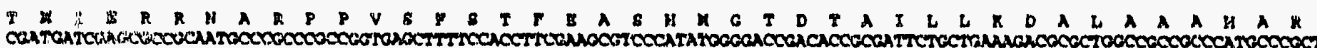

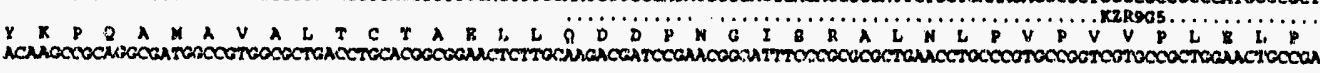

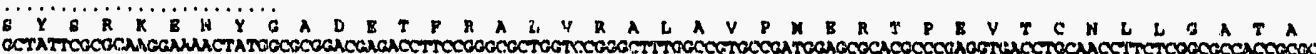

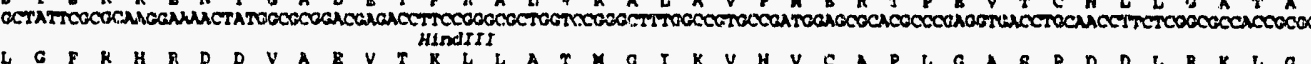

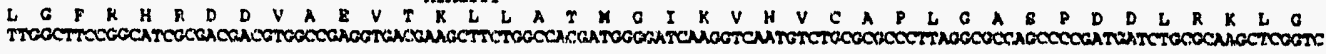

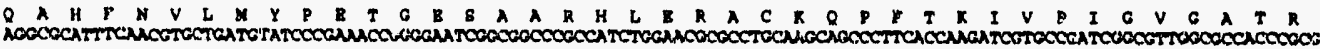

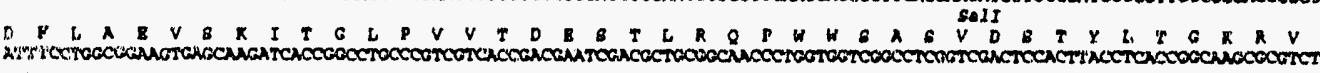

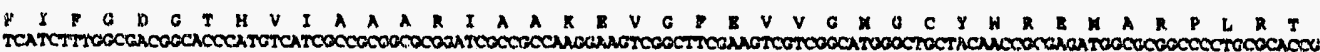

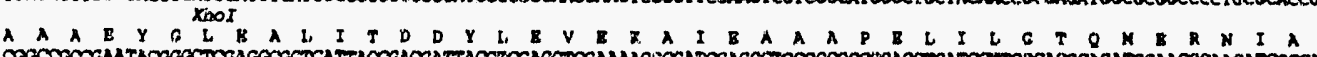

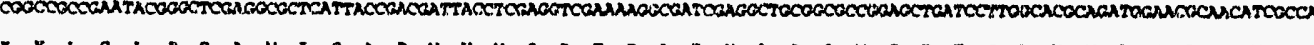

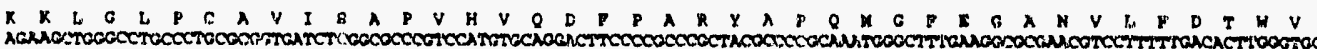

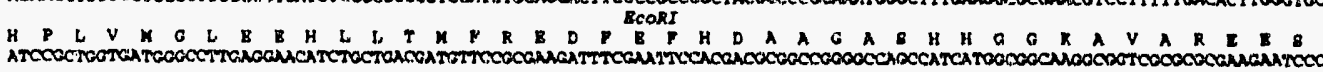

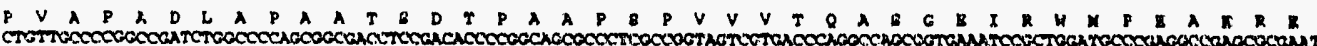

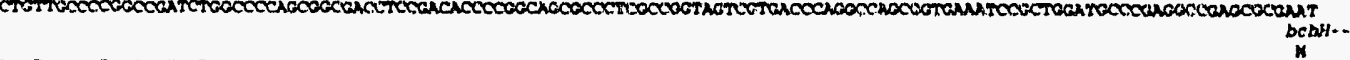

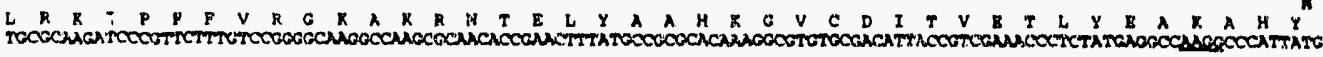

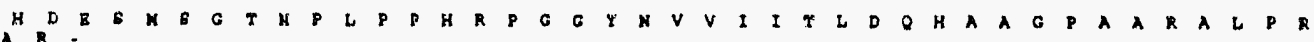



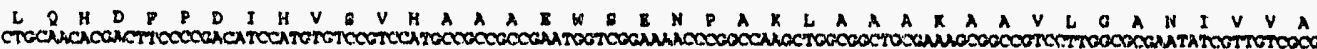

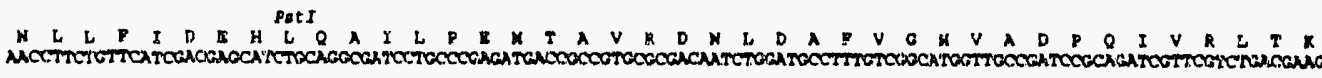

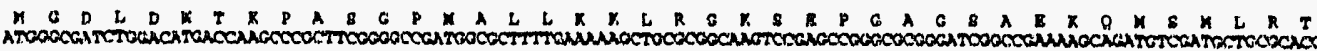

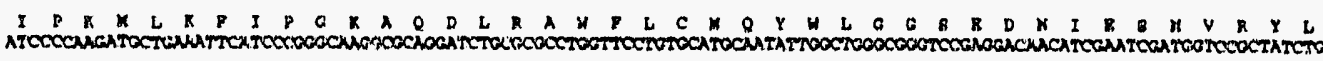

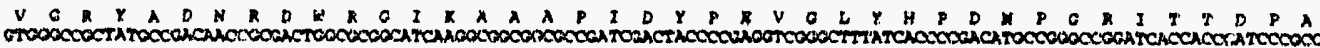

13712 13592 38
13472 1335 128
13233 158
131212 27
171 12992 67
23872 107
12752 147
12632 187
12512 227
12392 267
12272 307
12152 3077
12033 367
191212 3
424
11792 . 11672 21552 1233
12632 12532 203 263
21072 293
20952 2033 363
10712 10592 (1) 10672 20353 5533
10232
.123 51
525
10212 9992 9672 9281 963 201
95212 


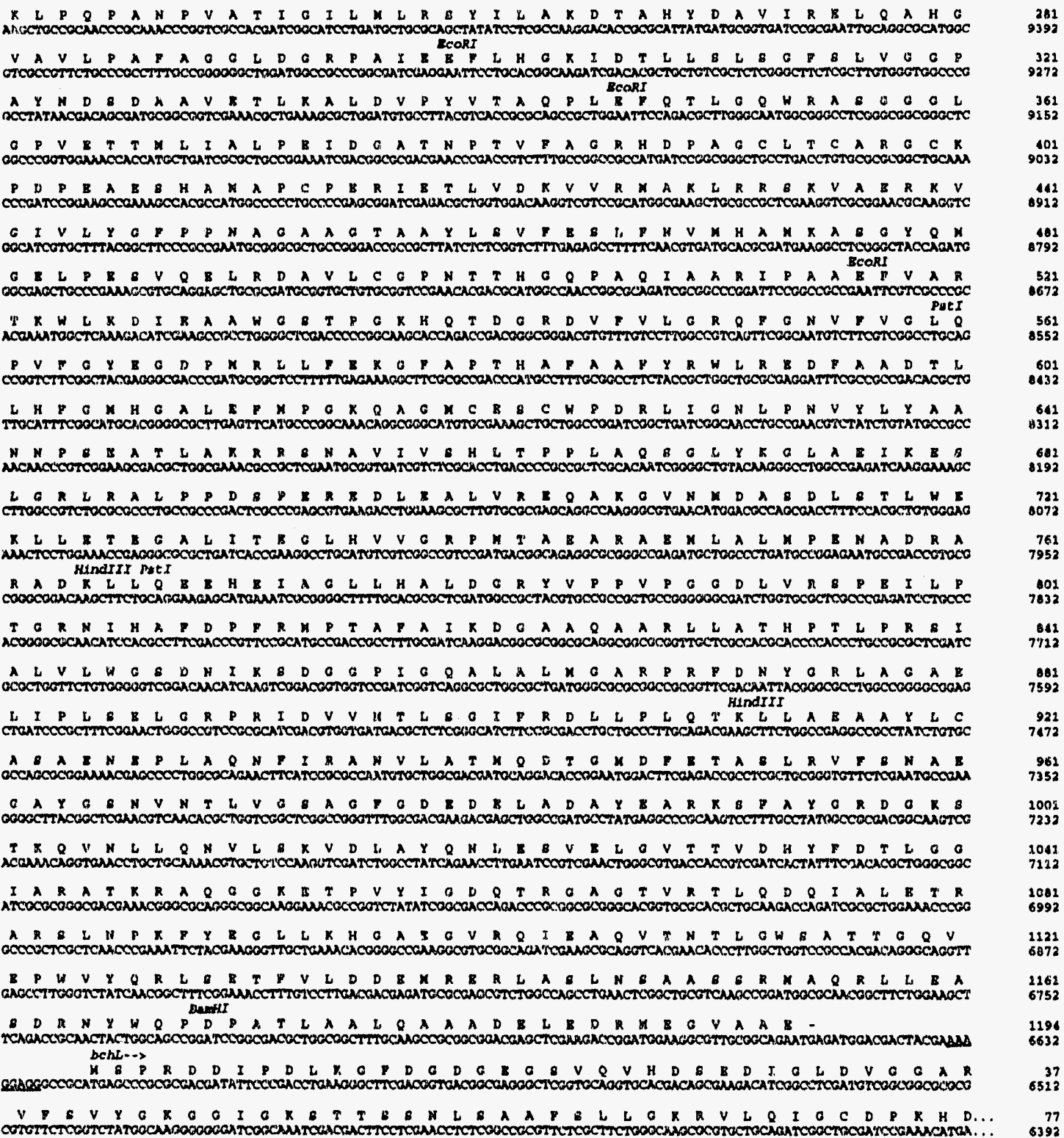

fig. 3.2 Nucleotide and deduced amino acid sequences of EamD. Potential $\mathbf{5} 70$ promoters are shown in bold and the pallindromic control sequence is shown with arrows. Ribosome binding sites are underlined. Cleavage sites for restriction enzymes used in this study are shown above the sequence, as are the mapped locations of three Tn5.7 transposons with approximate error bars given as dots (see Table 1). Amino acid and nucleic acid sequence numbering is given in the right-hand margin. Nucleic acid numbering corresponds to that of the photosynthesis gene cluster (EMBL DNA sequence databank accession \# Z11165). 
in a different crder and/or perform different functions than has been ascribed to them.

There is a potential $\sigma^{70}$ promoter (TTGACA(N 20$)$ ITITA) 20 b.p. from the AUG start which overlaps a palindrome sequence (TGTAA $\left.\left(N_{7}\right) \operatorname{TIGACA}\right)$ proposed to be required for normal regulation of pigment synthesis genes by oxygen (Armstrong et al. 1989a, Ma 1992a). (Matches with consensus $\sigma^{\text {?n }}$ and palindrome sequences are underlined.) The coding sequence of each sene overlaps 1-8 n.t. with that of the next gene downstream, implying that a single polycistronic mRNA molecule may express all of the genes of BamD. The fourth ORF extends 73 n.t. into the next Bam fragment downstream (BamF), and so the four ORFs of BamD appear to form a continuous operon with $b \operatorname{chL}, b \operatorname{ch} M$, and ORF 1695, as has been proposed previously (Bauer et al. 1991a, Yang and Bauer 1990).

\section{Mapping the locations of transposon mutations.}

The fourth ORF has been shown to encode bchH (Biel and Marrs 1983, Coomber et al. 1990, Yang and Bauer 1990, Zsebo and E. 1984a). Three transposon Tn5.7 insertion mutations lie within BamD upstream from $b c h H$ (Zsebo 1984c, Zsebo and E. 1984a). The location of each was confirmed and refined by digesting chromosomal DNA from each strain with several different restriction enzymes. Wild-type strain SB1003 served as control. A Southern blot of digested DNA was then hybridized with a radioactive probe containing the first $2.85 \mathrm{~kb}$ Bam-Sal fragment of BamD, encompassing all of the first two and most of the third open reading frames. BamHI does not cut in Tn5.7, and so the $7.1 \mathrm{~kb}$ wild type chromosomal BamHI fragment is shifted to significantly greater than $12 \mathrm{~kb}$ in each of the mutant strains, due to the 


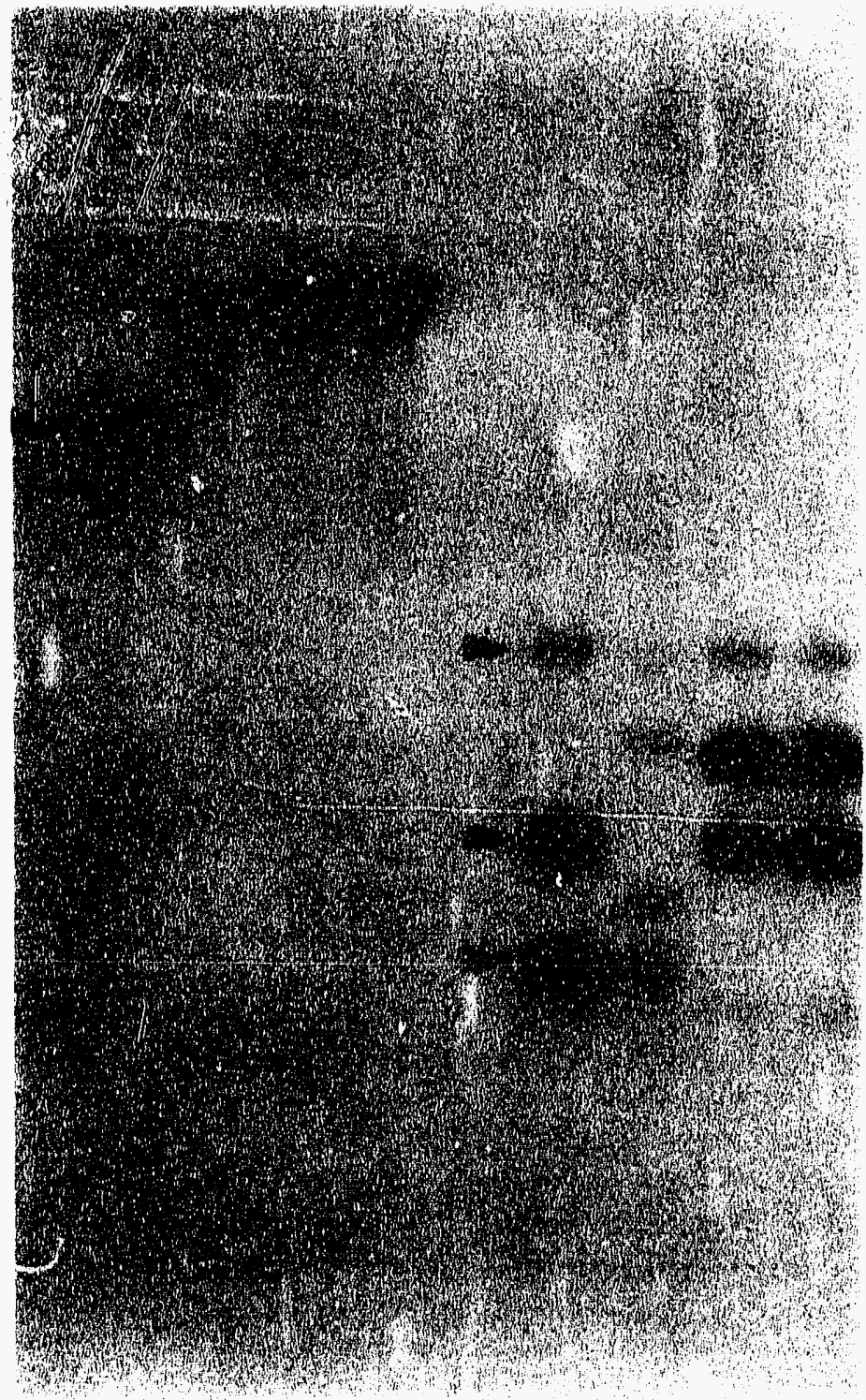

XBB $923-1629 \mathrm{~A}$ 
Fig 3.2. Mapping the locations of Tn5.7 insertions. Southern hybridizations to digested $R$. capsulatus DNA. Lanes 1, 5, wild type SB1003; lanes 2, 6, KZR8G9; lanes 3, 7, KZR9E11; lanes 4, 8, KZR9G5. DNA was digested with BamHI (lanes 1-4) or HindIII (lanes 5-8). The probe used was the 2.85 BamHI-SalI fragment of pFL120 labeled with 32p as described in Methods. 
presence of the transposon Tn5.7 (fig 3.2, lanes 1-4). There is only one band present in each of these lanes, indicating that the wild-type copy of this region is not present in the cell, as would have occured through a single recombination event. HindIII, BglI, PstI, and XhoI all cleave in the transposon. The wild-type restriction fragment containing the transposon is split into two fragments, each containing transposon DNA in addition to $R$. capsulatus DNA. Subtracting the contributions from the transposon DNA gives the distance to the insertion site. The results of mapping experiments using these enzymes uniquely place one transposon within each ORF (see fig 3.1).

Phenotypes of Transposon insertion mutants.

During the synthesis of both $\mathrm{Chl}$ and $\mathrm{BChl}$, there is a general proz $s i$ on of the red-most absorbance maxima of the precursors toward longer wavelengths. Thus the red-most spectral peak of acetone:methanol $(7: 2)$ pigment extracts is a good indication of the last compound synthesized by a given mutant. The pigments identified for each of the strains analyzed here are listed in Table 3.1 .

Extracted pigments from KZR8G9 have absorbance peaks at 718,628 , 572 , and $516 \mathrm{~nm}$ (fig 3.3a, top). The peak at $718 \mathrm{~nm}$ in acetone:methanol suggested a mutations in bchF (Taylor et al. 1983) while the peak at 628 corresponds to the BchB phenotype (Yen and Marrs 1976) (accumulation of PChlide (Biel and Marrs 1983)). Since detailed spectrographic data are not available for $b c h F$ mutants, both the absorbance and fluorescence properties of the pigments accumulated by KZR8G9 were compared to those accumulated by the $b c h F$ point mutant MB1003 (Taylor et al. 1983). An acetone:methanol extract of MB1003 showed a red-most absorbance maximum at $716 \mathrm{~nm}$, 
Table 3.1

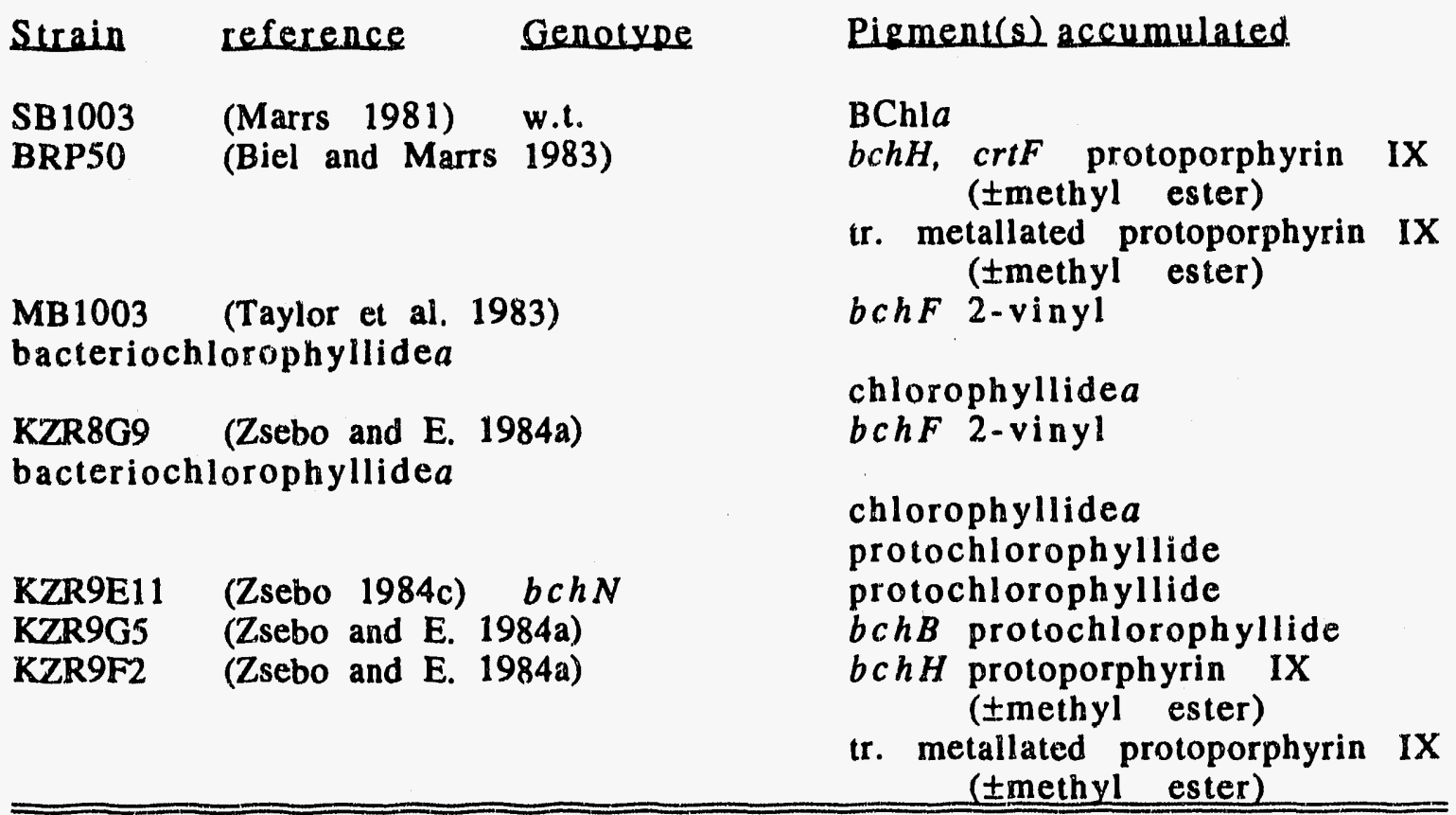

Genotypes and phenotypes of strains used in this study.

consistent with assignment of the lesion in KZR8G9 to $b c h F$, along with additional absorbance peaks at 663,520 , and $488 \mathrm{~nm}$ (fig $3.3 \mathrm{a}$, bottom). Fluorescence emission and excitation spectra of KZR8G9 and MB1003 acetone:methanol extracts showedemission maxima at $740 \mathrm{~nm}$ (fig $3.3 \mathrm{~b}$, inset), for which the excitation maxima were 516 and $718 \mathrm{~nm}$ in both strains (fig 3.3b). This strongly suggests that the same compound, probably 2-vinyl bacteriochlorophyllide $a$ (Pudek and Richards 1975), is the last compound synthesized by each strain, and that KZR8G9 is, indeed, genetically defective in $b c h F$. 

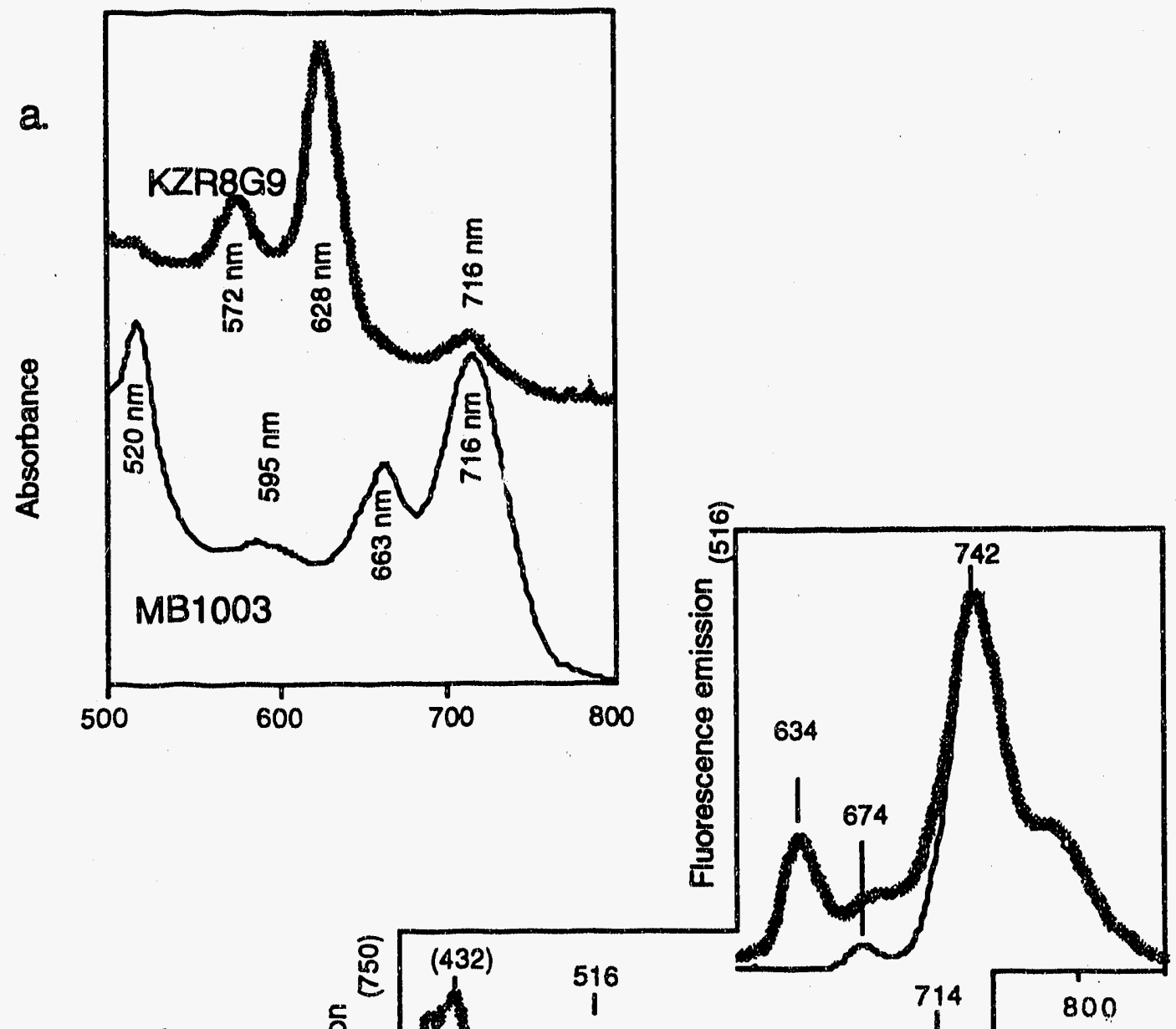

b.

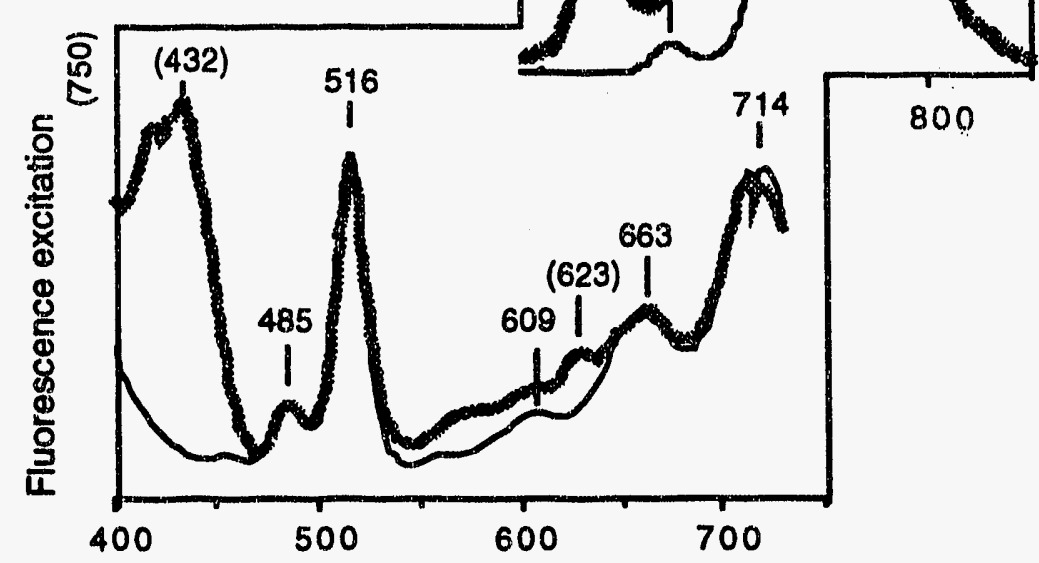

c.

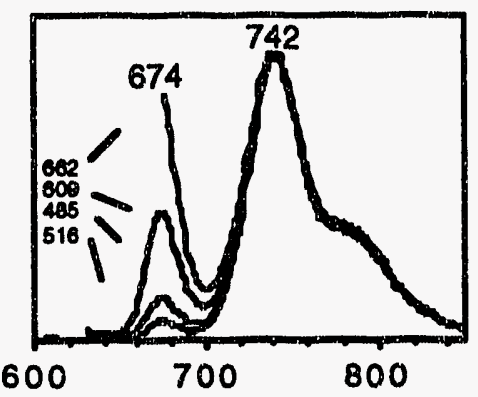


Fig 3.3 Spectra of acetone:methanol (7:2) extracted pigments.

(a) Absorbance spectra of pigments from KZR8G9 (top) and a bchF point mutant, MB1003 (bottom). (b) Fluorescence emission and excitation spectra of KZR8G9 (top, thick line) and MB1003 (bottom, thin line). Excitation was at 516 $\mathrm{nm}$, and emission was monitored at $750 \mathrm{~nm}$. (c) Relative fluorescence emission spectra of MB1003 with excitation at 663, 609, 485, and $516 \mathrm{~nm}$ (top to bottom).

Fig 3.4 Spectra of acetone:methanol $(7: 2)$ extracted pigments. (a) Absorbance spectra of acetone:methanol (7:2) extracted pigment from KZR9G5 and KZR9E11, and fluorescence emission (thin line, exciting at $432 \mathrm{~nm}$ ) and excitation (thick line, monitoring emission at $634 \mathrm{~nm}$ ) spectra of pigments from KZR9G5. (b) Fluorescence emission spectrum of bchH point mutant BRP50, with excitation at $402 \mathrm{~nm}$. 
a.

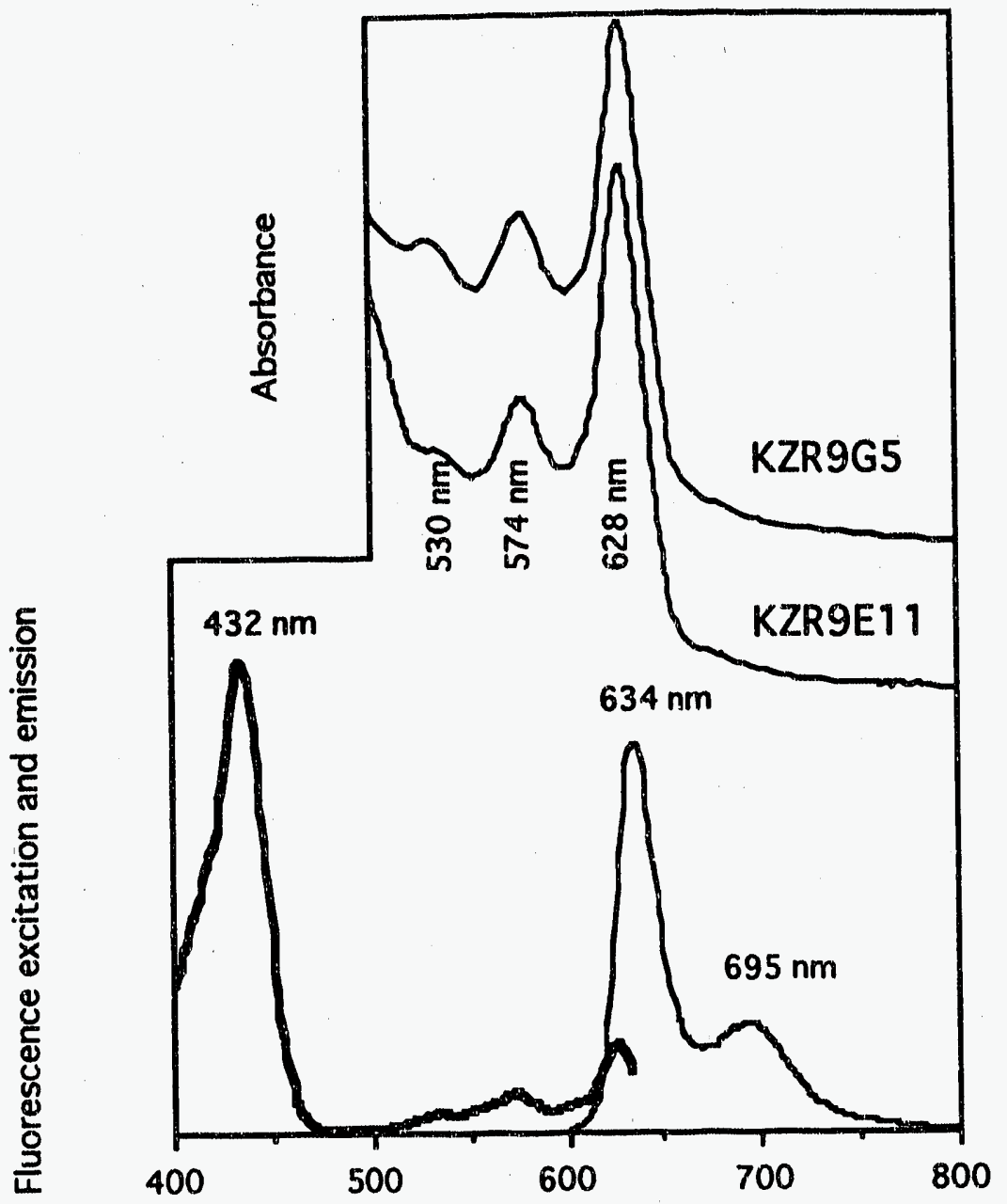

b.

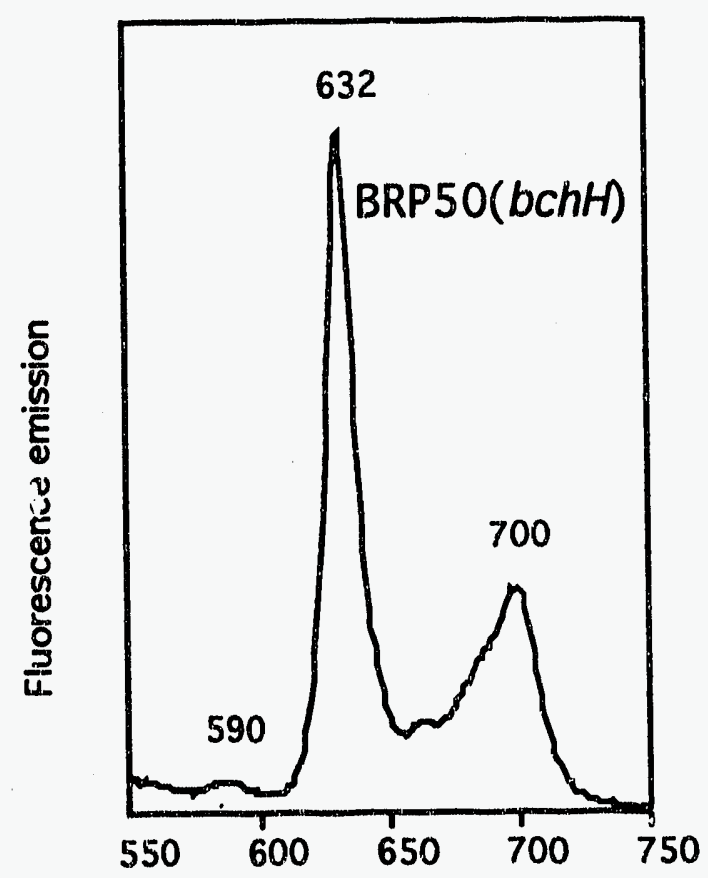


Additional peaks in both excitation spectra (detecting emission at 740 $\mathrm{nm}$ ) are present at 485, 609, and $663 \mathrm{~nm}$ (fig 3.3b). When MB1003 pigment extract was excited at each of these wavelengths, the weak emission peak at 674 $\mathrm{nm}$ was preferentially stimulated, with maximum excitation at $663 \mathrm{~nm}$ (fig 3.3c). All of these data are consistent with the accumulation of chlorophyllide in both MB1003 and KZR8G9 (Goedheer 1966). Finally, there is a strong fluorescence emission from KZR8G9 (but not MB1003) at $634 \mathrm{~nm}$, for which the excitation maximum is at $432 \mathrm{~nm}$ (c.f. fig 3.4, below). Both of these data, as well as the absorbance peak at $628 \mathrm{~nm}$, are consistent with accumulation of PChlide in KZR8G9 (Belanger and Rebeiz 1979, Belanger and Rebeiz 1980).

The mutation carried by the $R$. capsulatus Tn5.7 mutant KZR9G5 defines the bchK locus (Zsebo and E. 1984a). The reported phenotype of KZR9G5 (BChl) is identical to that of point mutants in $b c h H$ (Zsebo and E. 1984a)). However, upon reexamination we find that this strain accumulates a pigment which absorbs maximally at $628 \mathrm{~nm}$ in acetone:methanol (7:2) extracts (fig 3.4a) and $630 \mathrm{~nm}$ in methanol (not shown), which is identical to the BchB phenotype (Yen and Marrs 1976). Furthermore, the fluorescence emission spectrum shows a major peak at $634 \mathrm{~nm}$, with a smaller, broad peak at around $695 \mathrm{~nm}$ (fig 3.4a). The excitation spectra of KZR9G5 detecting at each each of these wavelengths show maxima at 434 and $628 \mathrm{~nm}$ (fig $3.4 \mathrm{a}$ ), demonstrating that both emissions probably arise from the same compound. All of these data are consistent with accumulation of PChlide in strain KZR9G5 (Belanger and Rebeiz 1979, Belanger and Rebeiz 1980). The absorbance spectrum (fig 3.4a) and the fluorescence emission and excitation spectra (not shown) of KZR9E11 are essentially identical to those of KZR9G5, implying that PChlide is also accumulated in KZR9E11. 
R. capsulatus strains KZR9C6 and KZR9F2 both carry transposon Tn5.7 insertion mutations within the fourth open reading frame of BamD (Zsebo and Hearst 1984a). Both of these two and the bchH point mutant BRP50 accumulate a compound which fluoresces maximally at $635 \mathrm{~nm}$ upon excitation at $402 \mathrm{~nm}$ (fig 3.4b). This is indicative of a Mg-less protoporphyrin IX, with or without a methyl ester (Biel and Marrs 1983). There is a second emission peak at $590 \mathrm{~nm}$ with approximately one fiftieth the integrated intensity of the major peak. Emission at this wavelength is likely due to a $\mathrm{Mg}$-containing protoporphyrin, again with or without a methyl ester (Biel and Marrs 1983). Absorbance spectra of all three strains (not shown) are identical to those published previously (Coomber et al. 1990).

The PChlide reductase subunits have homologs in plant chloroplasts.

The bchN gene product shares notable amino acid sequence identity (35.7\%, fig 3.5a) with chlN from Chlamydomonas reinhardtii (Choquet et al. 1992), Marchantia polymorpha (ORF465) (Ohyama et al. 1986), and pine (Lindholm and Gustafsson 1991) chloroplasts,the cyanelle of Cyanophora paradoxa (Stirewalt and Bryant 1991), and with the cyanobacterium Synechosystis PCC6803 (Ogura et al. 1992), bchN and chlL are also homologous with the bchY product of $R$. capsulatus (discussed below). As noted in the introduction, a clear role in PChlide reduction for $c h l N$ has been established genetically (Choquet et al. 1992, Roitgrund and Mets 1990), and so it is not surprising that $b c h N$ shares this function. Given that both $c h l N$ and $b c h N$ share the same enzymatic function, they are most likely true homologs. The $b c h B$ (formerly $b c h K$ ) gene product is $33.7 \%$ identical with that of ORF513 in 


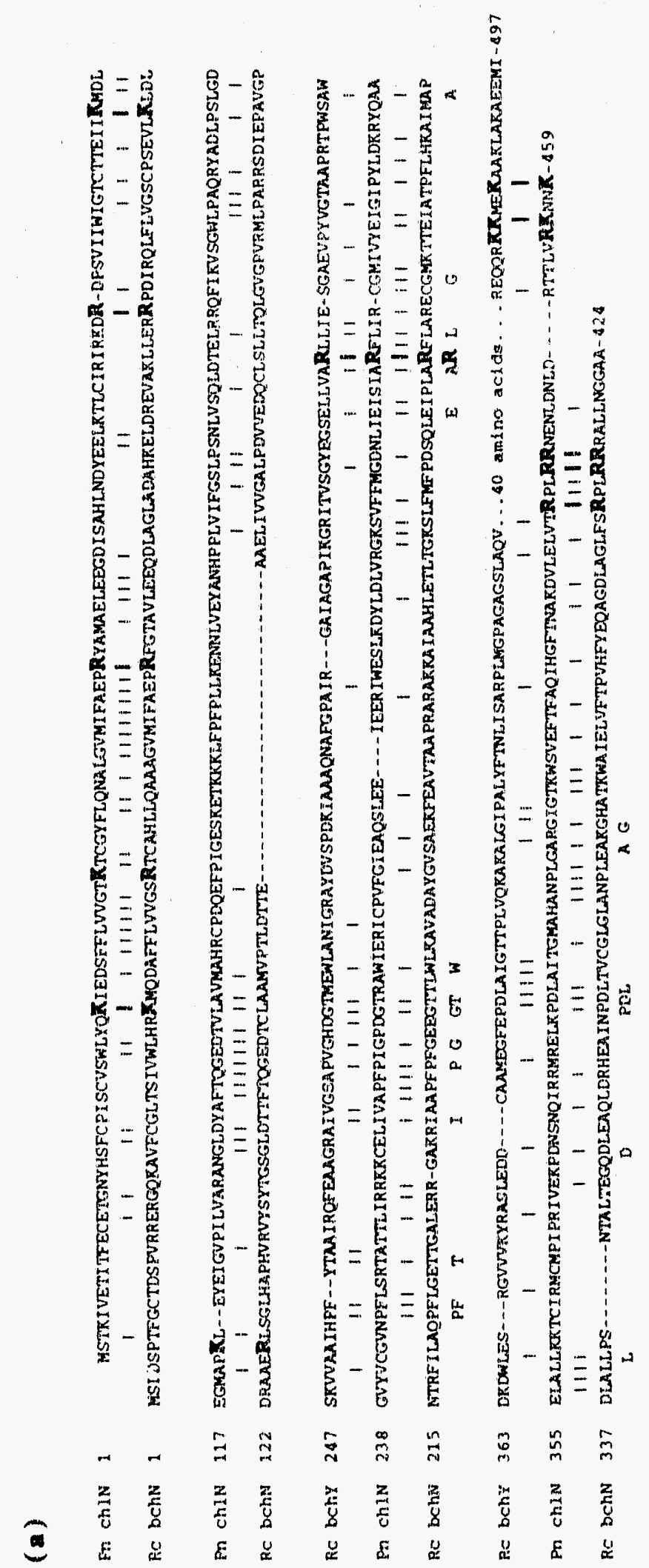



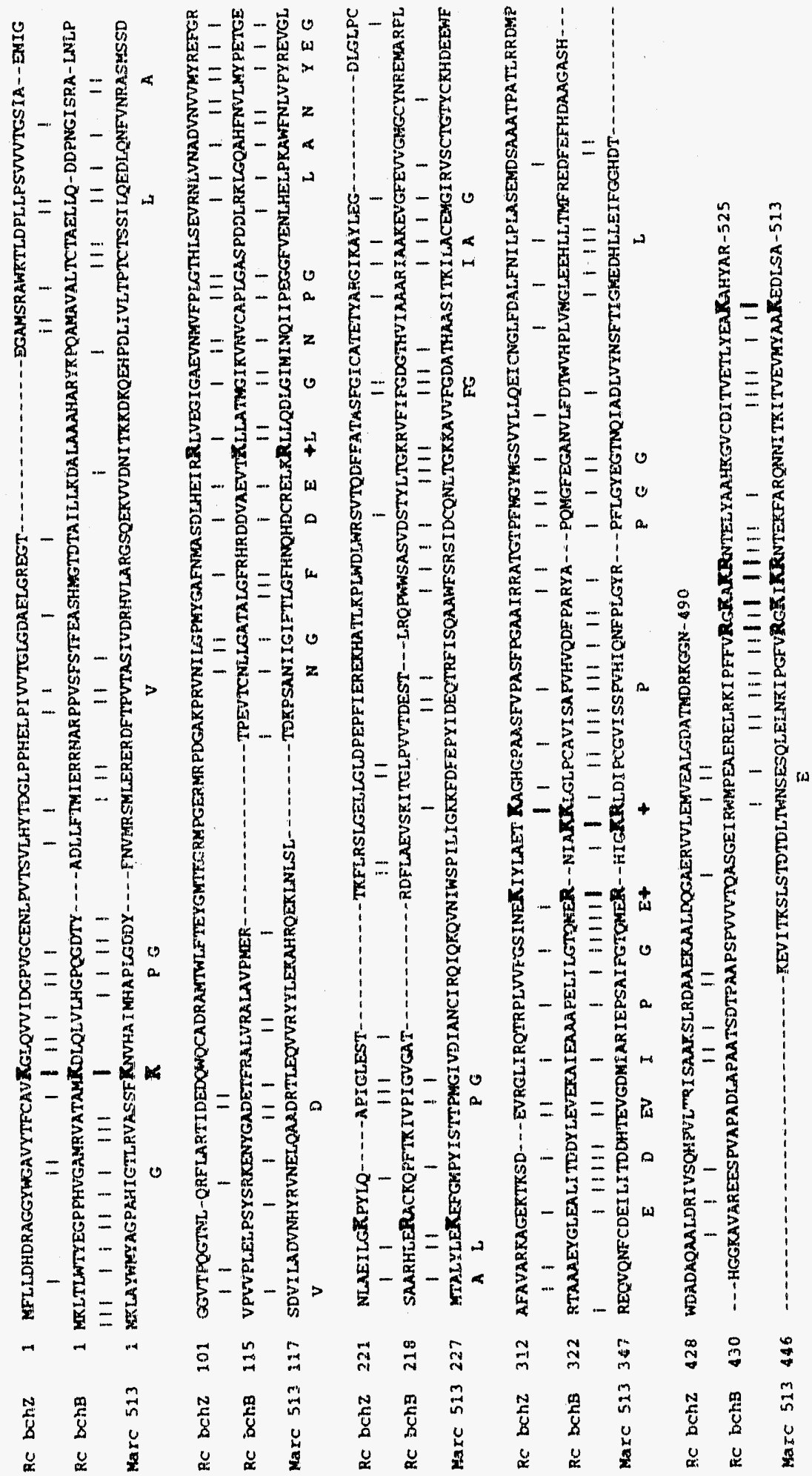

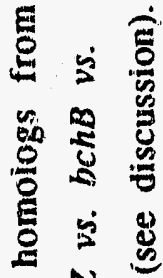

돌 $\frac{1}{8}$

$\div$ e

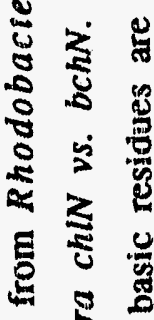

点

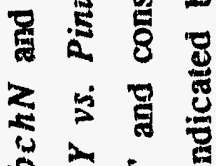

is 2.

楈

䒱

苋

ะ 造递

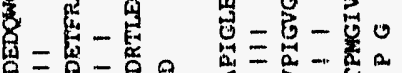

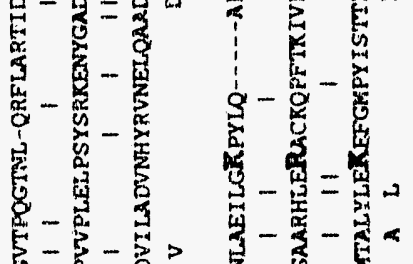

造

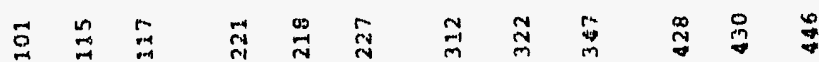

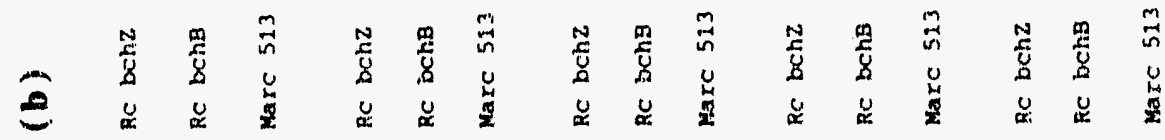

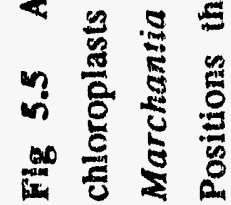


the liverwort chloroplast (fig 3.5b and ref (Burke, Alberti, and Hearst 1991)) and a nearly identical sequence from the $C$. reinhardtii chloroplast (Richard and Bellemare 1990). Considerable sequence identity is also shared with $b c h Z$ from $R$. capsulatus. Like chll and $\operatorname{chlN}$, there is no sequence homologous to ORF513 present in either of the two sequenced angiosperm chloroplasts of rice (Hiratsuka et al. 1989) and tobacco (Shinozaki et al. 1986). The probable role of chlB in PChlide reduction is discussed below.

\section{DISCUSSION}

Operon structure and Gene assignments.

The four genes of BamD appear to form a continuous operon with the first several genes of BamF. In Northern blot experiments using radiolabeled. DNA sequence from the downstream BamF fragment as probe, the measured size of mRNA transcripts placed the $5^{\prime}$ end of the message near the upstream end of BamD (Bauer et al. 1991a, Yang and Bauer 1990). Deletion experiments have also shown that the ptomoter for bchL and $b c h M$, both of which are in BamF, is upstream of one or both of the two Xhol sites in BamD (Yang and Bauer 1990). We find recognizable promoters only upstrean of the first ORF, and the coding segments of all four genes overlap with each other by at least one nucleotide. Both observations argue for a single, polycistronic message. It is therefore most likely that all of the genes in BamD are part of the same operon: bchFNBHLMorfl695 (fig 3.6). (The name orfl695 refers to the position of the AUG etart codon relative to the BamHI site dividing BamD from BamF.

Since bchL contains one fewernucleotide than was originally reported (M.Alberti, unpublished observation and ref (Yang and Bauer 1990)), this AUG is at position 1695.) 


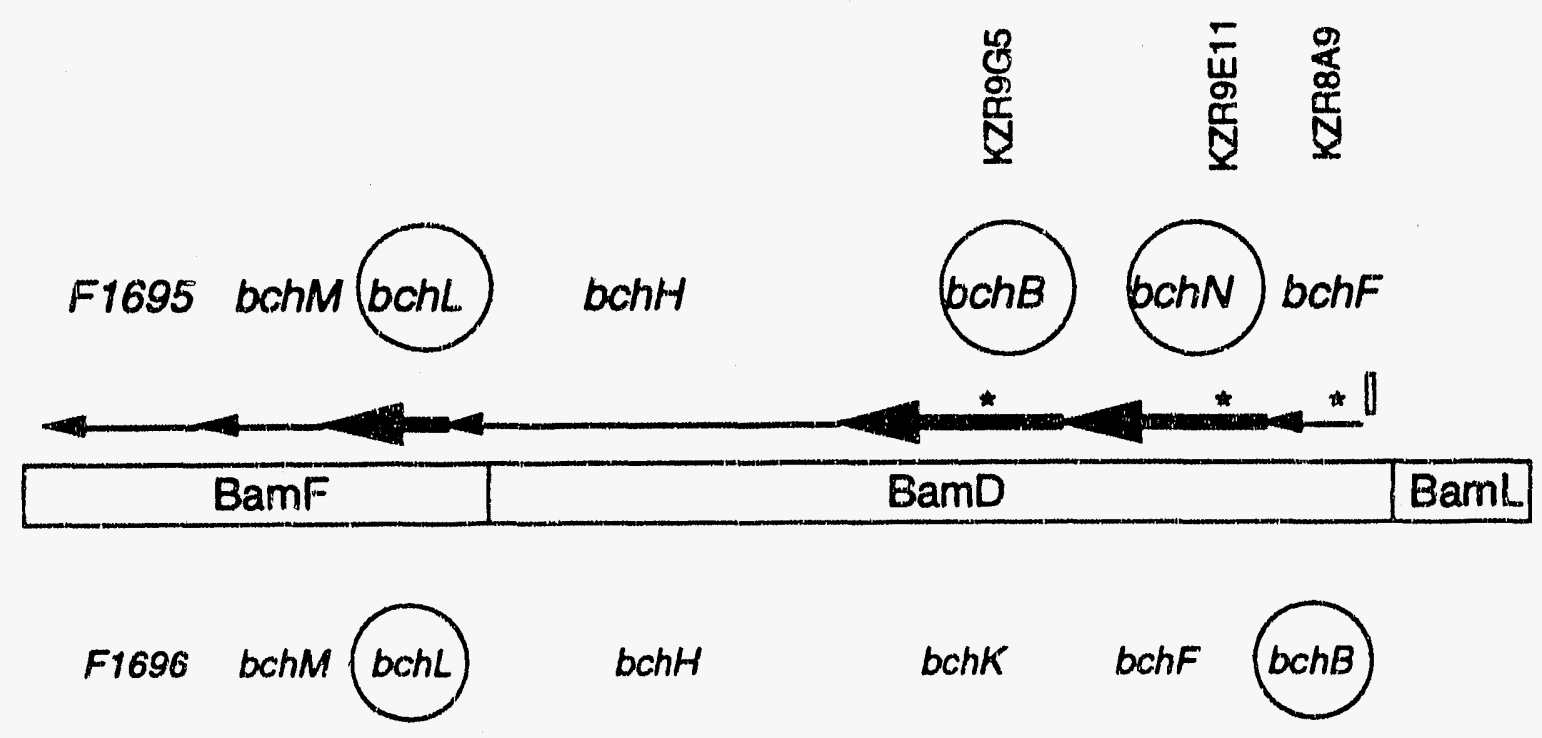

Fig 3.6 Revised gene map of the bchFNBHLMorfl695 operon spanning the BamD and BamF fragments. The gene order supported by this work is given above the line, while that used in previous maps is given below the line. PChlide reductase subunit genes are circled and shown with bold arrows. BamHI sites are indicated by vertical lines with open circles. The locations of the three transposons mapped in this study are indicated by "*" while the location of the putative promoter sequence are shown by the small vertical box.

The transposon in KZR9G5 is located within the third ORF of BamD. A pRPS404 derivative carrying Tn5.7 insertion 9G5 is able to complement bchH point mutants (Zsebo and E. 1984a), and so the lesion is nonpolar with respect to $b c h H$. Therefore, the accumulation of PChlide (BchB phenotype) by KZR9G5 is due to disruption of the third ORF of BamD. This complementation was 
originally used to establish the existence of the $b c h K$ gene (Zsebo and $\mathrm{E}$. 1984a), although the reported BchK phenotype is indistinguishable from the BchH phenotype. There is a bchH Tn5.7 insertion mutation (9F2) which was reported as giving rise to the phenoiype reported here for KZR9G5. In all likeiihood, the errant report of a "BchK phenotype" for KZR9G5 and a BchB phenotype for KZR9F2 represent the switching of their respective labels at some point in the original analysis. There are several transposon insertion mutants in the related bacterium $R$. sphaeroides which have been assigned as lesions in $b c h K$ because they give rise to the $\mathrm{BchK}\left(\mathrm{BChl}^{-}\right)$phenotype and are located in approximately the same position (Coomber et al. 1990). However, no complementation experiments have been done with these strains to exclude polar inactivation of the downstream $b c h H$ gene. In order to avoid similar confusion in the future with the previously described (but misleading) BchK phenotype, we refer to the third ORF of BamD as $b c h B$.

Strain KZR9E11 also accumulates PChlide, and it carries a Tn5.7 insertion in the second ORF of BamD. The mutated gene was originally refered to as bchN (Zsebo 1984c), but was not included in the early genetic maps because no complementation had been done either with this strain or with the R-prime plasmid carrying the transposon. Nevertheless, it is unlikely that the phenotype of KZR9E11 is due to polar inactivation of $b c h E$, because a polar mutation $\mathrm{k}$ suld also be expected to inactivate $b c h H$. We therefore conclude that $b c h N$ is also required for PChlide reduction. The similarities between $b c h N$ and $c h l N$ (see below) also support a role for $b c h N$ in PChlide reduction.

KZR8G9 was originally reported to accumulate P630 (Zsebo and E. 1984a), which would have made it yet another subunit of PChlide reductase. Indeed, 
the major atsorbance peak is at $630 \mathrm{~nm}$. Unlike the two strains above, however, some of the pigment in KZR8G9 is able to proceed further in the pathway until reaching a block at the hydration of the 2-vinyl group to produce a BchF phenotype. The pRPS404 derivative carrying Tn5.7 insertion $8 G 9$ fails to complement the bchF point mutant MB1003 (Zsebo and E. 1984a), offering genetic support to the assignment of this ORF as bchF in addition to the spectral data presented here. The same plasmid does, however, complement the bchB insertion mutant KZR9G5 (Zsebo and E. 1984a), demonstrating that the mutation is not strictly polar.

Both $b c h F$ mutants accumulate additional compounds besides 2-vinylbacteriochlorophyllide $a$. Chlorophyllide accumulation in each strain may be due to hindrance of the chlorin reductase as a result of the large buildup of its product, the substrate for the $B c h F$ gene product. The large accumulation of PCnlide in KZR8G9, a feature which is absent in spectra of MB1003, suggests that readthrough of downstream genes is sufficient to sustain photosynthetic growth in complementation assays, but not sufficient to avoid a partial block of PChlide reduction by $b c h N, b \operatorname{chB}$, and $b c h L$.

The third subunit of light-independent PChlide reductase in plants.

Marchantia chloroplast ORF513 shares notable amino acid identity with $b c h B$ and with the chlorin reductase subunit $b c h Z$. No function has been assigned experimentally to ORF513, but there are several lines of evidence which suggest that it is part of the chloroplast's light-independent PChlide reductase. First, the $y-1$ nuclear mutant of $C$. reinhardtii is deficient in dark PChlide reduction, and so $y-l$ cultures grown aerobically in the dark are 
yellow in appearance (Wang 1979). Upon illumination, these cultures green rapidly via light-dependent PChlide reduction, even if chloramphenicol is added to the culture prior to illumination to block protein synthesis within the chloroplast (Gershoni et al. 1982). This "CAP greening" demonstrated that no light-dependent chlorophyll synthesis function requires translation within the chloroplast. For years the accepted dogma as a result of these experiments has been that all Chl-synthesis genes are nuclear encoded, dogma which was shattered by the discovery of the chloroplast-encoded $\operatorname{chlN}$ (gidA) gene (Roitgrund and Mets 1990). On the other hand, it would still have been possible that some other required, light-independent Chl-synthesis function, such as the $\mathrm{Mg}$ chelatase, could be redundantly encoded both in the chloroplast and in the nucleus. However, when wild-type $C$. reinhardtii is grown in the presence of sublethal concentrations of CAP in the dark, large amounts of PChlide accumulate which is rapidly converted to Chl upon illumination (Roitgrund 1989). Clearly the only step in Chl synthesis which is dependent upon chloroplast-encoded products in thai of light-independent PChlide reduction. Any chloroplast genes homologous to $R$. capsulatus $\mathrm{BChl}$ synthesis genes are therefore expected to be involved in PChlide reduction. From this line of reasoning and from the strong amino acid sequence conservation between $b c h B$ and ORF513, we propose that ORF513 is the third subunit of the lightindependent PChlicie reductase in chlorophyllous organisms, along with the other two subunits chlL and $\operatorname{chlN}$. Its role in the enzyme complex is likely exactly analogous to that of $b \operatorname{chB}$ and closely related to that of $b \operatorname{chZ}$ (see next section) in the chlorin reductase. By extension, we refer to ORF513 and its homologs as chlB. 


\section{Properties of PChlide reductase.}

$b c h B$ and chlB (ORF513) can both be aligned with $b c h Z$ from $R$.

capsulatus (Burke, Alberti, and Hearst 1992a) (fig 3.5b), which is required for chiorin ring reduction (Young et al. 1989). The residues that are conserved between bchz and each of the other two appear to play an important role in all three proteins, since more than half $(54 \%)$ of all such two-way matches are shared by all three proteins. Although a complete statistical analysis of this arrangement of matches is beyond the scope of this communication, we consider it unlikely that so many three-way matches would occur by chance. The similarity between $b \operatorname{ch} Z$ and $b \operatorname{ch} B-\operatorname{chlB}$ is especially in evidence between residues $160-230$ and $330-365$ in the $b \operatorname{ch} B$ sequence. In these two regions, one quarter of all residues are conserved among all three proteins.

Our findings support the evolutionary relatedness of the enzyme complexes for light-independent PChlide and Chlide reduction. $b c h X, b c h L$, and chlL are clearly derived from a common ancestral Chl Fe protein (Burke, Alberti, and Hearst 1991, Burke, Alberti, and Hearst 1992a). It is now also likely that $b c h Z, b c h B$, and $c h l B$ similarly share a common ancestor. If $b c h N / c h l N$ has a functional counterpart in the $b c h X Y Z$ system, it is likely to be $b \operatorname{ch} Y$, simply by elimination. The midsection and C-terminus of the bchY product can be readily aligned with $b c h N-c h l N$, as shown in fig 3.5a, and there is a significant number of three-way matches. The N-terminus, however, can be aligned in each of several different ways depending on whether matches with $\operatorname{chlN}$ or $b \operatorname{ch} N$ are given priority, and so this region is not included in the alignment. Here any similarities between bchY and the other two have become muddied over the time since their divergence. 
One of the predictions for light-independent ring-rcducing enzymes, based on the strong similarities of $\mathrm{Chl} F \mathrm{Fe}$ proteins with nitrogenast $\mathrm{Fe}$ protein, is that acidic residues between the two Fe-binding Cys residues on the Fe proteins mediate ionic interactions with the ancillary proteins (Burke, Alberti, and Hearst 1992a, Willing and Howard 1990). Conserved basic residues in at least one of the two groups of ancillary proteins may interact with these acidic residues on the $\mathrm{Chl} F$ Ar $y$ is found in all three sequences in the alignment of $b c h Z, b c h B$, and $\operatorname{ch} / B$, while the sequence $\operatorname{RGK}(A / I) K R$ is shared by $b \operatorname{ch} B$ and $c h l B$ in the C-terminal extension of these two relative to $b c h Z$ (fig 3.5b). A similar analysis of $b c h N$. chlN is more difficult, since a good alignment with bch $Y$ is not yet possible. There are, nevertheless, a number of basic shared residues between $b c h N$ and chlN, including the sequence RPLRR near the C-terminus, and at least one Arg residue is shared by all three (fig $3.5 \mathrm{a}$ ).

In vitro PChlide reduction has recently been observed to occur independently of light in preparations from the cyanobacterium Anacystis nidulans (Peschek et al. 1989a, Peschek et al. 1989b). Because chlL sequences have previously been detected in cyanobacteria (Burke, Alberti, and Hearst 1991, Fujita et al. 1991), we posit that the enzyme activity observed by Peschek et al. arises from a $\operatorname{chlL}-N-B$ enzyme system. Since these authors observed their activity in sucrose-density gradient purified membrane fractions, we examined the sequences of $b \operatorname{chL}, \operatorname{chlL}, b \operatorname{ch} N, \operatorname{chlN}, b \operatorname{ch} B$, and $\operatorname{chlB}$ for hydrophobic sequences. The strongest indication of hydrophobicity was found in sequences shared among all of the Fe proteins. These sequences are buried within the homodimer of the nifH protein in its crystal structure (Rees 1991), however, and do not represent trans- membrane helices. There are 
between one and five weakly hydrophobic stretches in each of the four remaining sequences (not shown). However, none of these can be assigned unambiguously as membrane-spanning domains, nor do the most prominent peaks in each hydrophobicity plot fall at the same place in their respective alignments. The in vitro reductions observed by Peschek et al. were dependent on NADPH, and were reversed by substitution of NADP+. Nevertheless, we find no evidence of a binding site for ADP, as judged by the criteria of Wierenga, Terpstra, and Hol (1986), in any of $b \operatorname{chN}, \operatorname{chlN}, b \operatorname{ch} Y$, $b \operatorname{ch} B, \operatorname{chl} l B$, or $b \operatorname{ch} Z$. Future detailed biochemical characterization of both the chlorin and PChlide reductase systems may offer insight into the relative roles of each of the subunits and their relationships to one another.

The role of $b c h H$.

The exact enzymatic function of the $b c h H$ product has not been well established. The bulk of the evidence suggests that $b c h H$ encodes the methyltransferase, although the debate is probably far from over. bchH point mutants are blocked prior to $\mathrm{Mg}$ chelation and methylation of the C-13 propionic acid on ring $D$ (Biel and Marrs 1983). As has been discussed elsewhere (Biel and Marrs 1983, Gorchein 1972, Gorchein 1973), these two steps appear to be obligate'y coupled in $R$. capsulatus, and no mutants have been observed to accumulate large amounts of $\mathrm{Mg}$-protoporphyrin IX. The bchH mutants we have studied retain some $\mathrm{Mg}$ chelatase activity, as shown by the fluorescence emission peak at $590 \mathrm{~nm}$. The fraction of protoporphyrin that contains $\mathrm{Mg}$, however, is so low that it is in no way clear where the activity is coming from; i.e., whether the $\mathrm{Mg}$ chelatase is coded by some other gene(s) that require an intact $b c h H$ product for complete activity, or whether it is coded by $b c h H$ and is not completely blocked by the lesions. There is a 


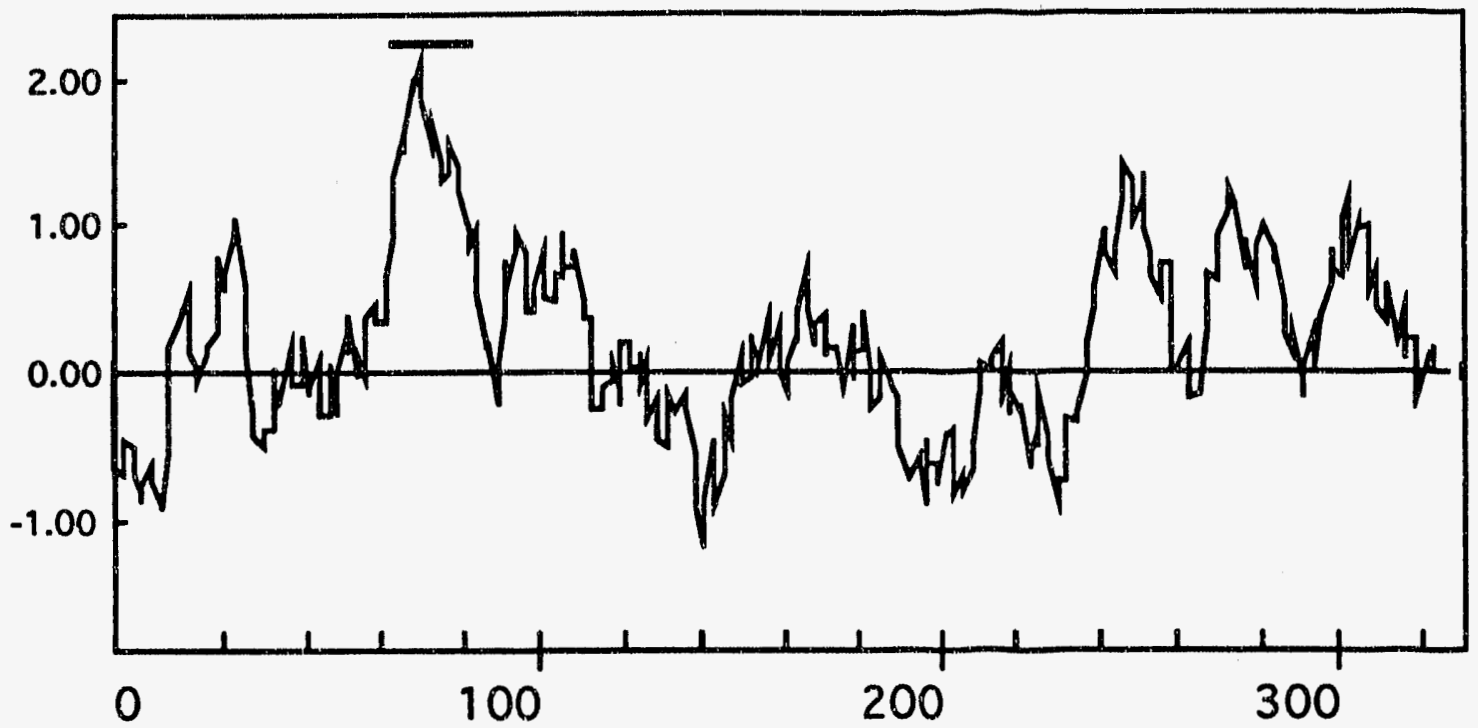

Fig 3.7 Hydrophobicity plot of the N-terminal 1/3 of the $B, h H$ product. The bar over the graph is the length of 20 amino acids.

potential membrane-spanning region near the N-terminus of the $b c h H$ protein which could serve to anchor the protein to the membrane, (fig 3.7) but since both $\mathrm{Mg}$ protoporphyrin IX methyltransferase (Hinchigeri, Nelson, and Richards 1984) and Mg-chelatase (Walker and Weinstein 199:) activities are reported to be membrane associated, this observation would be consistent with a role for $b c h H$ in either function.

At one time there were four candidate genes for the two enzymes $\mathbf{M g}$ chelatase and methyltransferase: $b c h D, b c h H, b c h l$, and $b c h K$ (Zsebo and $\mathrm{E}$. 1984a). We have shown here that the " $b c h K$ " gene product is required for PChlide reduction, and we present, evidence elsewhere that the bchl gene produit is involved in $\mathrm{Mg}$ chelation (Armstrong et al. 1992c). This leaves 
bchD, which is coexpressed with bchl (Armstrong et al. 1992c), and bchH without definite functions. $\mathrm{Mg}$ chelatase from plants can be separated into two components, one membrane-bound and one soluble (Walker and Weinstein 1991). Either bchD or bchH is probably the second component of $\mathrm{Mg}$ chelatase. When S-adenosyl methionine (SAM) and protoporphyrin IX are added to membrane preparations from $b c h D$ and $b c h H$ transposon mutants of $R$. sphaeroides, the bchH mutants were unable to convert the substrate to the methyl ester, while the $b c h D$ mutant retained this activity (Richar's et al. 1991). The most likely interpretation of these data is that bchH encodes the methyltransferase. Positive demonstration of methyltransferase activity corresponding to the $b c h H$ gene product would allow an unambiguous assignment of methyltransferase function to $b c h H$. Preliminary work in our lab with $b c h H$ protein expressed in $E$. coli has not been successful in obtaining methyltransferase (MT) activity in vitro (Hundle 1991). This may be due to an inherent defect in the in vitro system, or it may simply reflect a need for the presence of the $b c h D$ and $b c h l$ gene products in an obligately coupled reaction (Biel and Marrs 1983, Gorchein 1972, Gorchein 1973). 


\section{Chapter 4: The early evolution of photosynthesis and nitrogen fixation.}

\section{ABSTRACT}

Chlorophyll (Chi) is presently viewed as having preceded bacteriochlorophyll (BChl) as the primary photoreceptor pigment in early Fhotosynthetic systems. The recent elucidation of the isequenises of Chl- and BChl- ayntiesizing enzymes, coupled with expanding lknowledge of bacterial phylogeny and the properties of photosyathesizers whin etach bacterial division, has forced a reavaluation of this position. In particular, the enzyme that reduces pheoporphyrins 20 chlorins shares many properties with the enzyme that reduces chlorins to bacteriochlorins. Bith are three-subunit enzymes in which each subunlt from one reductase system shares significant amino acid identity with a subunit in the other system. The two reductases are therefore likely to be homologous. Because of the much broader distribution of $\mathrm{BCH}$ than $\mathrm{Chl}$ in photosynthetic reaction centers, I propose that the ancestor of PChlide reductase and chlorin reductases performed both reactions, and that $\mathrm{BCh}$ became established as reaction center chromophore before Chl. Of special interest are the so-called "Chlorophyll Fe protein" subunits of each reductase. These appear to serve as the unique electron donors to their respective catalytic subunits. The Chlorophyll Fe proteins not only share amino acid sequence Identity with each other, but also with the nitrogenase $\mathrm{Fe}$ protein nifH. In detailed phylogenetic analyses of these three classes of iron proteins (bchL-like, bchX-like, and nifH-like) "the $\mathrm{Chl} F$ proteins group together. Using the nifH proteins as outgroup, the Chl Fe protein tree is rooted on the $b c h X$ (chlorin reductase) lineage. This rooting strongly suggests that the common ancestor of cyanobacteria and purple 
bacteria used $\mathrm{BChl}$ in its reaction center, and that Chl-containing reaction centers were a later invention unique to the cyanobacteria/chloroplast lineage.

\section{INTRODUCTION}

Photosynthesis may be nearly as old as life itself. Some have speculated that photochemical enezgy transduction may have actually preceded life as we know it (King 199C, Masinovsk'y et ul. 1989, Mauzerall 1990, Morowitz, Heinz, and Deamer 1988). Fossil evidence for ancient photosynthetic cellular life date back at least $3.5 \mathrm{~Gy}$ to the stromatolites from the Warrawoona Group of Wester: Australia. The microfossils in these stomatolites have a cellular morphology similar to modern cyanobacteria, and the stratifications show evidence of a complex microbial ecosystem similar to those found in modern benthic microbial mats (Schopf 1992. Schopf and Packer 1987). Th. Isua Supracrustal Belt of southwestern Greenland was laid down a few tusndred million years before, around 3.7-3.8 Gya. These deposits retain the signature of having been derived from biological carbon fixation (Hayes, Kaplan. and Wedeking 1983a, Schidlowski 1988, Schidlowski, Hayes, and Kaplan 1983), In particular, a significant portion of the deposits are enriched in ${ }^{12} \mathrm{C}$ over ${ }^{13} \mathrm{C}$ relative to the distribution of these isotopes found in nonbiologically derived carbon deposits (carbonates). The segree of enrichment is consistent with its having been fixed from atmospheric $\mathrm{CO}_{2}$ by an enzyme with carbon isotopic kinetic fractionation properties similar to those of ribulose (1,5)-bisphosphate carboxylase-oxygenase (RuBisCO), followed by partial reequilibration at high temperatures and pressures in formations containing both biologically and nonbiologically derived carbon (Schidlowski 1988). The Isua deposits are therefore often refered to as the earliest geochemical evidence of ancient 
photosynthetic processes, although the alterations of these deposits over time open the door to numerous alternative interpretations. The upper limit on the origin of life is the interval from 4.2 to 4.0 billion years ago, at the end of the bombardment of Earth by large asteroides (Joyce 1991). This leaves a window of 200-600 million years during which recognizable photosynthetic cellular systems became established. It is mostly this time period that $I$ address in this chapter.

Chlorophyll (Chl) and bacteriochlorophyll (BChl) are the photochemically active reaction center pigments for all extant photosynthetic organisms except for the extreme halophilic Archacbacteria. These latter bacteria use a carotenoid photopigment in place of a tetrapyrrole, and pump protons directly through bacteriorhodopsin proton pumps instead of cycling them through quinones or other carrier compounds (Olson and Pierson 1987). Their bacteriorhodopsin system is unrelated to (bacterio)chlorophyllcontaining photosystems, and is therefore omitted from further discussion here. We focus our attention on the reaction center pigments to the exclusion of phocopigments used in light-harvesting antennae for the simple reason that the primary charge separation events that are the heart of photosynthesis occur within the reaction center. As such, the reaction center is absolutely central to the conversion of light energy into chemical energy. A reaction center without an antennae can support photosynthetic growth in many organisms (see, for example, references in (Coleman and Youvan 1990)), wherras an aricina without a reaction center cannot.

During the synthesis of $\mathrm{Chl}$ and $\mathrm{BChl}$, one reduction of the tetrapyrrole ring system converts pheoporphyrins into chlorins, while a second ring 
reduction converts inlorins into bacteriochlorins (fig 4.1). Chl can be made directly from ch'orophillide (a chlorin) by esterification of the free propionic acid group, whereas chlorophillide must first be reduced to a bacteriochlorin prior to esierification in the synthesis of BChl. The recapitulation theory, often summed up in the aphorism. "Metabolism recapitulates evolution," holds that modern metabolic pathways evolved from the beginning toward the end. Compounds with shorter biosynthetic pathways are thought to be more reflective of the ancestral state than compounds which require numerous additional modifications. By this logic, Chl has been presumed to have: preceded $\mathrm{BChl}$ in ancient photosynthetic organisms (Granick 1965, Olson and Pierson 1987). Only later, when some organism acquired the ability to make BChl, was it able to take advantage of the longer wavelengths that penetrate

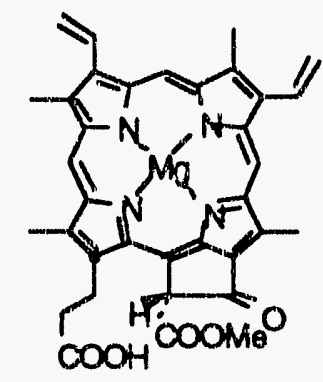

$\lambda_{\max }=628 \mathrm{~nm}$
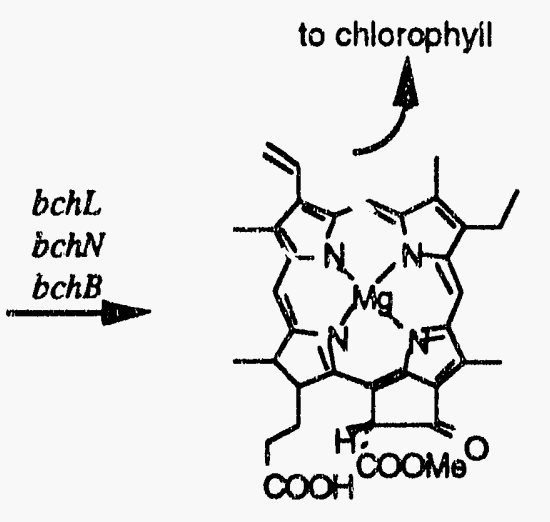

$$
\lambda_{\max }=656 \mathrm{~nm}
$$
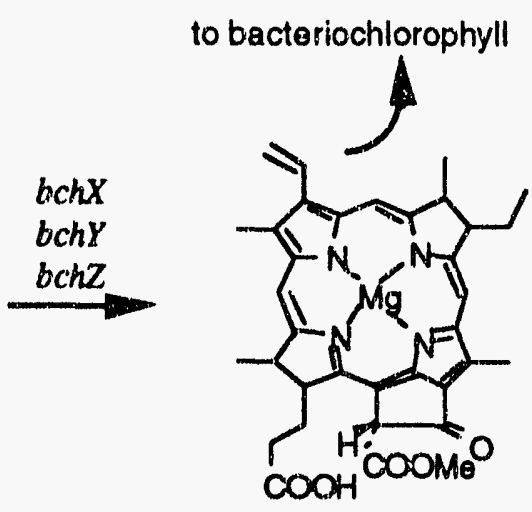

$\lambda_{\max }=718 \mathrm{~nm}$

fig 4.1 The reduction reactions of $\mathrm{Chl}$ and $\mathrm{BChl}$ synthesis, from protochlorophyllide (PChlide) to chlorophyllide to a bacteriochlorophyllide. The $\mathrm{C}-17$ atom of PChlide is highlighted. In Chlc, the propionate at this position is replaced by acrylate. 
deep into the water column. The "Chl-first" application of the recapitulation theory has become known as the "Granick hypothesis," in honor of its first expositor (Granick 1965).

The question of whether $\mathrm{Chl}$ or $\mathrm{BChl}$ appeared first in early photosynthetic systems is fundamentally an issue of whether the first pheoporphyrin reductase performed one or both reductions of the macrocyclic ring. Therefore, I begin by summarizing recent molecular genetic data revealing the numerous similarities between the protochlorophyllide and chlorin reductases from several distantly related photosynthetic bacteria. Further evidence is drawn from the phylogenetic distribution of $\mathrm{Chl}$ and $\mathrm{BChl}$. A quantitative phylogenetic analysis of subunits from each class of protein add strong support to the antiquity of BChl. Next, I expand the discussion to analyze the evolutionary significance of the similarities between the nitrogenase iron protein (nifH) and the putative iron proteins in each of the two pigment reductase systems. Finally, I examine the question of what photopigments may have been used in ancient reaction centers prior to the introduction of the modern pigments $\mathrm{BChl}$ and $\mathrm{CH}$, which I refer to collectively on occasion as $(B) \mathrm{Chl}$.

\section{Chlorophyll vs. Bacteriochlorophyll}

The immediate biosynthetic precursor to $\mathrm{Chl}$ in modern biochemical pathways is the pheoporphyrin protochlorophyllide (PChlide). The chemistry of ring $D$ reduction by $P C h l i d e$ reductase is nearly identical to that of ring $B$ reduction by chlorin reductase. In 'each case, the outer double bond of a fivemembered ring flanked by two other conjugated rings is reduced to a single 
bond. The similarity of the two reactions is underscored by the similarities of the enzymes responsible for the two steps, both of which require three subunits for activity. PChlide reductase is encoded by three genes, refered to as $b c h L, b c h N$, an.: bchB (Burke, Alberti, and Hearst 1992b), in R. capsulatus and by $\operatorname{chlL}, \operatorname{chlN}$, and $c h l B$ in cyanobacteria and most plant chloroplasts (except angiosperms) (Burke, Alberti, and Hearst 1992b, Choquet et al. 1992, Fujita el al. 1992, Roitgrund and Mets 1990, Stirewalt and Bryant 1991, Suzuki and Bauer 1992) Chlorin reductase is codea by $b c h X, b c h Y$, and $b c h Z$ in both $R$. capsulatus (Burke, Alberti, and Hearst 1992a) and the closely related $R$. sphaeroides (McGlynn and Hunter 1992). Each subunit from one reductase shares significant amino acid identity with a subunit of the other reductase. The percent identities in the $b \operatorname{ch} L(\operatorname{chl} l L)-b \operatorname{chX}, b \operatorname{ch} N(\operatorname{chlN})-b \operatorname{ch} Y$, and $b c h B(\operatorname{chlB})-b \operatorname{ch} Z$ alignments are given in table 4.1. All three PChlide

Table 4.1.

\begin{tabular}{|c|c|c|c|c|c|c|}
\hline & Ch/L & $\operatorname{ch} / N^{a}$ & $\operatorname{ch} / B^{a}$ & bchl & $b c h N$ & $b c h B$ \\
\hline bchL & $50 \%$ & & & & & \\
\hline$b c h N$ & & $36 \%$ & & & & \\
\hline$b c h B$ & & & $34 \%$ & & & \\
\hline bch $x$ & $32 \%$ & & & $34 \%$ & & \\
\hline bchY & & $<20 \%$ b & & & $22 \% b$ & \\
\hline$b c h Z$ & & & $20 \%$ & & & $24 \%$ \\
\hline
\end{tabular}

Relative similarites of the PChlide reductase and Chlorin reductase subunits. Only homolgous subunits are compared.

a. These genes have also been refered to under other names: chIL = frxC or gidB; $\operatorname{chIN}=$ ORF 465 or gidA; chlB $=$ ORF510

b. These alignments are sufficiently uncertain that exact \% identities cannot be had with certainty. 
reductase genes from $R$. capsulatus are more similar to their PChlide reductase homologs in cyanobacteria and chloroplasts than they are to the respective subunits of the chlorin reductase. In fact, the significance of the alignments of $b c h Z$ with $b c h B-c h l B$ and of $b c h Y$ with $b c h N-c h l N$ is only made apparent in three-way alignments (see, for example, figs $3.5 \mathrm{a}$ and $\mathrm{b}$ in chapter 3 ), where a large number of residues are found to be conserved among all three proteins within each grouping (Burke, Alberti, and Hearst 1992b). Given the similarities of function and amino acid sequences, the respective pairs of enzyme subunits most likely share common ancestry (i.e., they are homologous).

Alignment of the $b c h X, b c h L$, and $c h l L$ subunits (see fig 2.7 in chapter 2) provides convincing evidence that the PChlide and chlorin reductases are derived from a common ancestral reductase system, as well as offering insight into the nature of these proteins. The amino acid similarities observed among these proteins are also shared with the nitrogenase Fe protein, nifH, and they are strongest in sequences known to bind $\mathrm{MgATP}$ and a [4Fe4S] cluster. The pigment synthesis enzymes are believed to also bind a [4Fe4S] cluster between two subunits of a homodimer (thus they are refered to collectively as the Chl Fe proteins) and to utilize ATP hydrolysis as part of their enzymatic mechanism in serving as unique electron donors into the other two subunits, where catalysis takes place (Burke, Alberti, and Hearst 1991, Burke, Alberti, and Hearst 1992a, Fujita et al. 1989). Dimerization and apparent ATP binding have been demonstrated experimentally for the chlL product from the Marchantia polymorpha chloroplast (Fujita et al. 1989), but there has not yet been any direct demonstration of $\mathrm{Fe}: \mathrm{S}$ binding, nor has any biochemical characterization of the $B c h L$ or $B c h X$ gene products been carried out. Nearly 
all of the present notions about these Fe protein subunits comes from their alignment with nifH and from the extensive biochemical and structural characterization that has been done with the nitrogenase $\mathrm{Fe}$ protein. The three-dimensional locations of the conserved amino acids supports the structural similarities among these $\mathrm{Fe}$ proteins, including the dimeric nature of each. From a detailed analysis of the structural and mechanistic implications of these alignments (Burke, Alberti, and Hearst 1992a), it is clear that $c h l L, b c h L, b c h X$, and $n i f H$ fold into similar three-dimensional structures, perform largely the same electron transfer functions (albeit to different ends), and have evolved under similar structural constraints.

Evidence for the common ancestry of the two enzyme systems may also be preserved in the arrangements of their respective genes, both in purple bacteria and in chloroplasts. The three genes of the chlorin reductase of $R$. capsulatus are arranged sequentially: $b \operatorname{chX}, b \operatorname{ch} Y, b \operatorname{ch} Z$. If this was the ancestral arrangement (fig 4.2a), it would have fasilitated the simultaneous duplication of all three to form the $b \operatorname{ch} L, b c h N, b \operatorname{chB}$ system (fig 4.2b). This gene order may be preserved in some organism yet to be studied, but not in any examined thus far. Instead, one of two rearrangements has taken place: In Rhodobacter, bchL has moved to the far side of another BChl-synthesis gene, $b c h H$, in the same operon, leaving $b c h N$ and $b c h B$ together (fig 4.2c). In at least two chloroplasts (Marchantia and Pinus), chlL and chlN are found together and in that order, while $c h l B$ has migrated elsewhere within the genome (fig 4.2d). In other chlorophyllous organisms, the available information is insufficient to derive any firm conclusions. 
a)

b)

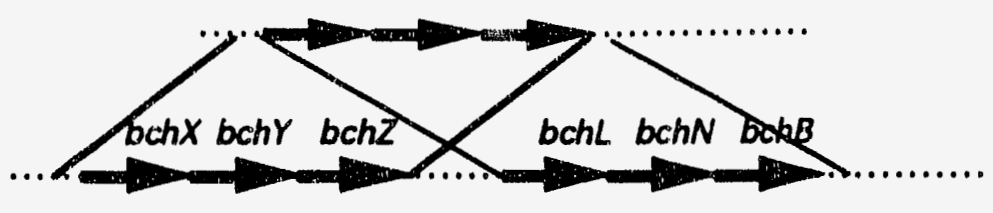

c)

$b c h X b c h Y b c h Z$

bchN bchB bchH

bchL

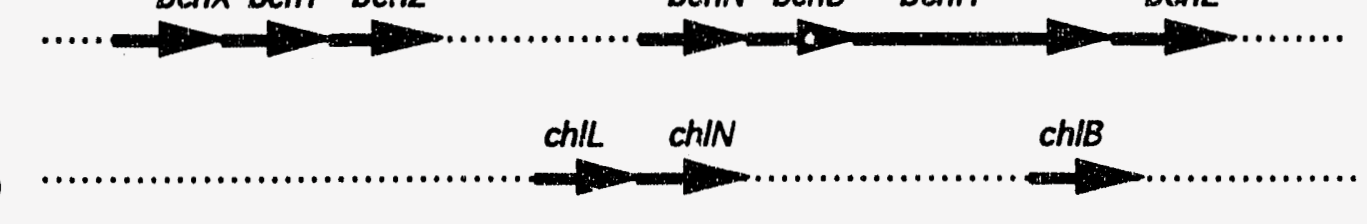

fig 4.2 Possible preservation of gene order among reductase

subunits. Genes are shown in bold arrows, other chromosomal DNA is shown by the dotted line. Orientation and distance between the various transcription units are not to scale. The gene duplication between (a) and (b) is shown by the slanted lines connecting the two. (a) The putative ancestral threesubunit reductase. (b) Duplicate copies are free to diverge and specialize. (c) Gene order in $R$. capsulatus. (d) Gene order in some cyanobacteria and some chloroplasts.

Organisms which currently possess only BChl, such as Rhodobacter, Heliobacter, and Chloroflexus might possess one or two reductase systems, depending on the timing of the gene duplication relative to the separation of the lineages. For instance, if the duplication took place subsequent to the divergence of the Chloroflexaceae (and is there was no lateral transfer of BChl-synthesis genes), then Chluroflexus may still possess a single enzyme system capable of performing both reactions. A bacterium with two reductase systems, on the other hand, has the potential to regulate the activities of each independently, allowing the simultaneous accumulation of both $\mathrm{Chl}$ and $\mathrm{BChl}$. 
This may be what has happened in the Chlorobiaceae, which use Bchla in the reaction centers and myriad distinct Chls in their antennae (Scheer 1991). Cyanobacteria do not make BChl, and they can be presumed to have lost the bchXYZ chlorin reductase.

The central theme of the "recapitulation theory" and the Granick Hypothesis is that biosynthetic pathways are extended one evolutionary step at a time. As soon as a plausible mechanism is proposed by which a particular pathway covld leap ahead two or more biochemical steps in a single evolutionary step, then both the recapitulation theory and the Granick Hypothesis can be called into question for that specific case. Such a mechanism is apparent in the common ancestry of the PChlide and chlorin reductases: The first PChlide-reducing $\mathrm{Chl}$ Fe protein system may have reduced both rings $B$ and $D$, thereby reducing the pheoporphyrin directly to a bacteriochlorin with a single enzyme system. Invention of this reductase would then have advanced photosynthetic reaction center pigments from a pheoporphyrin (see below) to a bacteriochlorin in a single evolutionary step. Only later, when a predecessor to modern cyanobacteria learned to halt the reaction after a single reduction, did $\mathrm{Chl}$ make its debut.

A key attraction of this model is that it resolves the apparent conflict between the Granick Hypothesis and the observed phylogenetic distribution of pigments used in photosynthetic reaction centers. There are four known tetrapyrrole reaction center pigments: Bchla, Bchlb, and Bchlg, and Chla. The bacteriochlorins are widely distributed among four of the five photosynthetic bacterial phyla (fig 4.3). Bchla is in the reaction center and antennae of the green, gliding bacteria (Chloroflexaceae) and most purple bacteria 


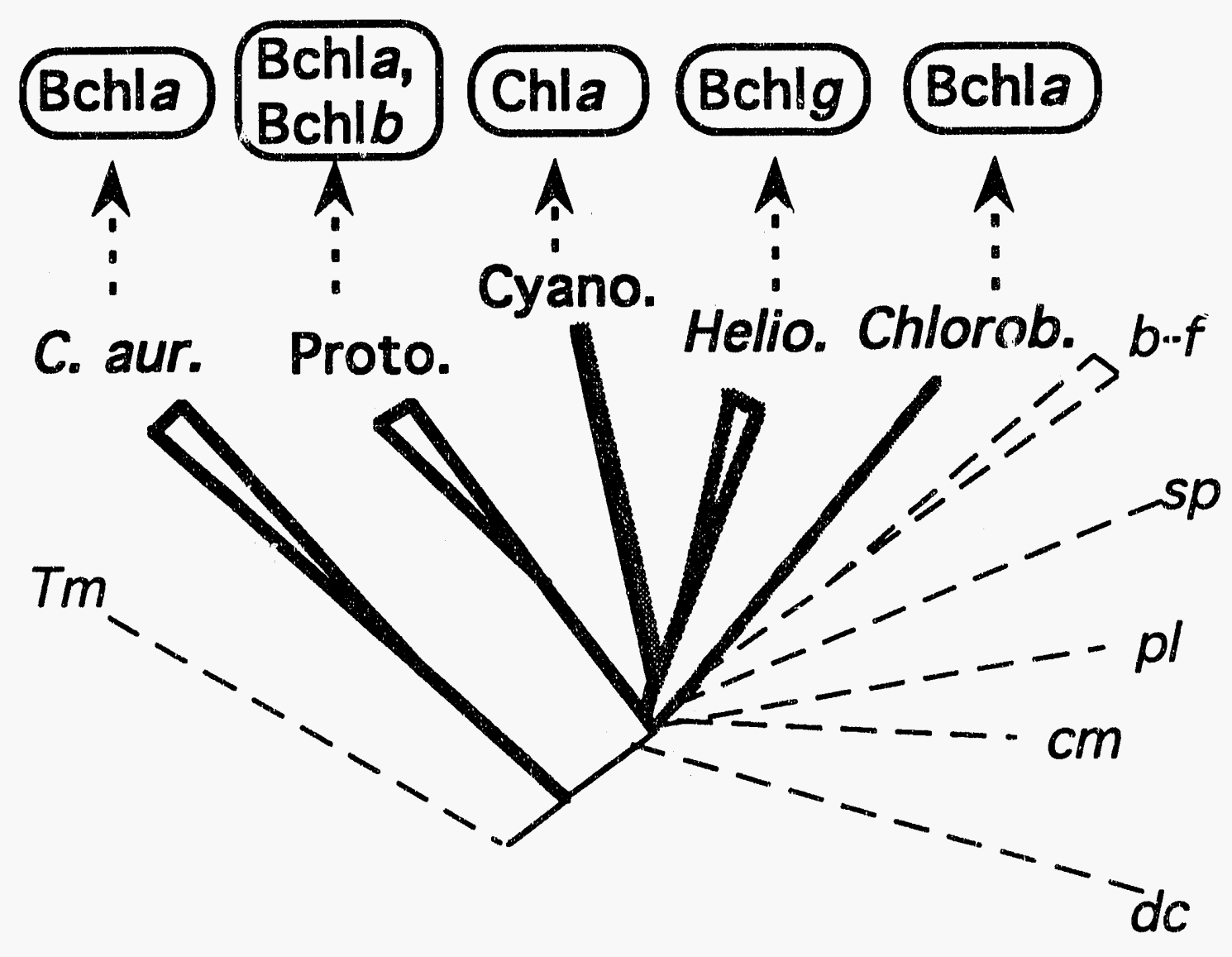

Fig 4.3. Phylogenetic distributions of reaction center

chromophores and reaction centers within the eubacteria. Lineages with PS-II (Quinone) type reaction centers are shown in dark solid lines, those with PS-I (FeS) type reaction centers are shown in shaded lines. Nonphotosynthetic bacterial lineages are shown with dashed lines. Structures of the chromophores are given in fig 4.4. Bacterial divisions represented are as follows: Tm, Thermatoga maratima; C. aur., Chloroflexus aurantiacus; Proto., proteobacteria; cyano, cyanobacteria; Helio, Heliobacterium chlorum; Chlorob, Chlorobium tepidum; b-f, bacteriods and relatives; sp, spirochetes; pl, planctomyces; $\mathrm{cm}$, chlamydiae; dc, deinococci and relatives. 


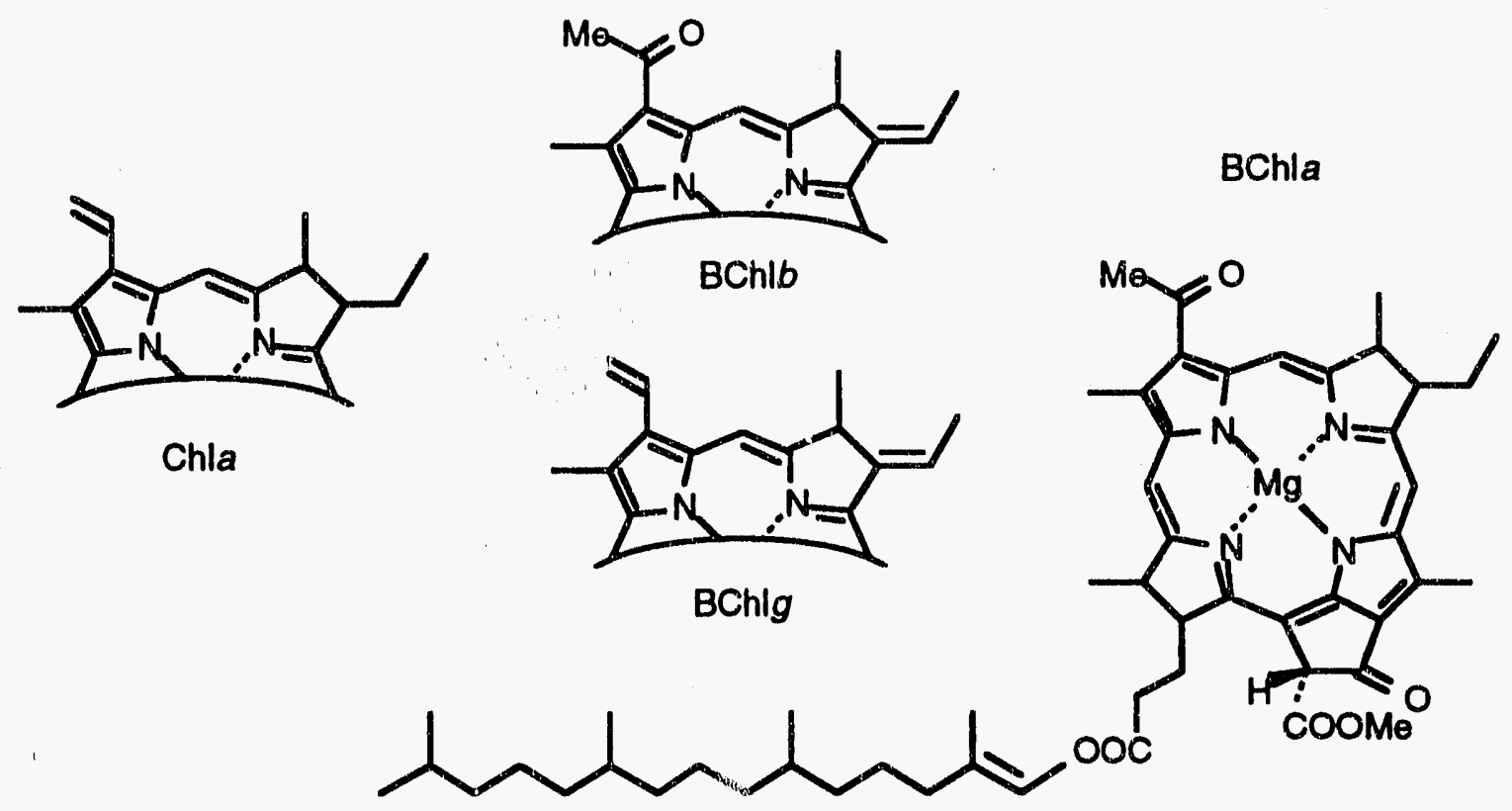

fig 4.4 Structures of the Reaction Center Chromophores. Structural elements in Chla, BChlb, and $\mathrm{BChl} g$ shared with $\mathrm{BChla}$ are not shown.

(Proteobacteria), including Rhodobacter capsulatus. Bchla is also the reaction center pigment of the green, sulfur bacteria (Chlorobiaceae). The 4-ethyl group of Bchla is replaced by an ethylidene in Bchlg in Heliobacteriacea within the gram positive lineage and in Bchlb in the Proteobacterium Rhodopseudomonas viridis (fig 4.4). The only group which uses Chla in place of a BChl in its photosynthetic reaction centers are the cyanobacterial chloroplasts. This group is photosynthetically the most sophisticated. Most cyanobacteria, but not all, couple two distinct photosystems to oxidize water, resulting in oxygen evolution. The broad phylogenetic distribution of $\mathrm{BChl}$ as the primary photoreceptor suggests that it may have become established 
among photosynthetic bacteria before the introduction of $\mathrm{Chl}$ into one specific lineage (cyanobacteria/chloroplasts).

Variable numbers of repetitive actions by a single enzyme in different biosynthetic contexts may be an important theme in the evolution of biosynthetic pathways. An example of this can be found among the biosynthetic enzymes for another important photosynthetic pigment, the carotenoids. Carotenoids are $\mathrm{C}_{40}$ polyenes that protect photosynthetic organisms against photooxidative damage, as well as participating directly in excitation transfer and other functions (Goodwin 1980, Trautman et al. 1990). All of the biological properties of carotenoids require that they contain extended systems of conjugated double bonds. Carotenoids are derived from phytoene, which contains nine double bonds arranged such that no more than three are conjugated together. Four successive dehydrogenations by the enzyme phytoene dehydrogenase (or "desaturase") convert phytoene to lycopene (tomato red), which is then cyclized in Chloroflexus and in plants to produce carotene (carrot orange). Phytoene dehydrogenase (the crtI product) performs only three dehydrogenation reactions in Rhodobacter to produce neurosporene (Armstrong et al. 1989a), the immediate precursor to lycopene in plants. Neurosporene is thein hydroxylated before being dehydrogenated by the $\operatorname{crtD}$ product and methylated to produce spheroidene. The amino acid sequence similarities between $c r t I$ and $c r t D$ demonstrate that they are derived from a common ancestor (Armstrong, Alberti, and Hearst 1990a). The modern crtl product is even capable of substituting for $\operatorname{crt} D$ when expressed at high levels (Bartley and Scolnik 1989). Although there is no way of knowing whether the common ancestor of crtD and crtl performed three 
dehydrogenation reactions or four, their situation is remarkably similar to that of the reductases in the (B)Chl synthesis pathways.

Quantitative Phylogenetic Analysis of the Iron Protein Subunits.

In order to test this hypothesis and to further examine the relationships among the three classes of $\mathrm{Fe}$ proteins, the amino acid sequences of eleven eubacterial und three archaebacterial nifH genes were aligned with those of four $\mathrm{Chl} \mathrm{Fe} \mathrm{proteins} \mathrm{(see} \mathrm{table} \mathrm{4.2).} \mathrm{A} \mathrm{computer-generated} \mathrm{global} \mathrm{tree}$ containing all sequences was built in which the Chl Fe proteins grouped together to the exclusion of all nifH proteins, and in which the root of the Chl Fe protein tree was on the $b c h X$ line (fig 4.5). (The analyses in this section were done in collaboration with Arend Sidow in the laboratory of Prof. Allan Wilson at U. C. Berkeley.) In order to test the statistical rigor of this tree, one could calculate the probability of every possible topology and compare that with the best one found by this method. However, with 18 sequences, there are $1.21 \times 10^{19}$ possible topologies relating them, such that a completely rigorous analysis is not possible. Fortunately, with a few simplifying assumptions, the tree can instead be built up from subsections, where each subsection can be rigorously examined. Phylogenetic trees relating the nifH,chlL-bchL, and $b c h X$ sequences were therefore built up by bootstrapping and random sampling with replacement analyses of several subsections of the data.

The four $\mathrm{Chl} \mathrm{Fe}$ proteins were considered first. The PChlide reductase Fe proteins (bchL-chlL) are assumed to be orthologous (related to each other by speciation events) while the chlorin reductase $\mathrm{Fe}$ protein $(b \operatorname{chX})$ is parologous to them (related by a gene duplication event). Of the three topologies that can 
Table 4.2

\begin{tabular}{|c|c|c|c|}
\hline abbreviatior & species & classification 9 & source $^{2}$ \\
\hline Rrub & Rhodospirillum rubrum & $\alpha-$ Protobacteria & M33774 \\
\hline Rcaps & Rhodobacter capsulatus & $\alpha-$ Protobacteria & X07866 \\
\hline Rmel & Rhizobium meliloti & $\alpha-$ Protobacteria & 101781 \\
\hline$T$ & Thiobacillus ferrooxidans & $\beta$-Protobacteria & M15238 \\
\hline K & Klebsiella preumoneae & rProtobacteria & $\mathrm{J} 01740$ \\
\hline$A z$ & Azotobacter vinelandii & $\gamma$-Protobacteria & M11579 \\
\hline An & Anabaena & cyanobacteria & V00001 \\
\hline $\boldsymbol{F}$ & Frankia sp. & High-GC Gram-positive & $M 21132$ \\
\hline $\operatorname{Cop} 1$ & Clostridium pasteurianum & low-GC Gram positive & $\times 07472$ \\
\hline $\mathrm{Cp} 2$ & Clostridium pasteurianum & low-GC Gram positive & $x 07473$ \\
\hline Cp3 3 & Clostridium pasteurianum & low-GC Gram positive & $\times 07474$ \\
\hline Mth1 & Methanococcus thermolithotrophicus & \multirow{2}{*}{\multicolumn{2}{|c|}{$\begin{array}{l}\text { Methanocciccales (Archia) } \times 07500 \\
\text { Methanocciccales (Archae) } \times 13830\end{array}$}} \\
\hline Mth3 3 A & Methanococcus thermolithotrophicus & & \\
\hline Mc vol & Methanococcus voltae & \multicolumn{2}{|c|}{ Methanocciccales (Archae) $\times 03777$} \\
\hline Miv & Methanobacterium ivanovii & Methanobacteriales ( " & ) $\times 07501$ \\
\hline$b \operatorname{chx} x^{4}$ & Rhodobacter capsulatus & $\alpha-$ Protobacteria & 211165 \\
\hline bochl. 5 R & Rhodobacter capsulatus & $\alpha$-Protobacteria & 211165 \\
\hline Plect chil. ${ }^{5} p$ & Plectonerna boryanum & cyanobacterium & note 2 \\
\hline Marc chil ${ }^{5} A$ & Marchantia polymorpha chloroplast & non-vascullar plant & $x 04465$ \\
\hline
\end{tabular}

Fe protein sequences used in phylogenetic analyses.

1 classification of bacteria is according to Woese (11987),

2 all nucleotide sequences are from GenBank, except fol $b c h X$ and $b c h L$, which are deposited in EMBL, and Plect chll, which is from ref (Fujita $e:$ al. 1992).

3 The two sequences are from Type III (Fe-dependent nitrogenase) associated $\mathrm{Fe}$ protein. The other nifH sequences are Type-I (Mo-Fedependent nitrogenase) associated Fe proteins.

4 Chlorin reductase $\mathrm{Fe}$ protein

5 PChlide reductase $\mathrm{Fe}$ protein 


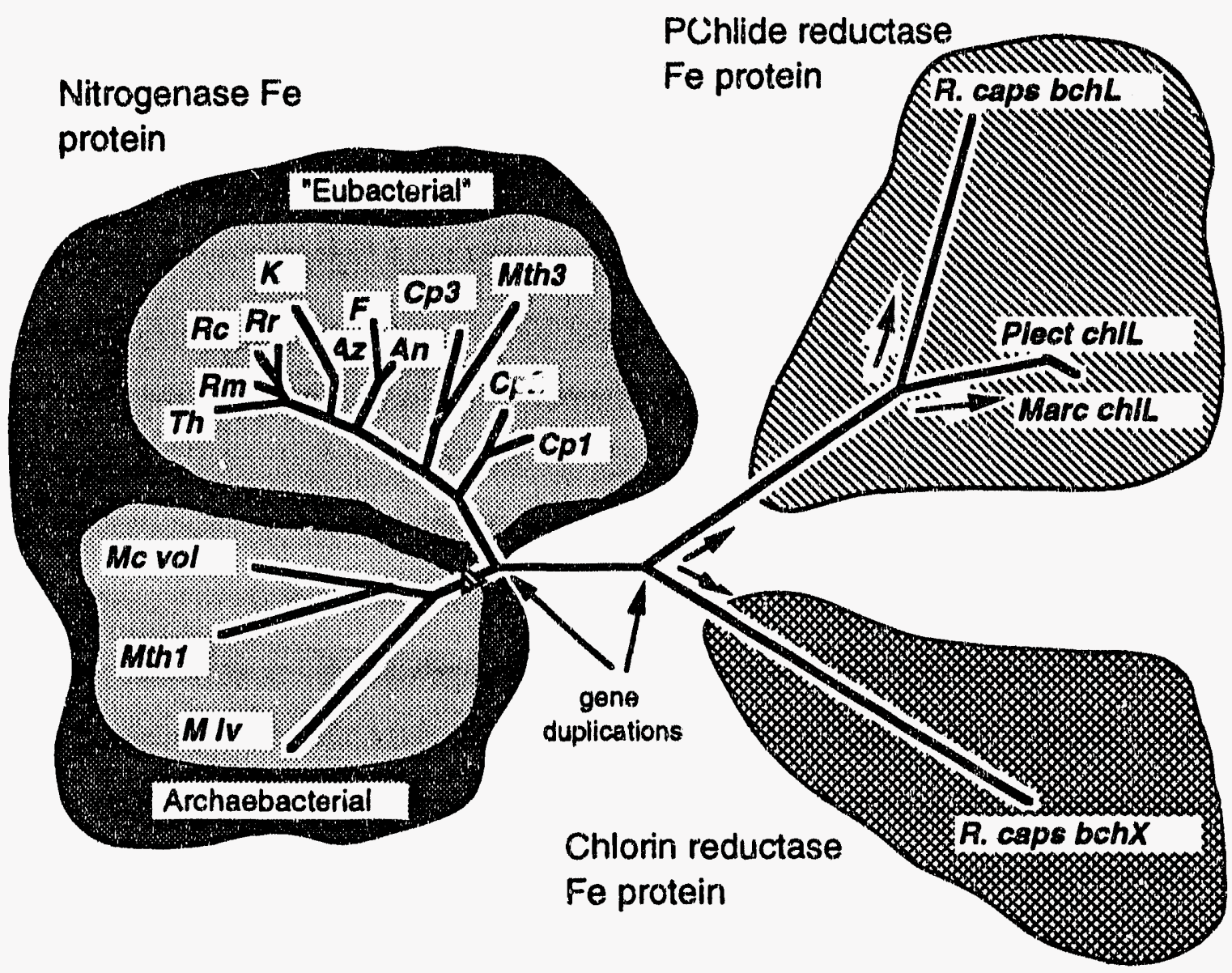

Fig 4.5 Unrooted global phylogenetic tree relating the nitrogenase, PChlide reductase, and chlorin reductase $\mathrm{Fe}$ proteins, based on the second positions of the 171 codons where homology is most securt. Branch lengths are proportional to the number of substitutions along each bra.lch. Assuming that the root lies within the nifH tree, the arrows represent the direction of evolutionary change, and the two nodes in the middle are gene duplication events, as indicated. Eubacterial nifH is indicated in quotes because of the grouping of an archaebacterial type III nifH within the same cluster. 


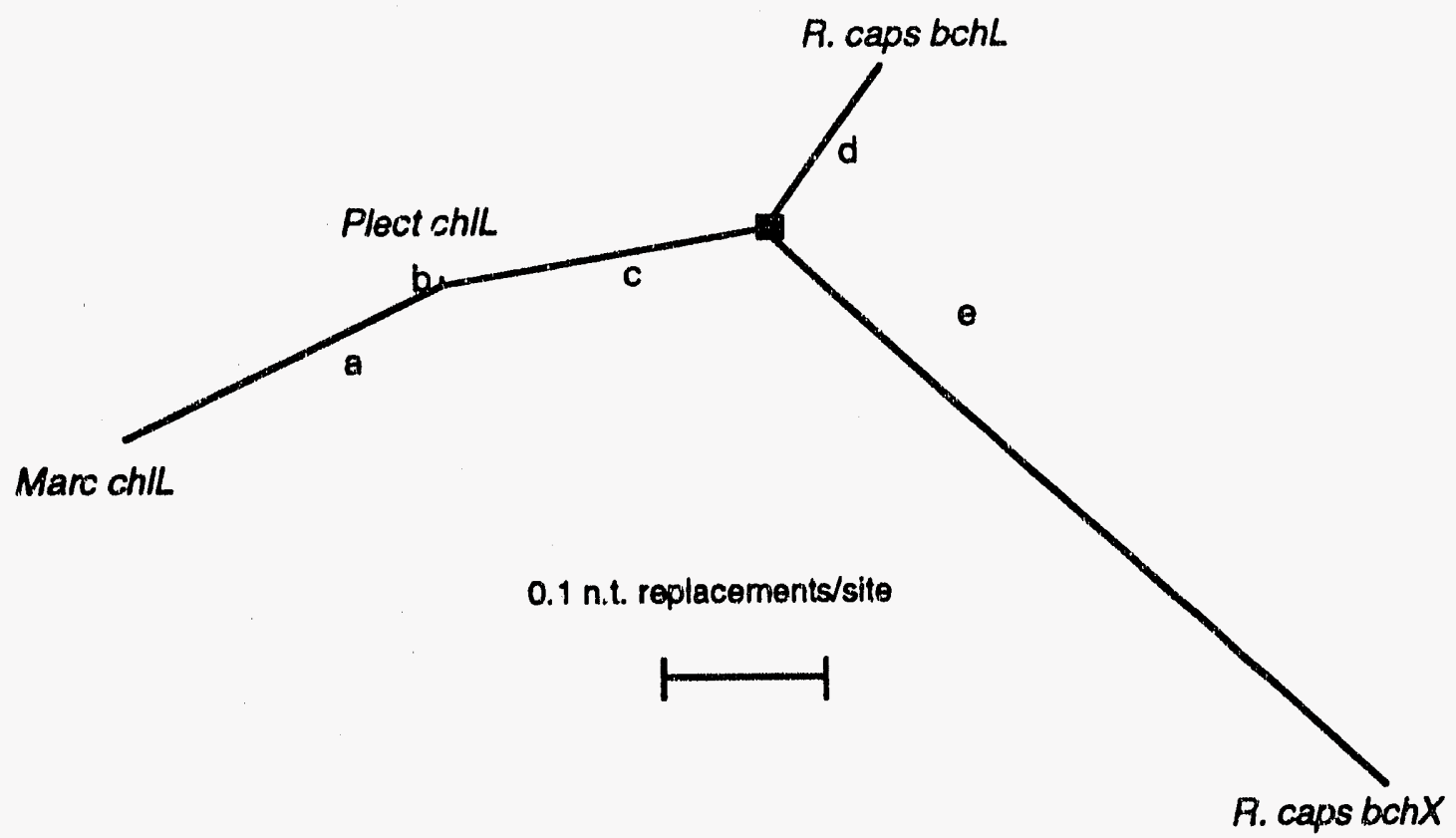

fig 4.6 Unrooted tree relating the $\mathrm{Chl}$ Fe proteins. Line segment lengths are proportional to the relative evolutionary distances, as shown by the scale bar. Calculated from first and second positions of 200 codons where homology is most secure among the Chl Fe proteins. The five line segments which were tested for attachment of the nitrogenase Fe protein tres are shown as letter a-e, while the significance of the black box is discussed in the text.

can be constructed to relate them, one was statistically significantly better than the others (fig 4.6).

Assuming that the $\mathrm{Chl} \mathrm{Fe}$ proteins arose from a nitrogenase $\mathrm{Fe}$ protein (see next section), placement of a root onto this tree give directionality to each of the line segments, because that point on the tree would be the farthest back 
in time, with the rest of the tree flowing from that point. For instance, if the root is along branch "e," this would imply that the black box shown in fig 4.6 is the speciation event separating purple bacteria from cyanobacteria and chloroplasts. If the root lies along any other branch, however, then the black box represents the gene duplication event which separated PChlide reductase Fe protein from chlorin reductase Fe protein. Since each rooting holds fundamentally distinct consequences for the history of photosynthetic organisms, a similar analysis was undertaken of the nitrogenase $\mathrm{Fe}$ proteins.

The first issue we considered in determining the relationship among the nitrogenase $\mathrm{Fe}$ proteins was the relationship between eubacterial and archaebacterial Type I and Type III enzymes. (Type I nitrogenases use a Mo-Fe cofactor, while Type III nitrogenases use Fe only. Each type of nitrogenase is usually associated with its own reductase, or Fe protein.) Because of the large number of nifH sequences available for analysis, these data were further subdivided. Six Type I proteins were considered in three groups, (Cp1 and Cp2), (Mth1 and Mvol), and (Rcaps1 and Rrub1), along with two Type III proteins, Mth3 and $\mathrm{Cp} 3$, whose relationship to each other and to the Type I proteins was unspecified. With five groups to be considered, there are fifteen possible topologies relating them. (There would have been $1.23 \times 10^{5}$ topologies to compare if all eight sequences were considered independently.) The two best of these are statistically indistinguishable, and are shown in fig 4.7. We find, as have others (Normand and Bousque, 1989, Normand et al. 1992), that the Type III nitrogenases form a separate cluster of their own, in spite of the presence of both types in both Eubacteria and Archaebacteria. 

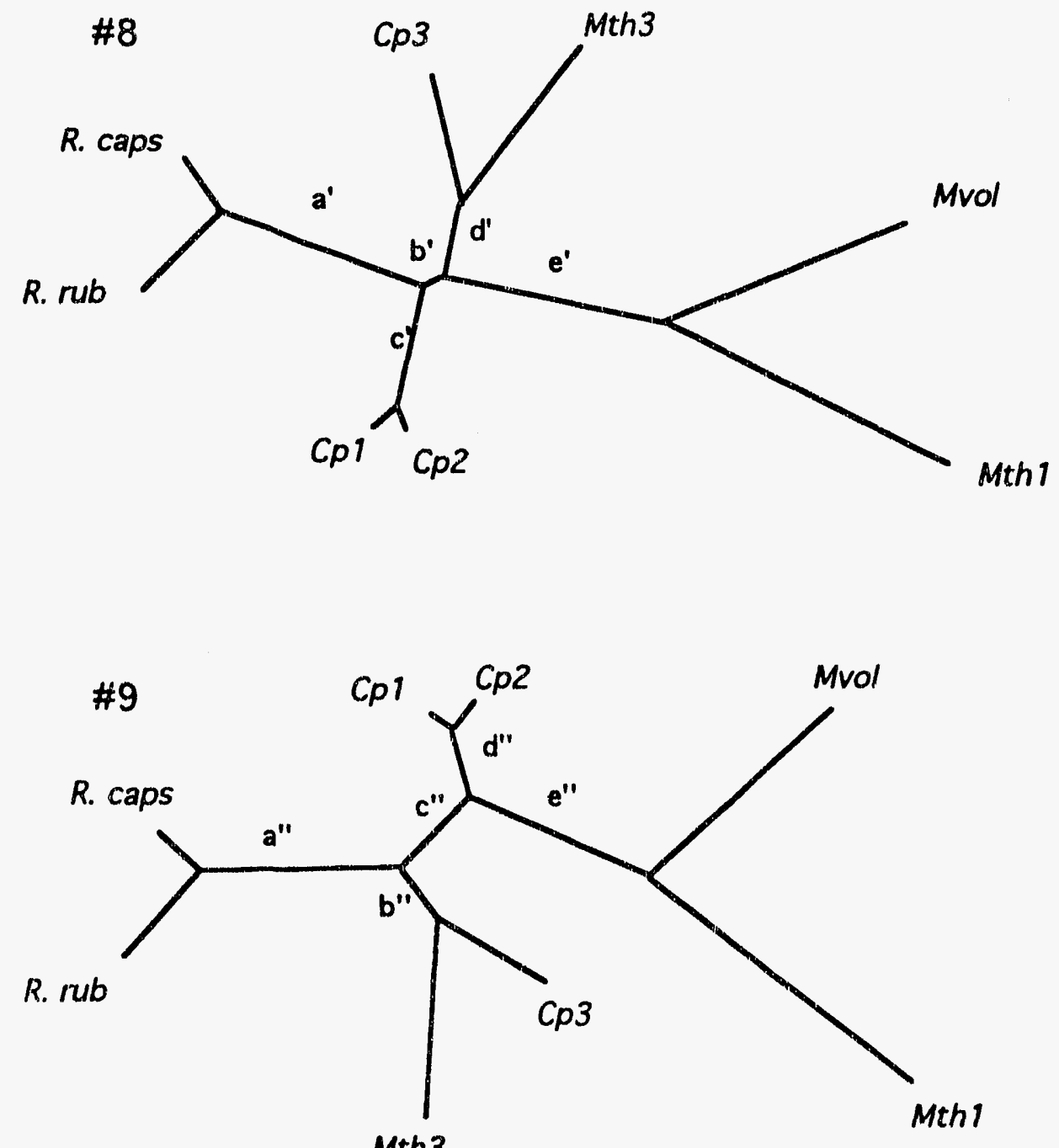

Mth3

\section{1 n.t. replacements/site}

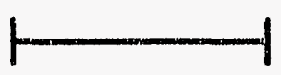

Fig 4.7 Unrooted tree relating Type I and Type III nitrogenase Fe proteins. Of the fifteen possible topologies relating the five groups, five could not be excluded on statistical grounds. Three of these are biologically nonsensical (They require that either a Type I protein converted into a type III and then back into a Type I, or that on two separate occasions a Type III converted into a Type I.) leaving the two trees shown here. Line segment lengths are proportional to the relative evolutionary distances between sequences, based on 252 codons. 
We are now in position to combine the two trees in a rigorous fashion, considering only the "reasonable" connections between them without having to consider all $1.25 \times 10^{9}$ topologies represented by the 12 sequences in the data set. Bootstrap analysis was performed on fifty separate topologies, in which each of the five line segments $(\mathrm{a}-\mathrm{e})$ of the $\mathrm{Chl} \mathrm{Fe}$ protein tree were connected one at a time to each of the five segments $\left(a^{\prime}-e^{\prime}\right.$ and $\left.a^{\prime \prime}-e^{\prime \prime}\right)$ in each of the two nifH trees $(5 \times 5 \times 2=50)$. In all statistically admissible topologies, the root of the ChI Fe protein tree is on the bchX lineage (fig 4.8). It is as yet

Marc chll

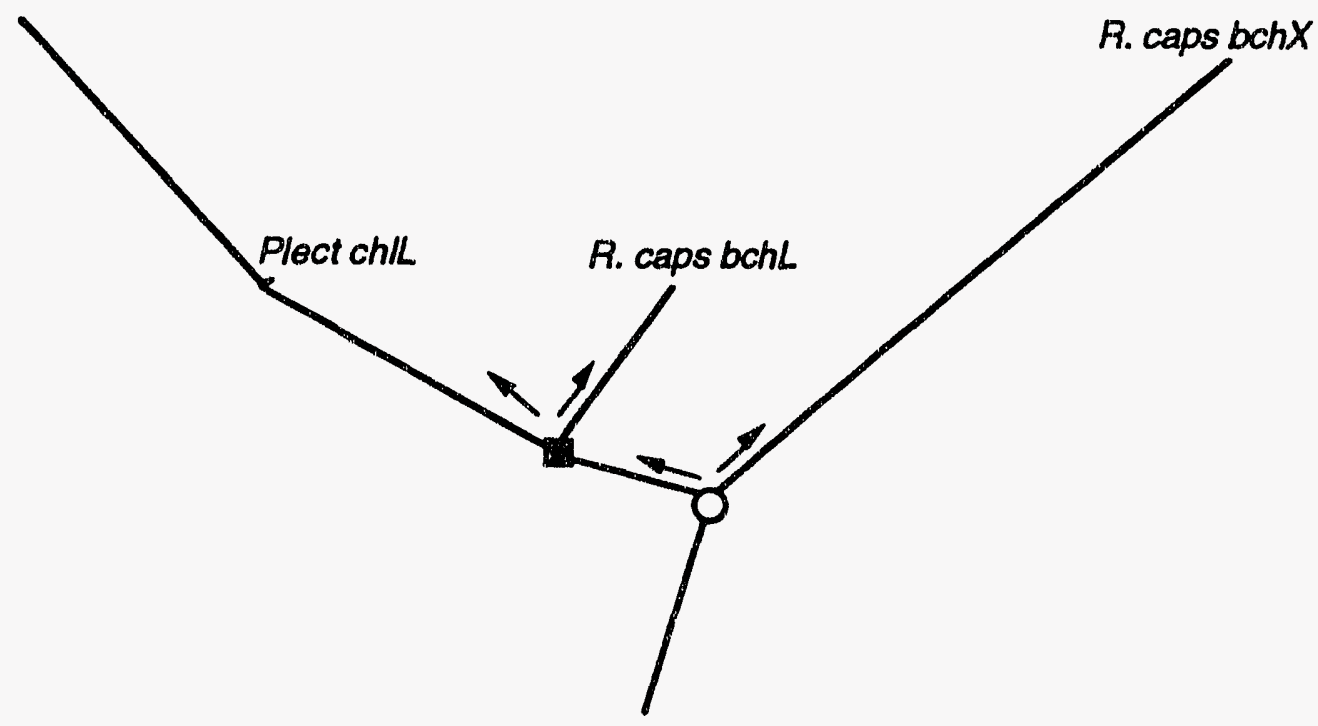

to nifH's

Fig 4.8 The root of the Chl Fe protein tree is on the $b c h X$ line. Evolutionary distances were calculated using the first and second positions of the 171 codons where homology among all proteins in the analysis is most secure. 
impossible to uniquely place that root onto a specific line segment of one of the two nitrogenase Fe protein trees. All five placements onto either tree \#8 or \#9 are statistically indistinguishable, as long as the connection is through the chlorin reductase $\mathrm{Fe}$ protein.

This arrangement offers strong evidence for the antiquity of BChl. The speciation event that separates bchL from chlL (the black box in fig 4.8) does not occur until after the separation of PChlide reductase $\mathrm{Fe}$ protein from Chlorin reductase $\mathrm{Fe}$ protein by gene duplication (open circle in fig 4.8). There is no speciation between the establishment of two $\mathrm{Chl} F \mathrm{Fe}$ proteins within the same cell and the split between Proteobacteria (which produce BChl and contain $b c h L$ ) and cyanobacteria (which produce Chl and contain $c h(L)$. Therefore, the common ancestor of cyanobacteria and Proteobacteria contained both a PChlide reductase and a chlorin reductase, and can therefore be presumed to have made $\mathrm{BChl}$.

\section{Photosynthesis vs. Nitrogen fixation}


nifH--all share a common ancestor. The common ancestor of all three classes (i.e., the molecule at the true root of the phylogenetic tree relating all members of this superfarnily of proteins) most likely participated in nitrogen fixation rather than in the synthesis of photosynthetic pigments. Nitrogen fixation is present among both eubacteria and archaebacteria. Comparison of nifH gene sequences from each Domain shows deep branching among the archaebacterial sequences and between them and the eubacterial sequences (this work and refs (Normand and Bousquet 1989, Souillard et al. 1988)). Based i. these results, Souillard et al. have posited that no lateral transfer of $n$ if 
genes has taken place between the two Domains in recent geological time, and that nif function is indeed very ancient (Souillard et al. 1988). The simplest explanation is that the common ancestor of Eubacteria and Archaebacteria reduced atmospheric nitrogen. The grouping of Type III enzymes from both Domains into a cluster to the exclusion of Type I enzymes adds further support to this position. Barring lateral transfer between the Domains, the most likely scenario represented by this topology is that the bacterium that gave rise to both Archaea and Eubacteria contained both Type $I$ and Type III nitrogenase iron proteins, and that the Type III has been lost in several lineages since then. If the rooted universal tree of Iwabe et al. (1989) is to be taken literally, then this organism was also the last common ancestor between Eubacteria and Eukaryotes. In contrast to nitrogen fixation, (B)Chl-based photosynthesis is present only among eubacteria (and the eukaryotes that have incorporated eubacteria as organelles). Presumably it arose subsequent to the split between Eubacteria and Archaebacteria. Two formal possibilities that cannot be ruled out are that (B)Chl-based photosynthetic archaebacteria are alive now and have simply not been found, or that they used to exist and have since become extinct.

Evolution of reaction center pigments before (B)ChI. The Reaction centers. There has been much speculation on the nature of the earliest photosyr.thetic reaction centers (Blankenship 1991, Nitschke and Rutherford 1991, Olson and Pierson 1987). Two kinds of reaction centers are in use among extant photosynthetic Bacteria and the Eukaria that have incorporated them as organelles (Nitschke and Rutherford 1991, Olson and Pierson 1987). PSI-like reaction centers are used by cyanobacteria/ 
chloroplasts, by the green sulfur bacteria (Chlorobiaceae) and in a modified form by the Heliobacteria, which group phylogenetically with the Grampositive bacteria (reviewed in (Nitschke and Rutherford 1991)). These lineages are shown as thick, shaded lines in fig 4.3. Preliminary sequence analysis of PSI-type reaction center peptides from Heliobacterium chlorum and from Chlorebium tepidum show that both are homologous with PSI reaction center peptides from cyanobacteria and plants (Trost, Brune, and Blankenship 1992). PSII-like ("Q-type") reaction centers are used by the green, gliding bacteria (typified by Chloroflexus), the purple bacteria (Proteobacteria) such as Rhodobacter, and cyanobacteria/chloroplasts. These lineages are shown as thick, solid lines in fig 4.3 .

All extant PSII-type reaction centers share sufficient sequence and structural similarities that they appear to be derived from a common ancestor (Beanland 1990, Blankenship 1991). When their core peptides are aligned and arranged into a phylogenetic tree (fig 4.9), the branching order of the different lineages has two surprising features: First, the reaction center $L$ and M components from $C$. aurantiacus group with those from the purple bacteria to the exclusion of the D1 and D2 peptides of cyanobacteria/chloroplasts, in spite of the fact that Chloroflexus branches off far more deeply in the $16 \mathrm{~S}$ rRNA-derived tree than the branch point between the cyanobacteria and the purple bacteria (Woese 1987). This may imply that a lateral transfer of photosynthetic functions has taken place between the purple bacteria and the Chloroflexaceae, as has been suggested (Beanland 1990, Blankenship 1991).

The second surprise is that there appear to have been independent gene duplication events which led to the differentiation of the heterodimeric reaction centers on both the cyanobacteria/chloroplast side of the tree and 
Photosynthetic Reaction Center Evolutionary Tree

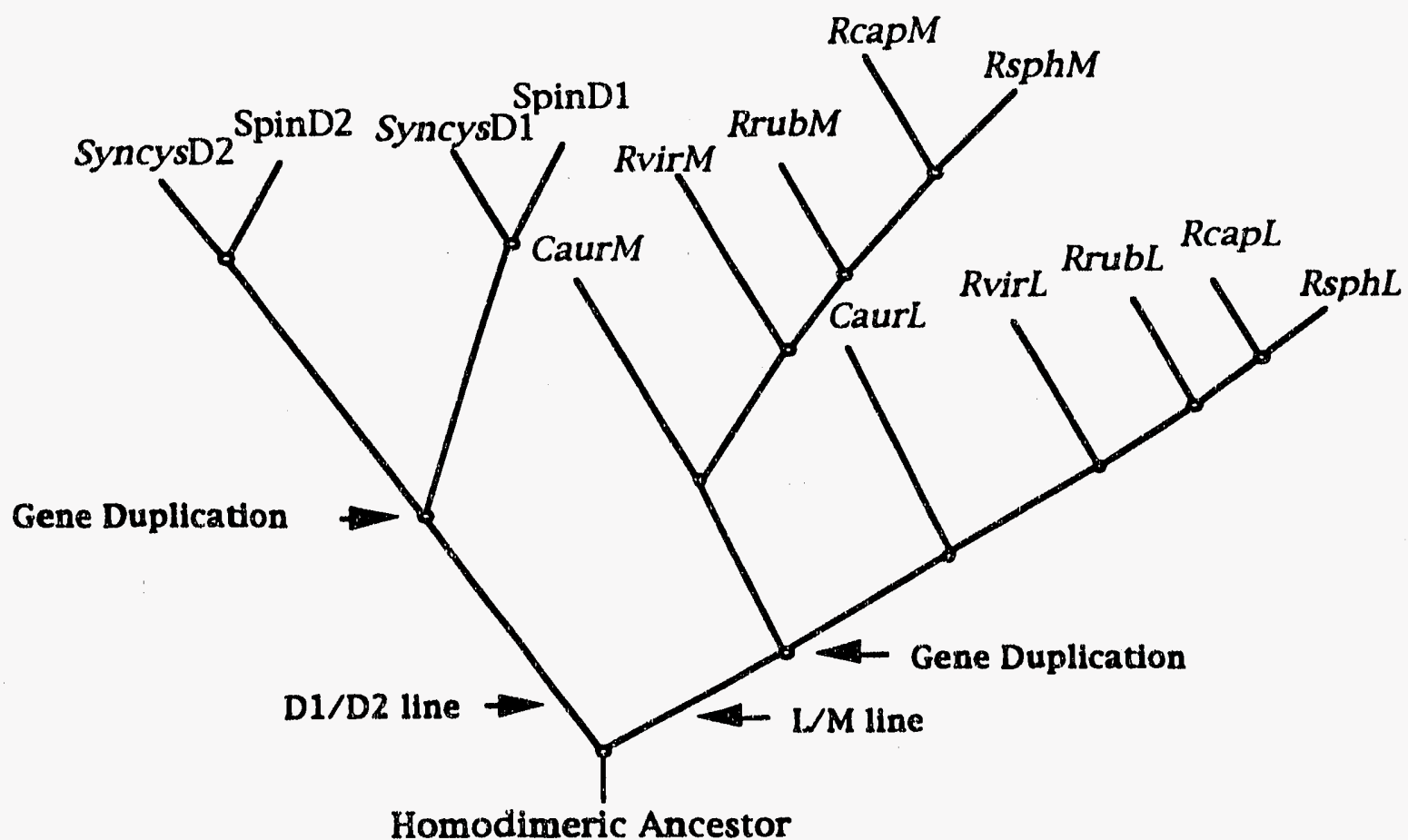

Fig 4.9 Evolutionary relationships within the photosynthetic reaction centers. From ref (Blankenship 1991). Abbreviations: Syncys, Synechocystis PCC6803; Spin, spinach chloroplast; Caur, Chloroflexus aurantiacus; Rvir, Rhodopseudomonas viridis; Rrub, Rhodospirillum rubrum; Rcap, Rhodobacter capsulatus; Rshp, Rhodobacter sphaeroides; D2, D1, L, and M, reaction center proteins from the respective organisms. 
the Proteobacteria/Chloroflexus side. The similarities of the $L$ and $M$ subunits and of the D1 and D2 subunits support models in which the common ancestor of all extant PSII-type reaction centers was itself homodimeric. PSI-like and PSII-like reaction centers share numerous structural and charge transfer characteristics, leading several authors to speculate that they may be derived from a common ancestor (Blankenship 1991. Nitschke and Rutherford 1991, Olson and Pierson 1987, Van Gorkom 1987). There may even be some remaining amino acid sequence similarities (Robert and Moerne-Loccoz 1990), although the evidence for this is tenuous. Depending on the interpretations given the evidence presented in the above references, one (if there was a single common ancestor for both PSI-like and PSII-like RCs) or two (one for PSI-like and one for PSII-like RCs) ancestral photosynthetic reaction centers can be assumed to account for all modern forms.

The pigments. The last common ancestor(s) of modern reaction centers could not have utilized a photopigment that was radically different from either Chl or $\mathrm{BChl}$ (such as protoporphyrin IX). This would have required the modern (B)Chl pigments (i.e., either a chlorin or a bacteriochlorin) to have been invented independently in each reaction center lineage following their divergence. On the other hand, it is highly unlikely that photosynthesis began with (B)Chl. Both $\mathrm{Chl}$ and $\mathrm{BChl}$ are several enzymatic reactions away from any related compound used in a biosynthetic pathway required for nonphotosynthetic processes. It can safely be assumed that the photosynthetic bacterium which carried the last common ancestor of modern reaction centers possessed uroporphyrinogen III, which is the first tetrapyrrole compound in the tetrapyrrole biosynthetic pathways, and the branch point between the (B)Chl/Heme and siroheme/factor $\mathrm{F}_{430}$ /Vitamin $\mathrm{B}_{12}$ pathways (see fig 1.5 in 
chapter 1). As such it is made by all organisms in the modern world and is likely to have been present in the progenitor of all extant life forms. A possible role for Uroporphyrinogen III in the origin of photosynthesis has been presented elsewhere (Larkum 1991, Mercer-Smith and Mauzerall 1984). It can probably also be assumed that the bacterium housing the last common ancestor of modern reaction centers also contained protoporphyrin IX, the branch point in the syntheses of heme and (B)Chl, because of the ubiquitous role of heme-containing cytochromes in photosynthetic electron transport circuits. The case has also been made for metallated protoporphyrin IX as the first photoactive pigment (Mauzerall 1990, Olson and Pierson 1987). Nevertheless, it is unlikely that a non-photosynthetic bacterium containing uroporphyrinogen III, or even protoporphyrin IX, simultaneously invented the enzymatic machinery to convert either of these compounds all the way to (B)Chl, as well as the membrane protein machinery to support photosynthetic charge transfer. In $R$. capsulatus, the products of at least eight genes are required just to convert protoporphyrin IX to chlorophyllide (see fig 1.8 in chapter 1). The inescapable conclusion is that other pigments were used in photosynthetic reaction centers between the time of the invention of photosynthesis and the introduction of modern pigments.

The most probable candidate as the immediate predecessor to modern reaction center pigments is a pheoporphyrin such as protochlorophyll. Pheoporphyrins are intermediate between protoporphyrins and chlorins, in that they contain the fifth, exocyclic ring, but the redox state of the pheoporphyrin macrocycle is two electrous more oxidized than the chlorins (fig 4.1). The Chlc pigment family used by some modern-day algae are, in effect, protochlorophyllide (PChlide) modified from C-17 propionate to C-17 
acrylate, and they are not usually esterified with long-chain isoprenoid tails (Scheer 1991). Chlc is used as a lighi-tharvesting antenna molecule and not in the reaction center, but it demonstrates that a pheoporphyrin is capable of biologically useful excitation transfer, and could have served as an early reaction center pigment (Larkunn 1991). We refer to the type of photosynthesis carried out by this presumptive organism as pheoporphyrinbased photosynthesis; however "while the pheoporphyrin may have been used as the primary photopigment within the cell, it may have been an unintended side product in the production of earlier components that were themselves the true photopigments. Either way, once the pheoporphyrin and an Fe-proteinbased reductase system were present in the same cell, the way was cleared for modern (B)Chl pigments to be synthesized.

\section{Light-independent vs. light-deperdent PChlide reduction.}

The enzymes discussed to this point all function in the dark. However, a light-dependent PChlide reductase has been well-studied in angiosperms and many other chlorophyllous Eukarya (Griffiths 1991). Many of these organisms also contain the light-independent enzyme system. Dual pathways to PChlide reduction have been observed in most of the major groups of Chl(but not BChl-) containing organisms, including green algae, bryophytes, pteridophytes, and gymnosperms. The light-independent chlLNB system has been lost on more than one occasion. Not only is it missing from all angiosperms examined to date, but also from Gnetalles, the gymnosperm sister group to the angiosperms (D. Burke, L. Raubeson, D. Stein, J. Hearst, in prep), suggesting that it was lost prior to the origin of flowering plants. Until recently, there had been no information as to which system was used among the cyanobacteria. Within recent years, it has become apparent that at least 
some cyanobacteria possess both a light-independent (Peschek et al. 1989b), chlLNB (Burke, Alberti, and Hearst 1991, Fujita et al. 1992) PChlide reductase system and a light-dependent pathway to Chl synthesis (Fujita et al. 1992). Is there any way of knowing which pathway is older? In this section I examine the evidence (none of which is definitive) as to which pathway to PChlide reduction is more ancient.

The first point that must be kept in mind is that the two systems are completely unrelated; i.e., neither is likely to have been derived directly from the other. The light-dependent enzyme is coded by a single gene which is active in lysates from $E$. coli cells infected with $\lambda \mathrm{gt}_{11}$ phage carrying the barley gene for PChlide reductase (Schultz et al. 1989). The action spectrum of this enzyme parallels the absorbance spectrum of PChlide, demonstrating that absorbance of a photon by the substrate activates the reaction (Griffiths 1991). This is in marked contrast to the subunit structure and proposed mechanism of the light-independent, Chl Fe protein-based reductions (above and ref (Burke, Alberti, and Hearst 1992a)). None of the subunits of PChlide or chlorin reductase bares any amino acid sequence similarity to the light-dependent PChlide reductase. Given such disparities between the two systems, they can be presunned to have had separate origins.

What lictle is known of the phylogenetic distribution of both PChlide reductase systems suggests that the light-dependent enzyme may be restricted to the cyanobacterial/chloroplast lineage. No other pathways to PChlide reduction are present in $R$. capsulatus in addition to the bchLNB system. Mutations in any of $b c h L, b c h N$, or $b \operatorname{chB}$ block accumulation of $B C h l$ whether the cultures are incubated in the light or in the dark (D. Burke, unpublished 
observation). This is further demonstrated by the inability of $b c h B$ and $b c h L$ mutants to grow photosynthetically (Yen and Marrs 1976, Zsebo and E. 1984a). Sirnilar PS- phenotypes have been reported for bchB and bchL mutants of $R$. sphaeroides (Coomber et al. 1990) These $\alpha$-Proteobacteria have either lost the light-dependent PChlide reductase or they never had it in the first place. Clearly it will be of interest to survey the other three photosynthetic bacterial divisions systematically for their choices of PClalide reductases. Based on the observaiton that the chlLNB-bchLNB system is present in both cyanobacteria and Proteobacteria, while the light-dependent system appears to be limited to only one of these two kingdoms, it is more likely that the first PChlide reductase contained a $\mathrm{Chl}$ Fe protein, and that the light-dependent enzyme is a more recent invention.

\section{Su m mary}

Three new lines of evidence have beerl put forth here regarding the antiquity of BChl. First, the overwhelming similarities batween PChlide reductase and chlorin reductase firmly establish that they share a common ancestor. The ancestral reductase may have performed one or both reductions, thereby circumventing the philosophical quandary presented by the recapitulation theory. Second, four out of the five photosynthetic bacterial divisions use a bacteriochlorophyll in their reaction centers, while only one (cyanobacteria and relatives) uses chlorophyll. Finally, our ability to place the root of the $\mathrm{Chl} F \mathrm{Fe}$ protein tree on the chlorin reductase lineage with confidence strongly suggests that both a PChlide reductase and a chlorin reductase were present in the bacterium that was the common ancestor between the proteobacteria and the cyanobacteria. 
Based on the evidence presented in the precedin: sections, I propose the following history of the nitrogenase/PChlide reductase/chlorin reductase Fe protein family: The ancestral Fe protein contributed its electrons to a catalytic complex that reduced atmospheric nitrogen. This complex may have been either type 1 or type III, although the latter is often favored because of its relative simplicity. Both types appear to have become established prior to the speciation event that separated the Eubacteria from the Archaebacteria. Subsequent to this event, there was a duplication of the nifH gene in the Eubacterial line. One of the copies was modified sufficiently to enable it to interact with a Pheoporphyrin-binding protein, thereby forming the first PChlide reductase. This reductase probably reduced its substrate twice to form a bacteriochlorin. Subsequent duplication of each of the subunits of this complex allowed the two copies to specialize toward reduction of a specific substrate. Loss of the chlorin reductase signalled the introduction of Chl. Nitrogenase $\mathrm{Fe}$ protein may therefore be responsible for the presence in the modern world of reduced reaction center pigments and thereby for the fact that grass is green. 
References

Albright, L. M., Ronson, C. W., Nixon, B. T. \& Ausubel, F. M. Identification of a gene linked to Rhizobium meliloti ntrA whose product is homologous to a family of ATP-binding proteins. J. Bact. 171, 1932-1941 (1989).

Allen, J. P., Feher, G., Yeates, T. O., Komiya, H. \& Rees, D. C. Structure of the reaction center from Rhodobacter sphaeroides R-26: protein-cofactor (quinones and Fe2t) interactions. Proc. Natl. Acad. Sci. 85, 8487.91. (1988).

Altuvia, S., Kornitzer, D., Teff, D. \& Oppenheim, A. B. Alternative mRNA structures of the cIII gene of bacteriophage $\lambda$ determine the rate of its translation initiation. J. Mol. Biol. 210, 265-280 (1989).

Armstrong, G. A., Alberti, M. \& Hearst, J. E. Conserved enzymes mediate the early reactions of carotenoid biosynthesis in nonphotosynthetic and photosynthetic prokaryotes. Proc. Natl. Acad. Sci. 87, 9975-9979 (1990).

Armstrong, G. A., Alberti, M., Leach, F. \& Hearst, J. E. Nucieotide sequence, organization, and nature of the protein products of the carotenoid biosynthesis gene cluster of Rhodobacter capsulatus. Mol. Gert. Geret. 216, 254 268 (1989).

Armstrong, G. A., Cook, D. N., Ma, D., Alberti, M., Burke, D. H. \& Hearst, J. E. Differential regulation of bacterial carotenoid biosynthesis genes by oxygen tension and identification of an evolutionarily conserved gene required for bacteriochlorophyll accumulation. (submitted to J. Bacterioll.) (1992). 
Avissar, Y. J., Ormerod, J. G. \& Beale, S. I. Distribution of delta-aminolevulinic acid biosynthetic pathways among phototrophic bacterial groups. Arch. Microbiol. 151, 513-519 (1989).

Bartley, G. E. \& Scolnik, P. A. Carotenoid biosynthesis in photosynthetic bacteria. Genetic characterization of the Rhodobacter capsulatus CrtI protein. J. Biol. Chem. 264, 13109-13113 (1989).

Bauer, C. E. (1992), submitted.

Bauer, C. E., Buggy, J. J., Yang, Z. \& Marrs, B. The superoperonal organization of genes from pigment biosynthesis and reaction center proteins is a conserved feature in Rhodobacter capsulatus: Analysis of overlapping bchB and puhA transcripts. Mol. Gen. Genet. 228, $433-444$ (1991).

Beale, S. I. \& Weinstein, J. D. Biosynthesis of 5-aminolevulinic acid in phototrophic organisms. Chlorophylls 385-406 (1991).

Beanland, T. J. Evolutionary relationships between "Q-type" photosynthetic reaction centres: Hypothesis-testing using parsimony. J. Theor. Biol. 145, 535545 (1990).

Belanger, F. C. \& Rebeiz, C. A. Chloroplast biogenesis XXVII Detection of novel chlorophyll and chlorophyll precursors in higher plants. Biochem. Biophys. Res. Comm. 88, 365-372 (1979). 
Belanger, F. C. \& Rebeiz, C. A. Chloroplast biogenesis Detection of divinyl protochlorophyllide in higher plants. J. Biol. Chem. 265, 1266-1272 (1980).

Benner, S. A., Ellington, A. D. \& Tauer, A. Modern metabolisın as a palimpsest of the RNA world. Proc. Natl. Acad. Sci. 86, $7054-7058$ (1989).

Berard, J. \& Gingras, G. The puhA structural gene coding for the $H$ subunit of the Rhodospirillum rubrum photoreaction center. Biochemistry and Cell Biology 69, 122-131 (1991).

Biel, A. J. \& Marrs, B. L. Transcriptional regulation of several genes for bacteriochlorophyll biosynthesis in Rhodopseudomonas capsulata in response to oxygen. J. Bact. $156,686-694$ (1983).

Blankenship, R. Origin and early evolution of photosynthesis. submitted for publication (1991).

Bogdanovi'c, M. Chlorophyll formation in the dark I. Chlorophyll in pine seedlings. Physiol. Plant. 29, 17-18 (1973).

Bowie, J. U., Luthy, R. \& Eisenberg, D. A method to identify protein sequences that fold into a known three-dimensional structure. Science 253, 164-170 (1991).

Brutlag, D. L., Dautricourt, J. P., Maulik, S. \& Relph, J. Improved sensitivity of biological sequence database searches. Computer Applications in the Biosciences 6, 237-245 (1990). 
Burke, D., Alberti, M. \& Hearst, J. Chlorophyll Fe proteins and other chlorophyll biosynthesis genes from Rhodobacter capsulatus to higher plants, abst T/pm-B5. Photochem. Photobiol. 53, Suppl., 85S-86S (1991).

Burke, D., Alberti, M. \& Hearst, J. Translational coupling in the bchCXYZ operon of Rhodobacter capsulatus and the generality of chlorophyll Fe proteins in chlorophyll and bacteriochlorophyll synthesis. in preparation (1992a).

Burke, D., Raubeson, L., Alberti, M., Hearst, J. \& Stein, D. Phylogenetic distribution of chloroplast and nuclear-encoded light-independent protochlorophyllide reductase. in preparation (1992d).

Burke, D. H., Alberti, M. \& Hearst, J. The bchFNBH bacteriochlorophyll synthesis genes of Rhodobacter capsulatus and identification of the third subunit of light-independent protochlorophyllide reductase in bacteria and plants. (1992b).

Choquet, Y., Rahire, M., Girard-Bascou, J., Erickson, J. \& Rochaix, J.-D. A chloroplast gene is required for the light-independent accumulation of chlorophyll in Chlamydomonas reinhardtii. EMBO J. 11, 1697-704 (1992).

Chu, G., Vollrath, D. \& Davis, R. Science 234, 1582- (1986).

Cohen-Bazire, G., Sistrom, W. R. \& Stanier, R. Y. Kinetic studies of pigment synthesis by non-sulfur purple bacteria. J. Cell. Comp. Physiol. 49, 25-68 (1957). 
Coleman, W. J. \& Youvan, D. C. Spectroscopic analysis of genetically modified photosynthetic reaction centers. Annual Review of Biophysics and Biophysical Chemistry 19, 333-367 (1990).

Coomber, S. A., Chaudhri, M., Connor, A., Britton, G. \& Hunter, C. N. Localised transposon Tn5 mutagenesis of the photosynthetic gene cluster of Rhodobacter sphaeroides. Molecular Microbiology 4, 977-989 (1990).

Das, A. \& Yanofsky, C. Restoration of a translational stop-start overlap reinstates translational coupling in a mutant $\operatorname{trp} B^{\prime}-\operatorname{trp} A$ gene pair of the Escherichia coli tryptophan operon. Nuc. Ac. Res. 17, 9333-9340 (1989).

de Smit, M. H. \& van Duin, J. Secondary structure of the ribosome binding site determines translational efficiency: A quantitative analysis. Proc. Natl. Acad. Sci. 87, 7668-7672 (1990).

Deisenhofer, J. \& Michel, H. Structures of bacterial photosynthetic reaction centers. Annual Review of Cell Biology 7, 1-23 (1991).

Deits, T. L. \& Howard, J. B. Effect of salts on Azotobacter vinelandii nitrogenase activities. Inihibition of iron chelation and substrate reduction. J. Biol. Chem. 265, 3859-3867 (1990).

Doolittle, R. F. Of URF's and ORF's a primer on how to analyze derived amino acid sequences. (1986). 
Drews, G. Structure and functional organization of light-harvesting complexes and photochemical reaction centers in membranes of phototrophic bacteria. Microbiol. Rev. 49, 59-70 (1985).

Ford, C., Mitchell, S. \& Wang, W.-y. Protochlorophyllide photoconversion mutants in Chlamydomonas reinhardtii. Mol. Gen. Genet. 184, 460-464 (1981).

Ford, C. \& Wang, W.-y. Three new yellow loci inChlamydomonas reinhardtii. Mol. Gen. Genet. 179, 259-263 (1980a).

Ford, C. \& Wang, W.-y. Temperature-sensitive yellow mutants of Chlamydomonas reinhardtii. Mol. Gen. Genet. 180, 5-10 (1980b).

Fujita, Y., Takahashi, Y., Chuganji, M. \& Matsubara, H. The nifH-like (frxC) gene is involved in the biosynthesis of chlorophyll in the filamentous cyanobacterium Plectonema boryanym. Plant Cell Physiol. 33, $81-92$ (1992).

Fujita, Y., Takahashi, Y., Kohchi, T., Ozeki, H., Ohyama, K. \& Matsubara, H. Identification of a novel nifH-like $(f r x C)$ protein in chloroplasts of the liverwort Marchantia polymorpha. Plant Mol. Biol. 13, 551-561 (1989).

Fujita, Y., Takahashi, Y., Shonai, F., Ogura, Y. \& Matsubara, H. Cloning, nucleotide sequence, and differential expression of the nifH andnifH-like $(f r x C)$ genes from the filamentous and nitrogen-fixing cyanobacterium Plectonema boryanym. Plant Cell Physiol. 32, 1093-1106 (1991). 
Gershoni, J. M., Shochat, S., Malkin, S. \& Ohad, I. Functional organization of the chlorophyll-containing complexes of Chlamydomonas reinhardi. Plant Physiol. 70, 637-644 (1982).

Gilbert, W. The RNA world. Nature 319, 618 (1986).

Goedheer, J. C. Visible absorption and fluorescence of chlorophyll and its aggregates in solution. The Chlorophylls 147-184 (1966).

Gogarten, J. P., Kibak, H., Dittrich, P., Taiz, L., Bowman, E. J., Bowman, M. J., Manolson, M. F., Poole, R. J., Date, T., Oshima, T., Jin, K., Denda, M. \& Toshda, M. Evolution of the vacuolar H+-ATPase: Implications for the origin of eukaryotes. Proc. Natl. Acad. Sci. 86, 6661-6665 (1989).

Goodwin, T. W. The Biochemistry of Carotenoids. 1, Plants (1980).

Gorchein, A. Magnesium protoporphyrin chelatase activity in Rhodopseudomonas spheroides. Biochem J. 127, 97-106 (1972).

Gorchein, A. Control of magnesium protoporphyrin chelatase activity in Rhodopseudomonas spheroides. Biochem J. 134, 833-845 (1973).

Granick, S. Evolution of haem and chlorophyll. Evolving Genes and Proteins $67-68$ (1965).

Gray, M. W. \& Doolittle, W. F. Has the endosymbiont hypothesis been proven? Microbiol. Rev. 46, $1-42$ (1982). 
Griffiths, W. T. Protochlorophyllide photoreduction. Chlorophylls 433-450 (1991).

Gussin, G. N. Three complementation groups in bacteriophage R17. J. Mol. Biol. 21, $435-453$ (1966).

Hartz, D., McPheeters, D. \& Gold, L. Influence of mRNA determinants on translation initiation in Escherichia coli. J. Mol. Biol. 218, 83-97 (1991).

Hausinger, R. P. \& Howard, J. B. Thiol reactivity of the nitrogenase Fe-protein from Azotobacter vinelandii. J. Biol. Chem. 258, 13486-13492 (1983).

Hayes, J. M., Kaplan, I. K. \& Wedeking, K. W. Precambrian organic geochemistry, preservation of the record. Earth's Earliest Biosphere 93-134 (1983).

Hearst, J. E., Alberti, M. \& Doolittle, R. F. A putative nitrogenase reductase gene found in the nucleotide sequence from the photosynthetic gene cluster of $R$. capsulata. Cell 40, 219-220 (1985).

Hearst, J. E. \& Sauer, K. Z Natuforsch 39c, 421-424 (1984).

Higgins, C. F., Hiles, I. D., Salmond, G. P. C., Gill, D. R., Downie, J. A., Evans, I. J., Holland, I. B., Gray, L., Buckel, S. D., Bell, A. W. \& Hermodson, M. A. A family of related ATP-binding subunits coupled to many distinct processes in bacteria. Nature $323,448-450$ (1986). 
Hinchigeri, S. B., Nelson, D. W. \& Richards, W. R. The purification and reaction mechanism of S-adenosyl-L-methionine:magnesium protoporphyrin methyltransferase from Rhodopseudomonas spheroides. Photosynthetica 18, 168-178 (1984).

Hiratsuka, J., Shimada, H., Whittier, R., Ishibashi, T., Sukamoto, M., Mori, M., Kondo, C., Honji, Y., Sun, C. R. \& Meng, B. Y. The complete sequence of the rice (Oryza sativa) chloroplast genome: Intermolecular recombination between distinct IRNA genes accounts for a major plastid DNA inversion during the evolution of the cereals. Mol. Gen. Genet. 217, 185-194 (1989).

Howard, J. B., Davis, R., Moldenhauer, B., Cash, V. L. \& Dean, D. Fe:S Cluster ligands are the only cysteines required for nitrogenase Fe-protein activity. $J$. Biol. Chem. 264, 11270-11274 (1989a).

Howard, J. B., Deits, T. L., Anderson, G. L., Maroney, M., Que, L. \& Hausinger, R. P. Nitrogen Fixation and $\mathrm{CO}_{2}$ Metabolism 153-162 (1985).

Hu, N. T. \& Marrs, B. L. Characterization of the plasmid DNAs of Rhodopseudomonas capsulata. Arch. Microbiol. 121, $61-69$ (1979).

Hunter, C., McGlynn, P., Ashby, M., Burgess, J. \& Olsen, J. DNA sequencing and complementation/deletion analysis of the bchA-puf operon region of Rhodobacter sphaeroides: in vivo mapping of the oxygen-regulated puf promoter. 5, 2649-2661 (1991). 
Ivey-Hoyle, M. \& Steege, D. A. Translation of phage f1 gene VII occurs from an inherantly defective initiation site made functional by coupling. J. Mol. Biol. 208, 233-244 (1989).

Iwabe, N., Kuma, K., Hasegawa, M., Osawa, S. \& Miyata, T. Evolutionary relationship of archaebacteria, eubacteria, and eukaryotes inferred from phylogenetic trees of duplicated genes. Proc. Natl. Acad. Sci. 86, 9355-9359 (1989).

Jaeger, J., Turner, D. \& Zuker, M. Improved predictions of secondary structures for RNA. Proc. Natl. Acad. Sci. 86, 7706-7710 (1989).

Joyce, G. F. The rise and fall of the RNA world. The New Biologist 3, 399-407 (1991).

Kiley, P. J. \& Kaplan, S. Molecular genetics of photosynthetic membrane biosynthesis in Rhodobacter sphaeroides. Microbiological Reviews 52, 50-69 (1988).

King, C. C. Did membrane electrochemistry precede translation? Origins of Life and Evolution of the Biosphere 20, 15-25 (1990).

Kohara, Y., Akiyama, K. \& Isono, K. The physical map of the whole E. coli chromosome: Application of a new strategy for rapid analysis and sorting of a large genomic library. Cell 50, $495-508$ (1987). 
Komiya, H., Yeates, T. O., Rees, D. C., Allen, J. P. \& Feher, G. Structure of the reaction center from Rhodobacter sphaeroides R-26 and 2.4.1: symmetry relations and sequence comparisons between different species. Proc. Natl. Acad. Sci. 85, 9012-9016. (1988).

Komiya, H. and Rees, D. (Submitted to Science) (1992).

Kyte, J. \& Doolittle, R. A simple method for displaying the hydropathic character of a protein. J. Mol. Biol. 157, 105-132 (1982).

Lake, J. A. Tracing origins with molecular sequences: metazoan and eukaryotic beginnings. Trends in Biochemical Sciences 16, $46-50$ (1991).

Larkum, A. W. D. Evolution of Chloropl ylls. Chlorophylls 367-384 (1991).

Lascelles, J. The regulation of heme and chlorophyll synthesis in bacteria. Ann. N. Y. Acad. Sci. 244, 334-347 (1975).

Lee, W. Y., Park, J. H., Kim, B. S., J., H. M. \& Hăhn, B. S. Chlorophyll derivatives (CpD) extracted from silk worm excreta are sperifically cytotoxic to tumor cells in vitro. Yonsei Medical Journal 31, 225-233 (1990).

Leeper, F. J. Intermediate steps in the biosynthesis of chlorophylls. Chlorophylls 407-432 (1991). 
Liritholm, J. \& Gustafsson, P. Homologs of the green algal gidA gene and the liverwort $f r x C$ gene are present on the chloroplast genomes of comifers. Plant Molecular Biology 17, 787-798 (1991).

Ma, D., Cook, D., O'Brien, D., and Hearst, J. E. Analysis of the promoter and regulatory sequences of an oxygen-regulated bch promoter in Rhodobacter capsulatus by site-directed mutagenesis. (Submitted to J. Bacteriol.) (1992).

Madigan, M. D. \& Guest, H. Growth of a photosynthetic bacterium anaerobically in darkness, supported by "oxidant-dependent" sugar fermentation. Arch. Microbiol. 117, 119-122 (1978).

Marre, B. Mobilization of the genes for photosynthesis from Rhodopseudomonas capsulata by a promiscuous plasmid. J. Bact. 146, 1003-1012 (1981).

Masinovsk'y, Z., Lazovaya, G. I., Sivash, A. A. \& Drasner, M. Porphyrinproteinoid complexes as models of prebiotic photosensitizers. BioSystems 22 , 305-310 (1989).

Matringe, M., Camadro, J.-M., Labbe, P. \& Scalla, R. Protoporphyrinogen oxidase as a molecular target for diphenyl ether herbicides. Biochem. J. 260, 231-235 (1989).

Mauzerall, D. The condensation of porphobilinogen to uroporphyrinogen. $J$. Am. Chem. Soc. 82, 2605 (1960). 
Mauzerall, D. Bacteriochlorophyll and photosynthetic evolution. The

Photosynthetic Bacteria. R. K. Clayton and W. R. Sistrom, eds. pp 223-231 (1978).

Mauzerall, D. C. The photochemical origins of life and photoreaction of ferrous ion in the archaean oceans. Origins of Life and Evolution of the Biosphere 20, 293-302 (1990).

McGlynn, P. \& Hunter, C. N. (Submitted to Mol. Microbiol.) (1992).

Mercer-Smith, J. A. \& Mauzerall, D. C. Photochemistry of porphyrins: A model for the origin of photosynthesis. Pholochemistry and photobiology 39, 397-405 (1984).

Meyer, J. The evolution of ferredoxins. Trends in Evol and Ecol. 3, 222-226 (1988).

Min Jou, W., Haegerman, G., Ysbaert, M. \& Fiers, W. Nucleotide sequence of the gene coding for the bacteriophage MS2 coall protein. Nature (London) 237, 8288 (1972).

Morowitz, H. J., Heinz, B. \& Deamer, D. W. The chemical logic of a minimal protocell. Origins of Life and Evolution of the Biosphere 18, 281-287 (1988).

Mortenson, L. E. \& Thornley, R. N. F. Stucture and function of nitrogenase. Ann. Rev. Biochem. 48, 387-418 (1979). 
Nitschke, W. \& Rutherford, A. W. Photosynthetic reaction centres: Variations on a common structural theme? TIBS 16, $241-245$ (1991).

Normand, P. \& Bousquet, J. Phylogeny of nitrogenase sequences in Frankia and other nitrogen-fixing microorganisms. J. Mol. Evol. 29, 436-447 (1989).

Normand, P., Gouy, M., Cournoyer, B. \& Simonet, P. Nucleotide sequence of nifD from Frankia alni strain Arl3: phylogenetic inferences. Molecular Biology and Evolution 9, 495-506 (1992).

Ogura, Y., Takemura, M., Yamato, K. \& others. Cloning and nucleotide sequence of a frxC-ORF469 gene cluster of Synechocystis PCC.6803--Conservation with liverwort chloroplastfrxC--orf465 and nif operon. Bioscience, biotechnology, and biochemistry 56, 788-793 (1992).

Ohyama, K., Fukuzawa, H., Kohchi, T., Shirai, H., Sano, T., Sano, S., Umesono, K., Shiki, T., Takeuchi, M., Chang, Z., Aota, S.-i., Inokuchi, H. \& Ozeki, H. Chloroplast gene organization deduced from complete sequence of liverwort Marchantia polymorpha chloroplast DNA. Nature (London) 322, 572-574 (1986).

Olson. J. M. \& Pierson, B. K. Evolution of reaction centers in photosynthetic prokaryotes. International Review of Cytology 108, 209-248 (1987).

Orme-Johnson, W. H. Molecular basis of biological nitrogen fixation. Ann. Rev. Biophys. Biophys. Chem. 14, 419-459 (1985). 
Ostrowski, J., Wu, J.-Y., Rueger, D. C., Miller, B. E., Siegel, L. \& Kredich, N. Characterization of the cysJIH regions of Salmonella typhimurium and Escherichia coli B. DNA sequences of cysI and $c y s H$ and a model for the siroheme-. $\mathrm{Fe}_{4} \mathrm{~S}_{4}$ active center of sulfite reductase hemoprotein based on amino acid homology with spinich nitrite reductase. J. Biol. Chem. 264, 15726-15737 (1989).

Paddock, M. L., McPherson, P. H., Feher, G. \& Okamura, M. Y. Pathway of proton transfer in bacterial reaction centers: Replacement of serine-L223 by alanine inhibits electron and proton transfers with reduction of quinone to dihydroquinone. Proc. Natl. Acad. Sci. 87, 6803-6807 (1990).

Paddock, M. L., Rongey, S. H., Feher, G. \& Okamura, M. Y. Pathway of proton transfer in bacterial reaction centers: Replacement of glutamic acid 212 in the $L$ subunit by glutamine inhibits quinone (secondary acceptor) turnover. Proc. Natl. Acad. Sci. 86, 6602-6606 (1989).

Park, J. H., Lee, W. Y., Hahn, B. S., J., H. M., Yang, W. I. \& Kim, B. S. Chlorophyll derivatives--a new photosensitizer for photodynamic therapy of cancer in mice. Yonsei Medical Journal 30, 212-218 (1989).

Peschek, G. A., Hinterstoisser, B., Pineau, B. \& Missbichler, A. Lightindependent NADPH-protochlorophyllide oxidoreductase activity in purified plasma membrane from the cyanobacterium Anacystis nidulans. Biochem \& Biophys Res Comm 162, 71-78 (1989a). 
Peschek, G. A., Hintertoisser, B., Wastyn, M., Kuntner, O., Pineau, B., Missbilcher, A. \& Lang, J. Chlorophyll precursors in the plasma membrane of a cyanobacterium Anacystis nidulans. Characterization by spectrophotometry, spectrofluorometry, solvent partition, and high performance liquid chromatography. J. Biol. Chem. 264, 11827-11832 (1989b).

Peschek, G. A., Villgrater, K. \& Wastyn, M. Respiratory protection of the nitrogenase in dinitrogen-fixing cyanobacteria. Plant and Soil 137, 17.24 (1991).

Pierson, B. K. \& Thornber, J. P. Isolation and spectral characterization of photochemical reaction centers from the thermophilic green bacterium Chloroflexus aurantiacus strain J-10-fl. Proc. Natl. Acad. Sci. 80, $80-84$ (1983).

Pudek, M. R. \& Richards, W. R. A possible alternate pathway of bacteriochlorophyll biosynthesis in a mutant of Rhodopseudomonas sphaeroides. Biochemistry 14, 3132-3137 (1975).

Richard, M. \& Bellemare, G. Nucleotide sequence of Chlamydomonas moewusii chloroplastic tRNAThr. Nuc. Ac. Res. 18, 2061 (1990).

Richards, W. R., Fidai, S., Gibson, L., Lauterbach, P., Snajdarova, I., Valera, V., Wieler, J. S. \& Yee, W. C. Enzymology of the magnesium branch of chlorophyll and bacteriochlorophyll biosynthesis. Photochemistry and Photobiology 53, 84S-85S (1991). 
Richards, W. R. \& Lascelles, J. The biosynthesis of bcteriochlorophyll. The characterization of latter stage intermediates from mutants of Rhodopseudomonas sphaeroides. Biochem. 8, 3473-3482 (1969).

Riley, M. \& Krawiec, S. Genome Organization. Escherichia coli and Salmonella typhimurium cellular and molecular biology 2, 967-981 (1987).

Rivera, M. C. \& Lake, J. A. Evidence that eukaryotes and eocyte prokaryotes are immediate relatives. Science $257,74-76$ (1992).

Robert, B. \& Moenne-Loccoz, P. Current Research in Photosynthesis 1, 65-68 (1990).

Roitgrund, C. Deletion mapping of two chloroplast genome functions in the green algaChlamydomonas reinhardtii. Ph. D. thesis. Chicago University (1989).

Roitgrund, C. \& Mets, L. J. Localization of two novel chloroplast genome functions: trans-splicing of RNA and protochlorophyllide reduction. Curr. Genet. 17, 147-153 (1990).

Sambrook, J., Fritsch, E. F. \& Maniatis, T. Molecular Cloning, A Laboratory Manual. (1989).

Sanger, F., Nicklen, S. \& Coulson, A. R. DNA sequencing with chain terminating inhibitors. Proc. Natl. Acad. Sci. 74; $5463-5467$ (1977). 
Scheer, H. Structure and occurrence of chlorophylls. Chlorophylls 3-30 (1991).

Schidlowski, M. A 3,800-million-year isotopic record of life from carbon in sedimentary rocks. Nature $333,313-318$ (1988).

Schidlowski, M., Hayes, J. M. \& Kaplan, I. R. Isotopic inferences of ancient biochemistries: Carbon, sulfur, hydrogen, and niliogen. Earth's Earliest Biosphere 149-186 (1983).

Schopf, J. W. Paleobiology of the Archaen. The Proterozoic Biosphere chapter 1 (1992).

Schopf, J. W. \& Packer, B. M. Early Archaen (3.3-billion to 3.5 billion-year old) microfossils from Warrawoona Group, Australia. Science 237, $70-73$ (1987).

Schultz, J. E. \& Weaver, P. F. Fermentation and anaerobic respiration by Rhodopsirillum rubrum and Rhodopseudomonas capsulata.J. Bact. 149, 181-190 (1982).

Schultz, R., Steinmüller, K., Klass, M., Forreiter, C., Rasmussen, S., Hiller, C. \& Apel, K. Nucleotide sequence of a cDNA coding for the NADPHprotochlorophyllide oxidoreductase (PCR) of barley (Hordeum vulgare L.) and its expression in Escherichia coli. Mol. Gen. Genet. 217, 355-361 (1989).

Sganga, M. W. \& Bauer, C. E. Regulatory factors controlling photosynthetic reaction center and light-harvesting gene expression in Rhodobacter capsulatus. Cell 68, 945-954 (1992). 
Shinozaki, K., Ohme, M., Tanaka, M., Wakasugi, T., Hayashida, N., Matsubayashi, T., Zaita, N., Chunwongse, J., Yamaguchi-Shinozaki, K., Ohto, C., Totazawa, K., Obokata, J., Meng, B. Y., Sugita, M., Deno, H., Kamogashira, T., Yamada, K., Kusuda, J., Takaiwa, F., Kato, A., Tohdoh, N., Shimada, H. \& Sugiura, M. The complete nucleotide sequence of the tobacco chloroplast genome: Its gene organization and expression. EMBO J. 5, 2043-2049 (1986).

Souillard, N., Magot, M., Possot, O. \& Sibold, L. Nucleotide sequence of regions homologous to nifH (nitrogenase Fe pretein) from the nitrogen-fixing archaebacteria Methanococcus thermolithotrophicus and Methanobacterium ivanovii: Evolutionary implications. J. Mol. Evol. 27, 65-76 (1988).

Stüber, D. \& Bujard, H. Transcription from efficient promoters can interfere with plasmid replication and diminish expression of plasmid specified genes. EMBO J 1, 1399-1404 (1982).

Studier, F., Rosenberg, A., Dunn, J. \& Dubendorff, J. Use of T7 RNA polymerase to direct expression of cloned genes. 185, 60-89 (1990).

Suwanto, A. \& Kaplan, S. Physical and genetic mapping of the Rhodobacter sphaeroides 2.4.1 genome: Genome size, fragment identification, and gene localization. J. Bacteriol. 171, 5840-5849 (1989a).

Suwanto, A. \& Kaplan, S. Physical and genetic mapping of the Rhodobacter sphaeroides 2.4 .1 genome: Presence of two unique circular chromosomes. $J$. Bacteriol. 171, $4850-4859$ (1989b). 
Suzuki, J. Y. \& Bauer, C. E. Light-independent chlorophyll biosynthesis: Involvement of the chloroplast gene, gidB (frxC). Plant Cell (in press, 1992).

Taylor, D. P., Cohen, S. N., Clark, W. G. \& Marrs, B. Alignment of genetic and restriction maps of the photosynthesis region of the Rhodopseudomonas capsulata chromosome by a conjugation-mediated marker rescue technique. J. Bact. 154, .-80-590 (1983).

Trautman, J. K., Shreve, A. P., Violette, C. A., Frank, H. A., Owens, T. G. \& Albrecht, A. C. Femtosecond dynamics of energy transfer in B800-850 lightharvesting complexes of Rhodobacter sphaeroides. Proc. Natl. Acad. Sci. 87, 215-219 (1990).

Trost, J. T., Brune, D. C. \& Blankenship, R. E. Protein sequences and redox titrations indicate that the electron acceptors in reaction centers from Heliobacteria are similar to photosystem-I. Photosynthesis Research 32, 11-22 (1992).

Van Gorkom, H. J. Photosynthesis 343-350 (1987).

Walker, C. J. \& Weinstein, J. D. In vitro assay of the chlorophyll biosynthetic enzyme $\mathrm{Mg}$-chelatase--resolution of the activity into soluble and membrane bound fractions. Proc. Natl. Acad. Sci. 88, $5789-5793$ (1991).

Wang, W.-Y. Photoconversion of protochlorophyllide in the $y-1$ mutant of Chlamydomonas reinhardtii. Plant Physiol. 63, 1102-1106 (1979). 
Warren, M. J. \& Scott, A. I. Tetrapyrrole assembly and modification into the ligands of biologically functional cofactors. Trends in Biochemical Sciences 15, 486-491 (1990).

Wastyn, M., Achatz, A., Molitor, V. \& Peschek, G. A. Respiratory activities and aa3-type cytochrome oxidase in plasma and thylakoid membranes from vegetative cells and heterocysts of the cyanobacterium Anabaena ATCC 29413. Biochim. Biophys. Acta 935, 217-224 (1988).

Weaver, P. F., Wail, J. D. \& Gest, H. Characterization of Rhodopseudomonas capsulata. Arch. Microbiol 105, 207-216 (1975).

Wellington, C. L. \& Beatty, J. T. Promoter mapping and nucleotide sequence of the bchC bacteriochlorophyll biosynthesis gene from Rhodobacter capsulatus. Gene 83, 251-261 (1989).

Wellington, C. L. \& Beatty, J. T. Overlapping mRNA transcripts of photosynthesis gene operons in Rhodobacter capsulatus. J. Bact. 173, 1432-1443 (1991).

Wierenga, R. K., Terpstra, P. \& Hol, W. G. J. Prediction of the occurrence of the ADP-binding $\beta \alpha \beta$-fold in proteins, using an amino acid sequence fingerprint. J. Mol. Biol. 187, 101-107 (1986).

Wiessner, C., Dunger, I. \& Michel; H. Structure and transcription of the genes encoding the B1015 light-harvesting complex $\beta$ and $\alpha$ subunits and the 
photosynthetic reaction center $L, M$, and cytochrome c subunits from Rhodopseudomonas viridis. J. Bact. 172, $2877-2887$ (1990).

Willets, N. \& Skurray, R. Structure and function of the F factor and mechanism of conjugation. Escherichia coli and Salmonella typhimurium cellular and molecular biology 2, 1110-1133 (1987).

Willing, A. \& Howard, J. B. Cross-linking site in Azotobacter vinelandii complex. J. Biol. Chem. 265, 6596-6599 (1990).

Willing, A. H., Georgiadia, M. M., Rees, D. C. \& Howard, J. B. Cross-linking of nitrognase components. Structure and activity of the covalent complex. J. Biol. Chem. 264, 8499-8503 (1989).

Winkenbach, F. \& Wolk, C. P. Activities of enzymes of the oxidative and the reductive pentose phosphate pathways in heterocysts of a blue-green alga. Plant Physiol. 52, 480-484 (1973).

Woese, C. R. Bacterial Evolution. Microbiol. Rev. 51, 221-271 (1987).

Woese, C. R., Debrunner-Vossbrinck, B. A., Oyaizu, H., Stackebrandt, E. \& Ludwig, W. Gram-positive bacteria: Possible photosynthetic ancestry. Science 229, $762-765$ (1985).

Woese, C. R., Kandler, O. \& Wheelis, M. L. Towards a natural system of organisms: proposal for the domains Archaea, Bacteria, and Eucarya. Proc. Natl. Acad. Sci. 87, 4576-4579 (1990). 
Wolle, D., Kim, C., Dean, D. \& Howard, J. Ionic interactions in the nitrogenase complex. Properties of Fe-protein containing substitutions for Arg-100. 262, 3667-3673 (1992).

Yang, Z. \& Bauer, C. E. Rhodobacter capsulatus genes involved in early steps of the bacteriochlorophyll biosynthesis pathway. J. Bact. 172, 5001-5010 (1990).

Yates, M. G. \& Jones, C. W. Respiration and nitrogen-fixation in Azotobacter. Adv. Microb. Physiol. 11, 97-135 (1974).

Yen, H.-c. \& Marrs, B. Map of genes for carotenoid and bacteriochlorophyll biosynthesis in Rhodopseudomonas capsulata. J. Bact. 126, 619-629 (1976).

Yen, H.-C. \& Marrs, B. Growth of Rhodopseudomonas capsulata under anaerobic dark conditions with dimethyl sulfoxide. Arch. Biochemistry and Biophysics $181,411-418$ (1977).

Yildiz, F. H., Gest, H. \& Bauer, C. E. The photosynthesis gene cluster is conserved in Rhodospirillum centenum. Mol. Microbiol. (in press).

Young, D. A., Bauer, C. E., Williams, J. C. \& Marrs, B. L. Genetic evidence for superoperonal organization of genes for photosynthetic pigments and pigment-binding proteins in Rhodobacter capsulatus. Mol. Gen. Genet. 218, 1 12 (1989). 
Youvan, D. C., Bylina, E. J., Alberti, M., Begush, H. \& Hearst, J. E. Nucleotide and deduced polypeptide sequences of the photosynthetic reaction-center, B870 antenna, and flanking polypeptides from R. capsulata. Cell 37, 949-957 (1984).

Zhu, Y. S., Cook, D. N., Leach, F., Armstrong, G. A., Alberti, M. \& Hearst, J. E. Oxygen-regulated mRNAs for light-harvesting and reaction center complexes and for bacteriochlorophyll and carotenoid biosynthesis in Rhodobacter capsulatus during the shift from anaerobic to aerobic growth. J. Bact. 168, 1180-1188 (1986).

Zsebo, K. M. Genetic-physical mapping of a photosynthetic gene cluster in Rhodopseudomonas capsulata. Ph. D. thesis. University of California, Berkeley (1984).

Zsebo, K. M. \& Hearst, J. E. Genetic-physical mapping of a photosynthetic gene cluster from $R$. capsulatus. Cell 37, $937-947$ (1984). 

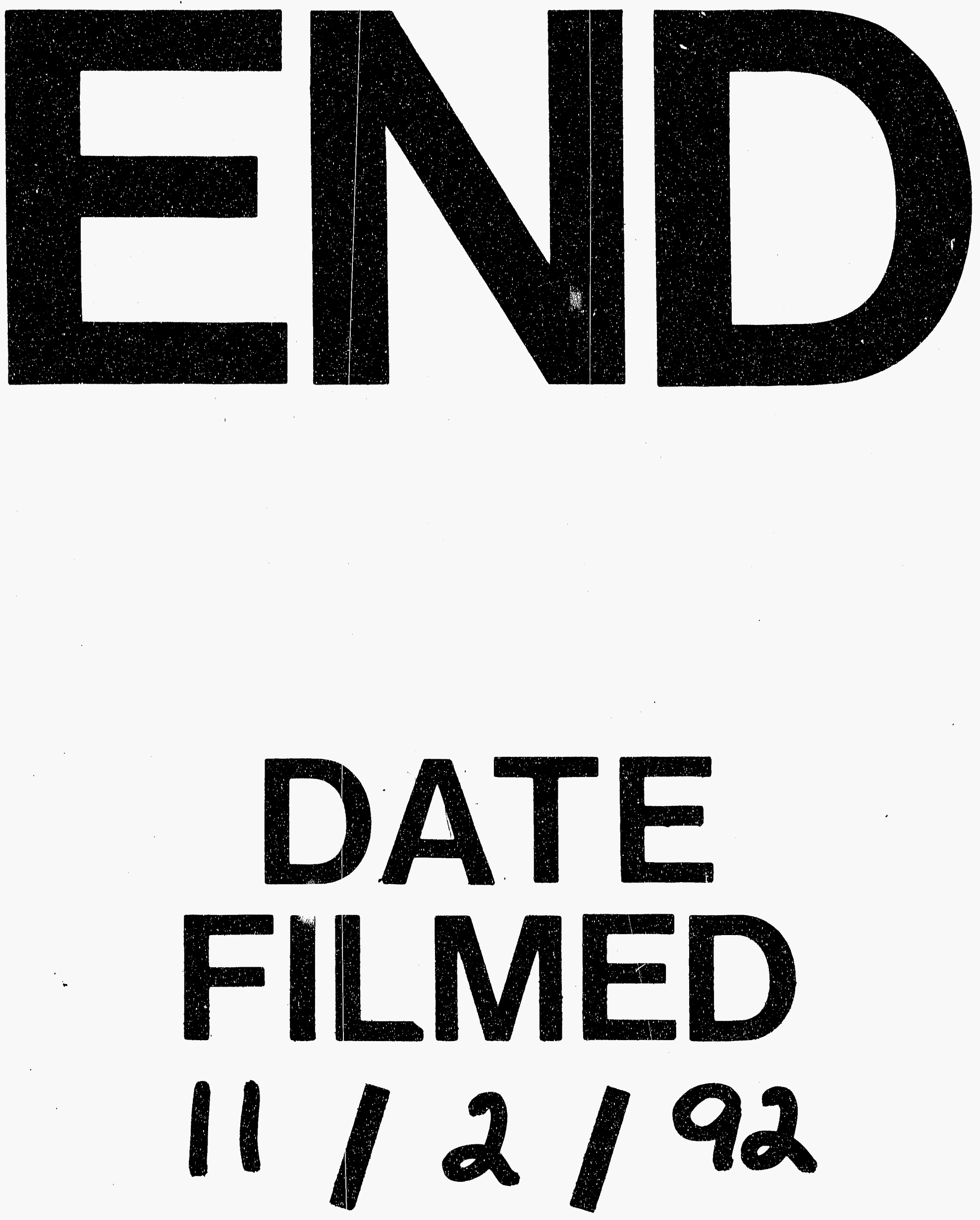
